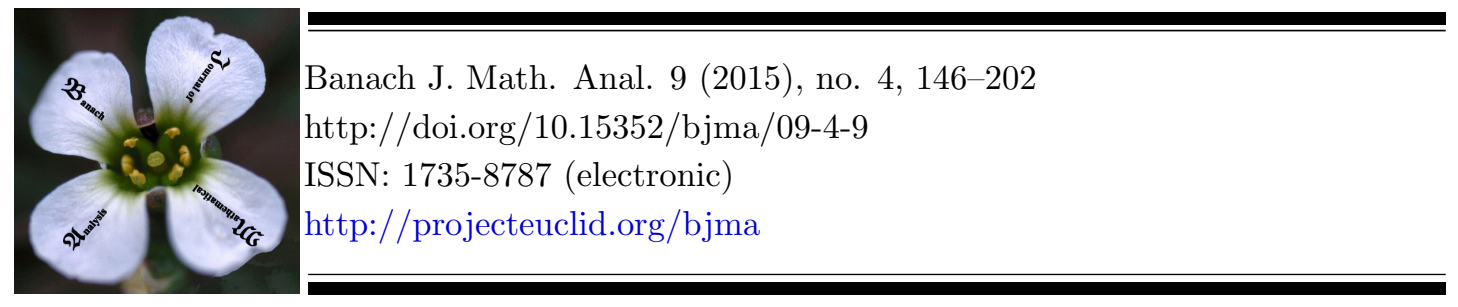

\title{
TRIEBEL-LIZORKIN TYPE SPACES WITH VARIABLE EXPONENTS
}

\author{
DACHUN YANG, CIQIANG ZHUO AND WEN YUAN*
}

Communicated by M. A. Ragusa

\begin{abstract}
In this article, the authors first introduce the Triebel-Lizorkintype space $F_{p(\cdot), q(\cdot)}^{s(\cdot)}\left(\mathbb{R}^{n}\right)$ with variable exponents, and establish its $\varphi$-transform characterization in the sense of Frazier and Jawerth, which further implies that this new scale of function spaces is well defined. The smooth molecular and the smooth atomic characterizations of $F_{p(\cdot), q(\cdot)}^{s(\cdot),}\left(\mathbb{R}^{n}\right)$ are also obtained, which are used to prove a trace theorem of $F_{p(\cdot), q(\cdot)}^{s(\cdot),}\left(\mathbb{R}^{n}\right)$. The authors also characterize the space $F_{p(\cdot), q(\cdot)}^{s(\cdot),}\left(\mathbb{R}^{n}\right)$ via Peetre maximal functions.
\end{abstract}

\section{INTRODUCTION}

Between 1960's and 1970's, the Besov space $B_{p, q}^{s}\left(\mathbb{R}^{n}\right)$ and the Triebel-Lizorkin space $F_{p, q}^{s}\left(\mathbb{R}^{n}\right)$ were introduced and investigated accompanying with the development of the theory of function spaces (see, for example, [66]). These spaces form a very general unifying scale of many well-known classical concrete function spaces such as Lebesgue spaces, Hölder-Zygmund spaces, Sobolev spaces, Besselpotential spaces, Hardy spaces and BMO, which have their own history. A comprehensive treatment of these function spaces and their history can be founded in Triebel's monographes $[66,67,68,69]$. Recently, to clarify the relations among Besov spaces, Triebel-Lizorkin spaces and $Q$ spaces (see [16, 24]), Besov-type spaces $B_{p, q}^{s, \tau}\left(\mathbb{R}^{n}\right)$ and Triebel-Lizorkin-type spaces $F_{p, q}^{s, \tau}\left(\mathbb{R}^{n}\right)$ and their homogeneous counterparts for all admissible parameters were introduced and studied in

Date: Received: Sep. 21, 2014; Accepted: Jan. 4, 2015.

* Corresponding author.

2010 Mathematics Subject Classification. Primary 46E35; Secondary 42B25, 42B35.

Key words and phrases. Triebel-Lizorkin space, variable exponent, molecule, atom, trace. 
[76, 77, 80]. Moreover, the Besov-type and the Triebel-Lizorkin-type spaces, including some of their special cases related to $Q$ spaces, have been used to study the existence and the regularity of solutions of some partial differential equations such as (fractional) Navier-Stokes equations; see, for example, [43, 44, 45, 70, 81]. For more properties of these spaces, we refer the reader to [63, 64, 78, 79].

On the other hand, in recent years, there has been a growing interesting in generalizing classical spaces such as Lebesgue and Sobolev spaces to cases with either variable integrability or variable smoothness (see $[13,19])$, which are obviously not covered by any function space with invariable exponents. Spaces of variable integrability can be traced back to Birnbaum-Orlicz [10], Orlicz [58] and Nakano [54, 55]. In particular, the definition of so-called Musielak-Orlicz spaces was clearly written by Nakano in [54, Section 89], while it seems that Orlicz was mainly interested in the completeness of function spaces. But the modern development was started with the article [37] of Kováčik and Rákosník in 1991 and widely used in the study of harmonic analysis as well as partial differential equations; see, for example, [6, 12, 13, 14, 17, 18, 19, 21, 32, 48]. The motivation to study such function spaces also comes from applications to fluid dynamic, image processing and the calculus of variation; see, for example, $[1,2,3,11,21,25,59,60]$.

To complete the theory of the variable exponent Lebesgue and Sobolev spaces, Almeida and Samko [5] and Gurka et al. [30] introduced and investigated variable exponent Bessel potential spaces $\mathcal{L}^{\alpha, p(\cdot)}$ with variable integrability index $p(\cdot)$. Later, Xu $[73,74,75]$ studied Besov spaces $B_{p(\cdot), q}^{s}\left(\mathbb{R}^{n}\right)$ and Triebel-Lizorkin spaces $F_{p(\cdot), q}^{s}\left(\mathbb{R}^{n}\right)$ with the variable exponent $p(\cdot)$ but invariable exponents $q$ and $s$. Along a different line of study, when Leopold [38, 39, 40, 41] and Leopold and Schrohe [42] studied pseudo-differential operators with symbols of the type

$$
\left(1+|\xi|^{2}\right)^{s(x) / 2}
$$

they defined and investigated related Besov spaces with variable smoothness, $B_{p, p}^{s(\cdot)}\left(\mathbb{R}^{n}\right)$. Function spaces of variable smoothness including Besov space $B_{p, q}^{s(\cdot)}\left(\mathbb{R}^{n}\right)$ and Triebel-Lizorkin space $F_{p, q}^{s(\cdot)}\left(\mathbb{R}^{n}\right)$ have been studied by Besov [7, 8, 9], which was a generalization of Leopold's work. Another interesting research direction of function spaces with variable integrability is the theory of Hardy spaces $H^{p(\cdot)}\left(\mathbb{R}^{n}\right)$ with variable exponents as well as local Hardy spaces $h^{p(\cdot)}\left(\mathbb{R}^{n}\right)$, which was introduced and investigated by Nakai and Sawano [53] and they proved that

$$
h^{p(\cdot)}\left(\mathbb{R}^{n}\right)=F_{p(\cdot), 2}^{0}\left(\mathbb{R}^{n}\right) .
$$

Independently, Cruz-Uribe and Wang in [15] also investigated the variable exponent Hardy space with some weaker conditions than those used in [53].

As we can see from the trace and the embedding theorems of the classical function spaces, the smoothness and the integrability often interact each other; see, for example, [80, Theorem 6.8 and Corollary 2.2]. As was pointed out in [4, p. 1629] and [20, p. 1733], the unifications of the trace and the Sobolev embedding do not occur on function spaces with only one index variable. For example, the trace space of the variable exponent Sobolev space $W^{k, p(\cdot)}$ is no longer a space 
of the same type (see [19]), since they involve an interaction between integrability and smoothness. As one of motivations, to tackle this problem, Alexandre and Hästö [20] introduced and investigated Triebel-Lizorkin spaces with variable smoothness and integrability $F_{p(\cdot), q(\cdot)}^{s(\cdot)}\left(\mathbb{R}^{n}\right)$ with $s(\cdot) \geq 0$, and showed that these spaces behaved nicely with respect to the trace operator. Subsequently, Vybíral [72] established Sobolev and Jawerth embeddings of these spaces and, moreover, Kempka [34] characterized $F_{p(\cdot), q(\cdot)}^{s(\cdot)}\left(\mathbb{R}^{n}\right)$ by local means, and Kempka and Vybíral [36] obtained the equivalent characterization via ball means of differences. The main difficulty of studying $F_{p(\cdot), q(\cdot)}^{s(\cdot)}\left(\mathbb{R}^{n}\right)$ is the absence of the vector-valued inequality for the boundedness on $L^{p(\cdot)}\left(\ell^{q(\cdot)}\left(\mathbb{R}^{n}\right)\right)$ of the Hardy-Littlewood maximal function, which, in the classical case with $p, q, s$ being constant exponents, is a very important tool in studying the space $F_{p, q}^{s}\left(\mathbb{R}^{n}\right)$. The vector-valued convolution inequality developed in [20, Theorem 3.2] (see also Lemma 2.9 below) supplies a well remedy for this absence.

Vybíral [72] and Kempka [34] also studied the Besov space $B_{p(\cdot), q}^{s(\cdot)}\left(\mathbb{R}^{n}\right)$ with the only index $q$ being a constant, which is a quite natural case, since the norm in the Besov space is usually defined via the iterated space $\ell^{q}\left(L^{p}\left(\mathbb{R}^{n}\right)\right)$. Furthermore, Almeida and Hästö [4] introduced and investigated the Besov space $B_{p(\cdot), q(\cdot)}^{s(\cdot)}\left(\mathbb{R}^{n}\right)$ with all three variable exponents, which makes a further step in completing the unification process of function spaces with variable smoothness and integrability. The atomic characterization of $B_{p(\cdot), q(\cdot)}^{s(\cdot)}\left(\mathbb{R}^{n}\right)$ was established by Drihem [22] and some equivalent characterizations via local means and ball means of differences were also obtained by Kempka and Vybŕal [36]. Moreover, Noi and Sawano [57] investigated the complex interpolation of Besov spaces and Triebel-Lizorkin spaces with variable exponents (see also [79] for the complex interpolation of Besov-type spaces and Triebel-Lizorkin-type spaces but with invariable exponents) and, in [56], Noi studied the trace and the extension operators for Beosv spaces and Triebel-Lizorkin spaces with variable exponents. Very recently, Izuki et al. [33] gave out an elementary introduction to function spaces with variable exponents and a survey of related function spaces.

More generally, Kempka [35] introduced and studied 2-microlocal Besov and Triebel-Lizorkin spaces with variable integrability and gave out characterizations by decompositions in atoms, molecules and wavelets, which cover the usual Besov and Triebel-Lizorkin spaces as well as spaces of variable smoothness and integrability and also include the space $F_{p(\cdot), q(\cdot)}^{s(\cdot)}\left(\mathbb{R}^{n}\right)$ without the restriction $s(\cdot) \geq 0$. The trace of 2-microlocal Besov and Triebel-Lizorkin spaces with variable exponents was studied by Moura et al. [49], as well as Gonçalves et al. [29]. Moreover, Ho [31] investigated the variable Triebel-Lizorkin-Morrey space, which is an extension of Triebel-Lizorkin-Morrey spaces in $[61,62]$ and also generalizes the function space $F_{p(\cdot), q(\cdot)}^{s(\cdot)}\left(\mathbb{R}^{n}\right)$ in $[20]$.

Here, we should point out that, different from the classical case with exponents being constants, the definition of $B_{p(\cdot), q(\cdot)}^{s(\cdot)}\left(\mathbb{R}^{n}\right)$ is more complicated than that of 
$F_{p(\cdot), q(\cdot)}^{s(\cdot)}\left(\mathbb{R}^{n}\right)$. The main reason is that the mixed Lebesgue-sequence space

$$
\ell^{q(\cdot)}\left(L^{p(\cdot)}\left(\mathbb{R}^{n}\right)\right)
$$

(see [4, Definition 3.1]) involved in the definition of $B_{p(\cdot), q(\cdot)}^{s(\cdot)}\left(\mathbb{R}^{n}\right)$, as was pointed out by Almeida and Hästö in [4, Remark 4.2], does not enjoy one key feature of iterated function spaces, namely, inheritance of properties from constituent spaces. To limit the length of this article, we leave the study of Besov-type spaces with all variable exponents in a furthercoming article.

The purpose of this article is to introduce and study a more generalized scale of function spaces, based on the Triebel-Lizorkin-type space $F_{p, q}^{s, \tau}\left(\mathbb{R}^{n}\right)$, with variable exponent of smoothness, $s(\cdot)$, variable exponents of integrability, $p(\cdot)$ and $q(\cdot)$, and a set function $\phi$, denoted by $F_{p(\cdot), q(\cdot)}^{s(\cdot),}\left(\mathbb{R}^{n}\right)$. These spaces generalize classical Triebel-Lizorkin-type spaces and Triebel-Lizorkin spaces with variable smoothness and integrability. Molecular and atomic characterizations, Peetre maximal function characterizations of these spaces are also established in this article. As applications, we show a trace theorem of Triebel-Lizorkin-type spaces with variable exponents and give out some equivalent quasi-norms under some restrictions of the set function $\phi$.

We begin with some basic notation. In what follows, for a measurable function $p(\cdot): \mathbb{R}^{n} \rightarrow(0, \infty)$ and a measurable set $E$ of $\mathbb{R}^{n}$, let

$$
p_{-}(E):=\underset{x \in E}{\operatorname{essinf}} p(x) \text { and } p_{+}(E):=\underset{x \in E}{\operatorname{ess} \sup } p(x) .
$$

For notational simplicity, we let $p_{-}:=p_{-}\left(\mathbb{R}^{n}\right)$ and $p_{+}:=p_{+}\left(\mathbb{R}^{n}\right)$. Denote by $\mathcal{P}\left(\mathbb{R}^{n}\right)$ the collection of all measurable functions $p(\cdot): \mathbb{R}^{n} \rightarrow(0, \infty)$ satisfying $0<p_{-} \leq p_{+}<\infty$.

For $p(\cdot) \in \mathcal{P}\left(\mathbb{R}^{n}\right)$ and a measurable set $E \subset \mathbb{R}^{n}$, the space $L^{p(\cdot)}(E)$ is defined to be the set of all measurable functions $f$ such that

$$
\|f\|_{L^{p(\cdot)}(E)}:=\inf \left\{\lambda \in(0, \infty): \int_{E}\left[\frac{|f(x)|}{\lambda}\right]^{p(x)} d x \leq 1\right\}<\infty .
$$

For $r \in(0, \infty)$, denote by $L_{\text {loc }}^{r}\left(\mathbb{R}^{n}\right)$ the set of all $r$-locally integrable functions on $\mathbb{R}^{n}$. Denote by $L^{\infty}\left(\mathbb{R}^{n}\right)$ the set of all measurable functions $f$ such that

$$
\|f\|_{L^{\infty}\left(\mathbb{R}^{n}\right)}:=\operatorname{essips}_{y \in \mathbb{R}^{n}}|f(y)|<\infty .
$$

Remark 1.1. Let $p(\cdot) \in \mathcal{P}\left(\mathbb{R}^{n}\right)$.

(i) It was presented in [53, p. 3671] (see also [13, Theorem 2.17]) that, for all $\lambda \in \mathbb{C}$,

$$
\|\lambda f\|_{L^{p(\cdot)\left(\mathbb{R}^{n}\right)}}=|\lambda|\|f\|_{L^{p(\cdot)}\left(\mathbb{R}^{n}\right)}
$$

and, if $r \in\left(0, \min \left\{p_{-}, 1\right\}\right]$, then, for all $f, g \in L^{p(\cdot)}\left(\mathbb{R}^{n}\right)$,

$$
\|f+g\|_{L^{p(\cdot)}\left(\mathbb{R}^{n}\right)}^{r} \leq\|f\|_{L^{p(\cdot)}\left(\mathbb{R}^{n}\right)}^{r}+\|g\|_{L^{p(\cdot)}\left(\mathbb{R}^{n}\right)}^{r} .
$$

(ii) If

$$
\int_{\mathbb{R}^{n}}\left[\frac{|f(x)|}{\delta}\right]^{p(x)} d x \leq \widetilde{C}
$$


for some $\delta \in(0, \infty)$ and some positive constant $\widetilde{C}$ independent of $\delta$, then it is easy to see that $\|f\|_{L^{p(\cdot)}\left(\mathbb{R}^{n}\right)} \leq C \delta$, where $C$ is a positive constant independent of $\delta$, but depending on $p_{-}$(or $\left.p_{+}\right)$and $\widetilde{C}$.

(iii) Let $p(\cdot) \in \mathcal{P}\left(\mathbb{R}^{n}\right)$ satisfy $1<p_{-} \leq p_{+}<\infty$. Define the conjugate exponent $\widetilde{p}(\cdot)$ of $p(\cdot)$ by setting, for all $x \in \mathbb{R}^{n}, \widetilde{p}(x):=\frac{p(x)}{p(x)-1}$. It was proved, in [13, Theorem 2.6], that, if $f \in L^{p(\cdot)}\left(\mathbb{R}^{n}\right)$ and $g \in L^{\widetilde{p}(\cdot)}\left(\mathbb{R}^{n}\right)$, then $f g \in L^{1}\left(\mathbb{R}^{n}\right)$ and

$$
\int_{\mathbb{R}^{n}}|f(x) g(x)| d x \leq C\|f\|_{L^{p(\cdot)}\left(\mathbb{R}^{n}\right)}\|g\|_{L^{\tilde{p}(\cdot)}\left(\mathbb{R}^{n}\right)},
$$

where $C$ is a positive constant depending on $p_{-}$or $p_{+}$, but independent of $f$ and $g$. then

(iv) Obviously, the space $L^{p(\cdot)}\left(\mathbb{R}^{n}\right)$ has the lattice property, namely, if $|f| \leq|g|$,

$$
\|f\|_{L^{p(\cdot)\left(\mathbb{R}^{n}\right)}} \leq\|g\|_{L^{p(\cdot)\left(\mathbb{R}^{n}\right)}} .
$$

Recall that a measurable function $g \in \mathcal{P}\left(\mathbb{R}^{n}\right)$ is said to satisfy the locally logHölder continuous condition, denoted by $g \in C_{\mathrm{loc}}^{\log }\left(\mathbb{R}^{n}\right)$, if there exists a positive constant $C_{\log }(g)$ such that, for all $x, y \in \mathbb{R}^{n}$,

$$
|g(x)-g(y)| \leq \frac{C_{\log }(g)}{\log (e+1 /|x-y|)},
$$

and $g$ is said to satisfy the globally log-Hölder continuous condition, denoted by $g \in C^{\log }\left(\mathbb{R}^{n}\right)$, if $g \in C_{\mathrm{loc}}^{\log }\left(\mathbb{R}^{n}\right)$ and there exist positive constants $C_{\infty}$ and $g_{\infty}$ such that, for all $x \in \mathbb{R}^{n}$,

$$
\left|g(x)-g_{\infty}\right| \leq \frac{C_{\infty}}{\log (e+|x|)}
$$

Remark 1.2. (i) Let $g \in C^{\log }\left(\mathbb{R}^{n}\right)$. Then $g_{\infty}=\lim _{|x| \rightarrow \infty} p(x)$.

(ii) Let $g \in \mathcal{P}\left(\mathbb{R}^{n}\right)$. Then $g \in C^{\log }\left(\mathbb{R}^{n}\right)$ if and only if $1 / g \in C^{\log }\left(\mathbb{R}^{n}\right)$.

For all $x \in \mathbb{R}^{n}$ and $r \in(0, \infty)$, denote by $Q(x, r)$ the cube centered at $x$ with side-length $r$, whose sides parallel axes of coordinates. Let $\phi: \mathbb{R}^{n} \times[0, \infty) \rightarrow$ $(0, \infty)$ be a measurable function. In this article, we always suppose that $\phi$ satisfies the following two conditions:

(S1) there exist positive constants $c_{1}$ and $\widetilde{c}_{1}$ such that, for all $x \in \mathbb{R}^{n}$ and $r \in(0, \infty)$,

$$
\left(\widetilde{c}_{1}\right)^{-1} \leq \frac{\phi(x, r)}{\phi(x, 2 r)} \leq c_{1}
$$

(S2) there exists a positive constant $c_{2}$ such that, for all $x, y \in \mathbb{R}^{n}$ and $r \in$ $(0, \infty)$ with $|x-y| \leq r$,

$$
\left(c_{2}\right)^{-1} \leq \frac{\phi(x, r)}{\phi(y, r)} \leq c_{2} .
$$

In what follows, for all cubes $Q:=Q(x, r)$ with $x \in \mathbb{R}^{n}$ and $r \in(0, \infty)$, let

$$
\phi(Q):=\phi(Q(x, r)):=\phi(x, r) .
$$


Remark 1.3. (i) We point out that the conditions $(\mathbf{S 1})$ and $(\mathbf{S 2})$ of $\phi$ are, respectively, called the doubling condition and the compatibility condition, which have been used by Nakai [50, 51] and Nakai and Sawano [53] when they studied generalized Campanato spaces.

(ii) Let $\phi(Q):=|Q|^{\tau}$ with $\tau \in[0, \infty)$ for all cubes $Q$. Then, obviously, $\phi$ satisfies the conditions $(\mathbf{S 1})$ and $(\mathbf{S 2})$.

(iii) Let $p(\cdot) \in C^{\log }\left(\mathbb{R}^{n}\right)$. The set function $\phi$, defined by setting, for all cubes Q,

$$
\phi(Q):=\frac{\left\|\chi_{Q}\right\|_{L^{p(\cdot)\left(\mathbb{R}^{n}\right)}}}{|Q|}
$$

which is just [53, Example 6.4], satisfies the conditions (S1) and (S2).

(iv) Let $\phi$ be a nondecreasing set function, namely, there exists a positive constant $C$ such that, for all cubes $Q_{1} \subset Q_{2}, \phi\left(Q_{1}\right) \leq C \phi\left(Q_{2}\right)$. If $\phi$ satisfies the condition (S1), then $\phi$ also satisfies the condition (S2). Indeed, for all $x, y \in \mathbb{R}^{n}$ and $r \in(0, \infty)$ with $|x-y| \leq r$, it is easy to see that $Q(x, r) \subset Q(y, 2 r)$ and $Q(y, r) \subset Q(x, 2 r)$ and, by the condition $(\mathbf{S} 1)$, we see that

$$
1 \lesssim \frac{\phi(x, r)}{\phi(x, 2 r)} \lesssim \frac{\phi(x, r)}{\phi(y, r)} \lesssim \frac{\phi(y, 2 r)}{\phi(y, r)} \lesssim 1
$$

with the implicit positive constants independent of $x, y$ and $r$. Thus, $\phi$ satisfies the condition (S2).

(v) Let $\phi(Q):=\int_{Q} w(x) d x$ for all cubes $Q$, where $w$ is a classical Muckenhoupt $A_{p}\left(\mathbb{R}^{n}\right)$-weight with $p \in[1, \infty]$. It is well known that each Muckenhoupt $A_{p}\left(\mathbb{R}^{n}\right)$ weight is doubling, thus, by (iv), we conclude that $\phi$ satisfies the conditions (S1) and $(\mathbf{S 2})$. For the definition and properties of Muckenhoupt $A_{p}\left(\mathbb{R}^{n}\right)$-weights, we refer the reader to [65].

Let $\mathcal{S}\left(\mathbb{R}^{n}\right)$ be the space of all Schwartz functions on $\mathbb{R}^{n}$ and $\mathcal{S}^{\prime}\left(\mathbb{R}^{n}\right)$ its topological dual space. We say a pair $(\varphi, \Phi)$ of functions to be admissible if $\varphi, \Phi \in \mathcal{S}\left(\mathbb{R}^{n}\right)$ satisfy

$$
\operatorname{supp} \widehat{\varphi} \subset\left\{\xi \in \mathbb{R}^{n}: \frac{1}{2} \leq|\xi| \leq 2\right\} \text { and }|\widehat{\varphi}(\xi)| \geq c>0 \text { when } \frac{3}{5} \leq|\xi| \leq \frac{5}{3}
$$

and

$$
\operatorname{supp} \widehat{\Phi} \subset\left\{\xi \in \mathbb{R}^{n}:|\xi| \leq 2\right\} \text { and }|\widehat{\Phi}(\xi)| \geq c>0 \text { when }|\xi| \leq \frac{5}{3},
$$

where $\widehat{f}(\xi):=\int_{\mathbb{R}^{n}} f(x) e^{-i x \cdot \xi} d x$ for all $\xi \in \mathbb{R}^{n}$ and $f \in L^{1}\left(\mathbb{R}^{n}\right)$, and $c$ is a positive

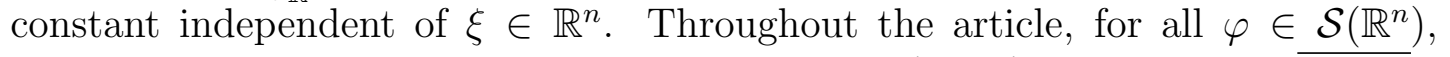
$j \in \mathbb{N}:=\{1,2, \ldots\}$ and $x \in \mathbb{R}^{n}$, we put $\varphi_{j}(x):=2^{j n} \varphi\left(2^{j} x\right)$ and $\widetilde{\varphi}(x):=\overline{\varphi(-x)}$.

For $j \in \mathbb{Z}$ and $k \in \mathbb{Z}^{n}$, denote by $Q_{j k}$ the dyadic cube $2^{-j}\left([0,1)^{n}+k\right), x_{Q_{j k}}:=$ $2^{-j} k$ its lower left corner and $\ell\left(Q_{j k}\right)$ its side length. Let

$$
\mathcal{Q}:=\left\{Q_{j k}: j \in \mathbb{Z}, k \in \mathbb{Z}^{n}\right\}, \mathcal{Q}^{*}:=\{Q \in \mathcal{Q}: \ell(Q) \leq 1\}
$$

and $j_{Q}:=-\log _{2} \ell(Q)$ for all $Q \in \mathcal{Q}$.

Now we introduce Triebel-Lizorkin-type spaces with variable exponents. 
Definition 1.4. Let $(\varphi, \Phi)$ be a pair of admissible functions on $\mathbb{R}^{n}$. Let $p, q \in$ $\mathcal{P}\left(\mathbb{R}^{n}\right)$ satisfy

$$
0<p_{-} \leq p_{+}<\infty, 0<q_{-} \leq q_{+}<\infty
$$

and $\frac{1}{p}, \frac{1}{q} \in C^{\log }\left(\mathbb{R}^{n}\right), s \in C_{\operatorname{loc}}^{\log }\left(\mathbb{R}^{n}\right) \cap L^{\infty}\left(\mathbb{R}^{n}\right)$ and $\phi$ be a set function satisfying the conditions $(\mathbf{S 1})$ and $(\mathbf{S 2})$. Then the Triebel-Lizorkin-type space with variable exponents, $F_{p(\cdot), q(\cdot)}^{s(\cdot),}\left(\mathbb{R}^{n}\right)$, is defined to be the set of all $f \in \mathcal{S}^{\prime}\left(\mathbb{R}^{n}\right)$ such that

$$
\|f\|_{F_{p(\cdot), q(\cdot)}^{s(\cdot),\left(\mathbb{R}^{n}\right)}}:=\sup _{P \in \mathcal{Q}} \frac{1}{\phi(P)}\left\|\left\{\sum_{j=\max \left\{j_{P}, 0\right\}}^{\infty}\left[2^{j s(\cdot)}\left|\varphi_{j} * f(\cdot)\right|\right]^{q(\cdot)}\right\}^{\frac{1}{q(\cdot)}}\right\|_{L^{p(\cdot)}(P)}<\infty,
$$

where, when $j=0, \varphi_{0}$ is replaced by $\Phi$, and the supremum is taken over all dyadic cubes $P$ in $\mathbb{R}^{n}$.

Remark 1.5. Let $p(\cdot), q(\cdot), s(\cdot)$ be as in Definition 1.4.

(i) When $\phi(Q):=1$ for all cubes $Q$, then

$$
F_{p(\cdot), q(\cdot)}^{s(\cdot)}\left(\mathbb{R}^{n}\right)=F_{p(\cdot), q(\cdot)}^{s(\cdot)}\left(\mathbb{R}^{n}\right)
$$

where $F_{p(\cdot), q(\cdot)}^{s(\cdot)}\left(\mathbb{R}^{n}\right)$ denotes the Triebel-Lizorkin space with variable smoothness and integrability which is introduced and investigated in [20]. We point out that Diening et al. [20] studied the space $F_{p(\cdot), q(\cdot)}^{s(\cdot)}\left(\mathbb{R}^{n}\right)$ under an additional assumption that $s$ is nonnegative, which is generalized to the case that $s: \mathbb{R}^{n} \rightarrow \mathbb{R}$ and $s \in C_{\text {loc }}^{\log }\left(\mathbb{R}^{n}\right) \cap L^{\infty}\left(\mathbb{R}^{n}\right)$ by Kempka in [35].

(ii) When $p, q, s$ are constant exponents and $\phi(Q):=|Q|^{\tau}$ with $\tau \in[0, \infty)$ for all cubes $Q$, then

$$
F_{p(\cdot), q(\cdot)}^{s(\cdot),}\left(\mathbb{R}^{n}\right)=F_{p, q}^{s, \tau}\left(\mathbb{R}^{n}\right),
$$

where $F_{p, q}^{s, \tau}\left(\mathbb{R}^{n}\right)$ denotes the Triebel-Lizorkin-type space which was introduced and studied in [80].

(iii) When $q, s$ are constant exponents and $\phi(Q):=|Q|^{\tau}$ with $\tau \in[0, \infty)$ for all cubes $Q$, then

$$
F_{p(\cdot), q(\cdot)}^{s(\cdot),}\left(\mathbb{R}^{n}\right)=F_{p(\cdot), q}^{s, \tau}\left(\mathbb{R}^{n}\right),
$$

which was investigated in [47].

(iv) The condition, $0<p_{-} \leq p_{+}<\infty$, is quite natural, since there also exists the restriction $p<\infty$ in the case of constant exponents. The assumption, $0<q_{-} \leq q_{+}<\infty$, is different from the case of constant exponents where $q=\infty$ is included. This restriction comes from the application of the convolution inequality in [20, Theorem 3.2] (see also Lemma 2.9 below), when proving that the space $F_{p(\cdot), q(\cdot)}^{s(\cdot),}\left(\mathbb{R}^{n}\right)$ is independent of the choice of admissible function pairs $(\varphi, \Phi)$. Observe that, even when $\phi(Q)=1$ for all cubes $Q$, this restriction is necessary; see [36, p. 857].

This article is organized as follows. 
Section 2 is devoted to showing that the space $F_{p(\cdot), q(\cdot)}^{s(\cdot),}\left(\mathbb{R}^{n}\right)$ is independent of the choice of admissible function pairs $(\varphi, \Phi)$, which is a consequence of the $\varphi$ transform characterization of $F_{p(\cdot), q(\cdot)}^{s(\cdot),}\left(\mathbb{R}^{n}\right)$ in the sense of Frazier and Jawerth (see Corollary 2.4 below). Different from the method used in the case of constant exponents, in the proof of the boundedness of the $\varphi$-transform $S_{\varphi}$ from $F_{p(\cdot), q(\cdot)}^{s(\cdot),}\left(\mathbb{R}^{n}\right)$ to $f_{p(\cdot), q(\cdot)}^{s(\cdot),}\left(\mathbb{R}^{n}\right)$ (the sequence space corresponding to the function space $F_{p(\cdot), q(\cdot)}^{s(\cdot),}\left(\mathbb{R}^{n}\right)$ ), we make full use of the so-called $r$-trick lemma (namely, [20, Lemma A.6]) and the vector-valued convolution inequality (namely, [20, Theorem 3.2]; see also Lemma 2.9 below). We point out that the vector-valued convolution inequality also plays an essential role throughout the remainder of this article.

In Section 3, we establish equivalent characterizations of $F_{p(\cdot), q(\cdot)}^{s(\cdot),}\left(\mathbb{R}^{n}\right)$ in term of molecules, atoms (see Theorem 3.8 below) or Peetre maximal functions (see Theorem 3.11 below). To prove Theorem 3.8, we borrow some ideas from the proof of [23, Theorem 3.12] which gives the atomic characterization of the Besov-type and the Triebel-Lizorkin-type spaces, and the proof of [35, Theorem 3.13] which gives the molecular characterization of 2-microlocal Besov and Triebel-Lizorkin spaces with variable integrability. The Sobolev embedding (see Proposition 3.1 below) plays a key role in the proof of Theorem 3.8, which may be of independent interest. The proof of Theorem 3.11 is similar to that of [46, Theorem 3.2] (see also [71, Theorem 2.6]) and strongly depends on the vector-valued convolution inequality on $L^{p(\cdot)}\left(\ell^{q(\cdot)}\left(\mathbb{R}^{n}\right)\right)$; see Lemma 2.9 below. As applications of Theorem 3.11, some equivalent norms of $F_{p(\cdot), q(\cdot)}^{s(\cdot),}\left(\mathbb{R}^{n}\right)$ are obtained (see Theorem 3.12 below), which are further used to show that the spaces $F_{p(\cdot), q(\cdot)}^{s(\cdot),}\left(\mathbb{R}^{n}\right)$ include the Morrey space with variable exponents $\mathcal{M}_{\phi}^{p(\cdot)}\left(\mathbb{R}^{n}\right)$ as a special case; see Proposition 3.18 below.

At the end of Section 3, via some examples, we show that, in general, the scales of Triebel-Lizorkin-type spaces with variable exponents and variable TriebelLizorkin-Morrey spaces (see [31]) do not cover each other (see Remark 3.15 below). Here we point out that Triebel-Lizorkin-type spaces with variable exponents in this article cover the Triebel-Lizorkin-type spaces $F_{p, q}^{s, \tau}\left(\mathbb{R}^{n}\right)$ for all $\tau \in[0, \infty)$, but the variable Triebel-Lizorkin-Morrey space in [31] does only cover the TriebelLizorkin-type space $F_{p, q}^{s, \tau}\left(\mathbb{R}^{n}\right)$ for $\tau \in[0,1 / p)$.

In Section 4, as an application of the atomic characterization of $F_{p(\cdot), q(\cdot)}^{s(\cdot), \phi}\left(\mathbb{R}^{n}\right)$, we mainly establish a trace theorem of Triebel-Lizorkin-type spaces with variable exponents (see Theorem 4.1 below). In the case that $\phi$ is as in Remark 1.5(i), the corresponding result of Theorem 4.1 was obtained in [20, Theorem 3.13] with a certain weaker condition (see Remark 4.2(ii) below), however, the convergence of the trace of $f \in F_{p(\cdot), q(\cdot)}^{s(\cdot)}\left(\mathbb{R}^{n}\right)$ was not given out exactly in [20, p. 1760]. In Section 4, we first show that the trace operator is well defined on the space $F_{p(\cdot), q(\cdot)}^{s(\cdot),}\left(\mathbb{R}^{n}\right)$ (see Lemma 4.3 below), with a certain restriction on $p$ and $s$, by an argument similar to that used in the proof of Theorem 3.8(i). Indeed, in Lemma 4.3 below, we prove that the trace of $f \in F_{p(\cdot), q(\cdot)}^{s(\cdot)}\left(\mathbb{R}^{n}\right)$ converges in $\mathcal{S}^{\prime}\left(\mathbb{R}^{n-1}\right)$. Then, similar to the proof of [20, Theorem 3.13], we show that the trace space of 
$F_{p(\cdot), q(\cdot)}^{s(\cdot),}\left(\mathbb{R}^{n}\right)$ is independent of the $n$-th coordinate of variable exponents $p(\cdot)$ and $s(\cdot)$, and complete the proof by an argument similar to that used in the proof of [80, Theorem 6.8].

Finally, we make some conventions on notation. Throughout this article, we denote by $C$ a positive constant which is independent of the main parameters, but may vary from line to line. The symbols $A \lesssim B$ means $A \leq C B$. If $A \lesssim B$ and $B \lesssim A$, then we write $A \sim B$. For all $a, b \in \mathbb{R}$, let

$$
a \vee b:=\max \{a, b\}
$$

If $E$ is a subset of $\mathbb{R}^{n}$, we denote by $\chi_{E}$ its characteristic function. For all cubes $Q$, we use $c_{Q}$ to denote its the center. For all $k:=\left(k_{1}, \ldots, k_{n}\right) \in \mathbb{Z}^{n}$, let

$$
|k|:=\left|k_{1}\right|+\cdots+\left|k_{n}\right| \text {. }
$$

Let $\mathbb{N}:=\{1,2, \ldots\}$ and $\mathbb{Z}_{+}:=\{0\} \cup \mathbb{N}$.

\section{THE $\varphi$-TRANSFORM CHARACTERIZATION}

In this section, we first introduce the sequence space $f_{p(\cdot), q(\cdot)}^{s(\cdot),}\left(\mathbb{R}^{n}\right)$ corresponding to the space $F_{p(\cdot), q(\cdot)}^{s(\cdot),}\left(\mathbb{R}^{n}\right)$ and then establish their $\varphi$-transform characterization in the sense of Frazier and Jawerth [27]. As a consequence of the $\varphi$-transform characterization, we conclude that the space $F_{p(\cdot), q(\cdot)}^{s(\cdot), \phi}\left(\mathbb{R}^{n}\right)$ is independent of the choice of admissible function pairs $(\varphi, \Phi)$.

Definition 2.1. Let $p(\cdot), s(\cdot)$ and $\phi$ be as in Definition 1.4 and $q(\cdot)$ as either in Definition 1.4 or $q(\cdot) \equiv \infty$. Then the sequence space $f_{p(\cdot), q(\cdot)}^{s(\cdot),}\left(\mathbb{R}^{n}\right)$ is defined to be the set of all sequences $t:=\left\{t_{Q}\right\}_{Q \in \mathcal{Q}^{*}} \subset \mathbb{C}$ such that

$$
\|t\|_{f_{p(\cdot), q(\cdot)}^{s(\cdot) \phi}\left(\mathbb{R}^{n}\right)}:=\sup _{P \in \mathcal{Q}} \frac{1}{\phi(P)}\left\|\left\{\sum_{Q \subset P, Q \in \mathcal{Q}^{*}}\left[|Q|^{-\left[\frac{s(\cdot)}{n}+\frac{1}{2}\right]}\left|t_{Q}\right| \chi_{Q}\right]^{q(\cdot)}\right\}^{\frac{1}{q(\cdot)}}\right\|_{L^{p(\cdot)}(P)}<\infty
$$

with the usual modification made when $q(\cdot) \equiv \infty$, where the supremum is taken over all dyadic cubes $P$ in $\mathbb{R}^{n}$.

Remark 2.2. (i) It is easy to see that $f_{p(\cdot), q(\cdot)}^{s(\cdot), \phi}\left(\mathbb{R}^{n}\right)$ is a quasi-Banach lattice, namely, for all $t^{(1)}:=\left\{t_{Q}^{(1)}\right\}_{Q \in \mathcal{Q}^{*}} \subset \mathbb{C}$ and $t^{(2)}:=\left\{t_{Q}^{(2)}\right\}_{Q \in \mathcal{Q}^{*}} \subset \mathbb{C}$, if $\left|t_{Q}^{(1)}\right| \leq\left|t_{Q}^{(2)}\right|$ for all $Q \in \mathcal{Q}^{*}$, then

$$
\left\|t^{(1)}\right\|_{f_{p(\cdot), q(\cdot)}^{s(\cdot),}\left(\mathbb{R}^{n}\right)} \leq\left\|t^{(2)}\right\|_{f_{p(\cdot), q(\cdot)}^{s(\cdot),}\left(\mathbb{R}^{n}\right)} .
$$

(ii) Let $\mathcal{D}_{0}\left(\mathbb{R}^{n}\right):=\left\{Q \subset \mathbb{R}^{n}: Q\right.$ is a cube and $\ell(Q)=2^{-j_{0}}$ for some $\left.j_{0} \in \mathbb{Z}\right\}$. Then it is easy to prove that the supremum in Definitions 1.4 and 2.1 can be equivalently taken over all cubes in $\mathcal{D}_{0}\left(\mathbb{R}^{n}\right)$, the details being omitted.

Let $(\varphi, \Phi)$ be a pair of admissible functions. Then $(\widetilde{\varphi}, \widetilde{\Phi})$ is also a pair of admissible functions. Thus, by [27, pp. 130-131] or [28, Lemma (6.9)], we know that 
there exist Schwartz functions $\psi$ and $\Psi$ satisfying (1.2) and (1.3), respectively, such that, for all $\xi \in \mathbb{R}^{n}$,

$$
\widehat{\Phi}(\xi) \widehat{\Psi}(\xi)+\sum_{j=1}^{\infty} \widehat{\varphi}\left(2^{-j} \xi\right) \widehat{\psi}\left(2^{-j} \xi\right)=1 .
$$

Recall that the $\varphi$-transform $S_{\varphi}$ is defined to be the mapping taking each $f \in$ $\mathcal{S}^{\prime}\left(\mathbb{R}^{n}\right)$ to the sequence $S_{\varphi}(f):=\left\{\left(S_{\varphi} f\right)_{Q}\right\}_{Q \in \mathcal{Q}^{*}}$, where $\left(S_{\varphi} f\right)_{Q}:=|Q|^{1 / 2} \Phi * f\left(x_{Q}\right)$ if $\ell(Q)=1$ and $\left(S_{\varphi} f\right)_{Q}:=|Q|^{1 / 2} \varphi_{j_{Q}} * f\left(x_{Q}\right)$ if $\ell(Q)<1$; the inverse $\varphi$-transform $T_{\psi}$ is defined to be the mapping taking a sequence $t:=\left\{t_{Q}\right\}_{Q \in \mathcal{Q}^{*}} \subset \mathbb{C}$ to

$$
T_{\psi} t:=\sum_{Q \in \mathcal{Q}^{*}, \ell(Q)=1} t_{Q} \Psi_{Q}+\sum_{Q \in \mathcal{Q}^{*}, \ell(Q)<1} t_{Q} \psi_{Q} ;
$$

see, for example, [80, p. 31].

Now we state the following $\varphi$-transform characterization for $F_{p(\cdot), q(\cdot)}^{s(\cdot),}\left(\mathbb{R}^{n}\right)$, which is the main result of this section. For the corresponding result of Triebel-Lizorkintype spaces, see [80, Theorem 2.1].

Theorem 2.3. Let $p, q, s$ and $\phi$ be as in Definition 1.4 and $\varphi, \psi, \Phi$ and $\Psi$ be as in (2.1). Then the operators $S_{\varphi}: F_{p(\cdot), q(\cdot)}^{s(\cdot),}\left(\mathbb{R}^{n}\right) \rightarrow f_{p(\cdot), q(\cdot)}^{s(\cdot),}\left(\mathbb{R}^{n}\right)$ and $T_{\psi}: f_{p(\cdot), q(\cdot)}^{s(\cdot),}\left(\mathbb{R}^{n}\right) \rightarrow F_{p(\cdot), q(\cdot)}^{s(\cdot), \phi}\left(\mathbb{R}^{n}\right)$ are bounded. Furthermore, $T_{\psi} \circ S_{\varphi}$ is the identity on $F_{p(\cdot), q(\cdot)}^{s(\cdot),}\left(\mathbb{R}^{n}\right)$.

We remark that $T_{\psi}$ is well defined for all $t \in f_{p(\cdot), q(\cdot)}^{s(\cdot)}\left(\mathbb{R}^{n}\right)$; see Lemma 2.5 below. The proof of Theorem 2.3 is given later. From Theorem 2.3 and an argument similar to that used in the proof of [27, Remark 2.6], we immediately deduce the following conclusion, the details being omitted.

Corollary 2.4. With all the notation as in Definition 1.4, the space $F_{p(\cdot), q(\cdot)}^{s(\cdot), \phi}\left(\mathbb{R}^{n}\right)$ is independent of the choice of the admissible function pairs $(\varphi, \Phi)$.

Now we start to show Theorem 2.3. First, we need the following property.

Lemma 2.5. Let $p, q, s$ and $\phi$ be as in Definition 1.4. Then, for all $t \in$ $f_{p(\cdot), q(\cdot)}^{s(\cdot),}\left(\mathbb{R}^{n}\right)$,

$$
T_{\psi} t:=\sum_{Q \in \mathcal{Q}^{*}, \ell(Q)=1} t_{Q} \Psi_{Q}+\sum_{Q \in \mathcal{Q}^{*}, \ell(Q)<1} t_{Q} \psi_{Q}
$$

converges in $\mathcal{S}^{\prime}\left(\mathbb{R}^{n}\right)$; moreover, $T_{\psi}: f_{p(\cdot), q(\cdot)}^{s(\cdot), \phi}\left(\mathbb{R}^{n}\right) \rightarrow \mathcal{S}^{\prime}\left(\mathbb{R}^{n}\right)$ is continuous.

To prove Lemma 2.5, we need the following technical lemmas.

Lemma 2.6. Let $\phi$ be a set function satisfying the conditions (S1) and (S2). Then

(i) there exists a positive constant $C$ such that, for any $j \in \mathbb{Z}_{+}$and $k \in \mathbb{Z}^{n}$,

$$
\phi\left(Q_{j k}\right) \leq C 2^{j \log _{2} c_{1}}(|k|+1)^{\log _{2}\left(c_{1} \widetilde{c}_{1}\right)} ;
$$


(ii) there exists a positive constant $C$ such that, for all $Q \in \mathcal{Q}$ and $l \in \mathbb{Z}^{n}$,

$$
\frac{\phi(Q+l \ell(Q))}{\phi(Q)} \leq C(1+|l|)^{\log _{2}\left(c_{1} \widetilde{c}_{1}\right)}
$$

where $c_{1}$ and $\widetilde{c}_{1}$ are as in the condition $(\boldsymbol{S} \mathbf{1})$.

Proof. We first prove (i). For any $j \in \mathbb{Z}_{+}$and $k \in \mathbb{Z}^{n}$, let $\delta_{k} \in \mathbb{Z}_{+}$be such that $2^{\delta_{k}} \leq n(|k|+1)<2^{\delta_{k}+1}$ and $c_{Q_{j k}}$ the center of the cube $Q_{j k}$. Then, by the conditions (S1) and $(\mathbf{S} 2)$ of $\phi$ and the fact that $\left|c_{Q_{j k}}\right| \leq n 2^{-j}(|k|+1)$, we see that

$$
\begin{aligned}
\phi\left(Q_{j k}\right) & =\phi\left(Q\left(c_{Q j k}, 2^{-j}\right)\right) \leq c_{1}^{\delta_{k}+1} \phi\left(Q\left(c_{Q_{j k}}, 2^{-j+\delta_{k}+1}\right)\right) \\
& \leq c_{2} c_{1}^{\delta_{k}+1} \phi\left(Q\left(0,2^{-j+\delta_{k}+1}\right)\right) \lesssim c_{1}^{j+\delta_{k}}\left(\widetilde{c}_{1}\right)^{\delta_{k}} \phi(Q(0,1)) \\
& \lesssim 2^{j \log _{2} c_{1}}(|k|+1)^{\log _{2}\left(c_{1} \widetilde{c}_{1}\right)},
\end{aligned}
$$

which completes the proof of (i).

Next we show (ii). If $|l| \leq 1$, namely, $\ell(Q)|l| \leq \ell(Q)$, then, by the condition (S2) of $\phi$, we find that

$$
c_{2}^{-1} \leq \frac{\phi(Q+l \ell(Q))}{\phi(Q)} \leq c_{2} .
$$

If $|l|>1$, namely, $\ell(Q)|l|>\ell(Q)$, then there exists a $\gamma_{l} \in \mathbb{N}$ such that $2^{\gamma_{l}} \leq|l|<$ $2^{\gamma_{l}+1}$. Thus, $\ell(Q) 2^{\gamma_{l}+1}>\ell(Q)|l|$. From the condition $(\mathbf{S} 1)$ of $\phi$, we deduce that

$$
\begin{aligned}
\phi(Q+l \ell(Q)) & =\phi\left(Q\left(c_{Q}+l \ell(Q), \ell(Q)\right)\right) \\
& \leq c_{1}^{\gamma_{l}+1} \phi\left(Q\left(c_{Q}+l \ell(Q), \ell(Q) 2^{\gamma_{l}+1}\right)\right)
\end{aligned}
$$

and

$$
\phi(Q)=\phi\left(Q\left(c_{Q}, \ell(Q)\right)\right) \geq\left(\frac{1}{\widetilde{c}_{1}}\right)^{\gamma_{l}+1} \phi\left(Q\left(c_{Q}, \ell(Q) 2^{\gamma_{l}+1}\right)\right),
$$

which, combined with the condition $(\mathbf{S 2})$ of $\phi$, implies that

$$
\begin{aligned}
\frac{\phi(Q+l \ell(Q))}{\phi(Q)} & \leq \frac{c_{1}^{\gamma_{l}+1} \phi\left(Q\left(c_{Q}+l \ell(Q), \ell(Q) 2^{\gamma_{l}+1}\right)\right)}{\left(\widetilde{c}_{1}\right)^{-\gamma_{l}-1} \phi\left(Q\left(c_{Q}, \ell(Q) 2^{\gamma_{l}+1}\right)\right)} \\
& \leq c_{2} c_{1}^{\gamma_{l}+1}\left(\widetilde{c}_{1}\right)^{\gamma_{l}+1} \sim|l|^{\log _{2}\left(c_{1} \widetilde{c}_{1}\right)} .
\end{aligned}
$$

This, together with (2.2), then finishes the proof of (ii) and hence Lemma 2.6.

Lemma 2.7. Let $p(\cdot) \in C^{\log }\left(\mathbb{R}^{n}\right)$. Then there exists a positive constant $C$ such that, for all dyadic cubes $Q_{j k}$ with $j \in \mathbb{Z}_{+}$and $k \in \mathbb{Z}^{n}$,

$$
\begin{aligned}
\frac{1}{C} 2^{-\frac{n}{p_{-}} j}(1+|k|)^{n\left(\frac{1}{p_{+}}-\frac{1}{p_{-}}\right)} & \leq\left\|\chi_{Q_{j k}}\right\|_{L^{p(\cdot)}\left(\mathbb{R}^{n}\right)} \\
& \leq C 2^{-\frac{n}{p_{+}} j}(1+|k|)^{n\left(\frac{1}{p_{-}}-\frac{1}{p_{+}}\right)} .
\end{aligned}
$$

Proof. Let $Q_{00}$ be the dyadic cube $Q_{j k}$ with $j=0$ and $k=(0, \ldots, 0) \in \mathbb{Z}^{n}$. For any $j \in \mathbb{Z}_{+}$and $k \in \mathbb{Z}^{n}$, it is easy to see that $Q_{j k} \subset 2(1+|k|) Q_{00}$. Then, from [82, Lemma 2.6], we deduce that

$$
\left\|\chi_{Q_{j k}}\right\|_{L^{p(\cdot)}\left(\mathbb{R}^{n}\right)} \lesssim\left[\frac{\left|Q_{j k}\right|}{\left|2(1+|k|) Q_{00}\right|}\right]^{\frac{1}{p_{+}}}\left\|\chi_{2(1+|k|) Q_{00}}\right\|_{L^{p(\cdot)}\left(\mathbb{R}^{n}\right)}
$$




$$
\begin{aligned}
& \lesssim\left[\frac{\left|Q_{j k}\right|}{\left|2(1+|k|) Q_{00}\right|}\right]^{\frac{1}{p_{+}}}\left[\frac{\left|2(1+|k|) Q_{00}\right|}{\left|Q_{00}\right|}\right]^{\frac{1}{p_{-}}}\left\|\chi_{Q_{00}}\right\|_{L^{p(\cdot)}\left(\mathbb{R}^{n}\right)} \\
& \sim 2^{-\frac{n}{p_{+}} j}(1+|k|)^{n\left(\frac{1}{p_{-}}-\frac{1}{p_{+}}\right)}
\end{aligned}
$$

and, similarly,

$$
\left\|\chi_{Q_{j k}}\right\|_{L^{p(\cdot)}\left(\mathbb{R}^{n}\right)} \gtrsim 2^{-\frac{n}{p_{-}} j}(1+|k|)^{n\left(\frac{1}{p_{+}}-\frac{1}{p_{-}}\right)},
$$

which completes the proof of (2.3) and hence Lemma 2.7.

In what follows, for $h \in \mathcal{S}\left(\mathbb{R}^{n}\right)$ and $M \in \mathbb{Z}_{+}$, let

$$
\|h\|_{\mathcal{S}_{M}\left(\mathbb{R}^{n}\right)}:=\sup _{|\gamma| \leq M} \sup _{x \in \mathbb{R}^{n}}\left|\partial^{\gamma} h(x)\right|(1+|x|)^{n+M+\gamma} .
$$

Proof of Lemma 2.5. To prove this lemma, it suffices to show that there exists an $M \in \mathbb{N}$ such that, for all $t \in f_{p(\cdot), q(\cdot)}^{s(\cdot)}\left(\mathbb{R}^{n}\right)$ and $h \in \mathcal{S}\left(\mathbb{R}^{n}\right)$,

$$
\left|\left\langle T_{\psi} t, h\right\rangle\right| \lesssim\|t\|_{f_{p(\cdot), q(\cdot),(\cdot)}^{s(\cdot)}\left(\mathbb{R}^{n}\right)}\|h\|_{\mathcal{S}_{M}\left(\mathbb{R}^{n}\right)} .
$$

Indeed, by Remark 1.1(iv), we see that, for any $Q \in \mathcal{Q}^{*}$,

$$
\begin{aligned}
& \left|t_{Q}\right|=\left\|t_{Q} \chi_{Q}\right\|_{L^{p(\cdot)}(Q)}\left\|\chi_{Q}\right\|_{L^{p(\cdot)}(Q)}^{-1} \\
& \leq\left\|\left\{\sum_{\substack{\tilde{Q} \subset Q \\
\tilde{Q} \in \mathcal{Q}^{*}}}\left[|\widetilde{Q}|^{-\frac{s(\cdot)}{n}-\frac{1}{2}}\left|t_{\widetilde{Q}}\right| \chi_{\widetilde{Q}}\right]^{q(\cdot)}\right\}^{\frac{1}{q(\cdot)}}\right\|_{L^{p(\cdot)}(Q)}\left\|\chi_{Q}\right\|_{L^{p(\cdot)}(Q)}^{-1}|Q|^{\frac{s_{-}}{n}+\frac{1}{2}} \\
& \leq\|t\|_{f_{p(\cdot), q(\cdot)}^{s(\cdot),\left(\mathbb{R}^{n}\right)}}\left\|\chi_{Q}\right\|_{L^{p(\cdot)}(Q)}^{-1} \phi(Q)|Q|^{\frac{s_{-}}{n}+\frac{1}{2}},
\end{aligned}
$$

which implies that

$$
\begin{aligned}
\left|\left\langle T_{\psi} t, h\right\rangle\right| \leq \sum_{\ell(Q)=1}\left|t_{Q}\left\|\left\langle\Psi_{Q}, h\right\rangle\left|+\sum_{\ell(Q)<1}\right| t_{Q}\right\|\left\langle\psi_{Q}, h\right\rangle\right| \\
\leq\|t\|_{f_{p(\cdot), q(\cdot)}^{s(\cdot),}}\left\{\mathbb{R}^{n}\right) \\
\quad+\sum_{\ell(Q)=1}\left\|\chi_{Q}\right\|_{L^{p(\cdot)}(Q)}^{-1} \phi(Q)\left|\left\langle\Psi_{Q}, h\right\rangle\right| \\
\left.\quad|Q|^{\frac{s_{-}}{n}+\frac{1}{2}}\left\|\chi_{Q}\right\|_{L^{p(\cdot)}(Q)}^{-1} \phi(Q)\left|\left\langle\psi_{Q}, h\right\rangle\right|\right\}=: \mathrm{I}_{1}+\mathrm{I}_{2} .
\end{aligned}
$$

Let $M \in \mathbb{N}$ be such that

$$
M>2 \max \left\{\log _{2}\left(c_{1} \widetilde{c}_{1}\right)+n\left(\frac{1}{p_{-}}-\frac{1}{p_{+}}\right)+\frac{n}{2}, \log _{2} c_{1}+\frac{n}{p_{-}}-s_{-}-2 n\right\} .
$$

Then, for $\mathrm{I}_{1}$, by Lemmas 2.6 and 2.7 , we find that

$$
\mathrm{I}_{1} \leq\|f\|_{f_{p(\cdot), q(\cdot)}^{s(\cdot),\left(\mathbb{R}^{n}\right)}} \sum_{k \in \mathbb{Z}^{n}}\left\|\chi_{Q_{0 k}}\right\|_{L^{p(\cdot)}\left(Q_{0 k}\right)}^{-1} \phi\left(Q_{0 k}\right)\left|\left\langle\Psi_{Q_{0 k}}, h\right\rangle\right|
$$




$$
\begin{aligned}
& \lesssim\|f\|_{f_{p(\cdot), q(\cdot)}^{s(\cdot),\left(\mathbb{R}^{n}\right)}}\|h\|_{\mathcal{S}_{M}\left(\mathbb{R}^{n}\right)} \sum_{k \in \mathbb{Z}^{n}}(1+|k|)^{n\left(\frac{1}{p_{-}}-\frac{1}{p_{+}}\right)+\log _{2}\left(c_{1} \widetilde{c}_{1}\right)-n-M} \\
& \lesssim\|f\|_{f_{p(\cdot), q(\cdot)}^{s(\cdot),}\left(\mathbb{R}^{n}\right)}\|h\|_{\mathcal{S}_{M}\left(\mathbb{R}^{n}\right)} .
\end{aligned}
$$

On the other hand, for $\mathrm{I}_{2}$, by [80, Lemma 2.4] and Lemmas 2.6 and 2.7, we conclude that

$$
\begin{aligned}
\mathrm{I}_{2} \leq\|f\|_{f_{p(\cdot), q(\cdot)}^{s(\cdot),\left(\mathbb{R}^{n}\right)}}\|h\|_{\mathcal{S}_{M}\left(\mathbb{R}^{n}\right)} \sum_{j=1}^{\infty} \sum_{k \in \mathbb{Z}^{n}} 2^{-j\left(s_{-}+\frac{n}{2}-\log _{2} c_{1}-\frac{n}{p_{-}}\right)} 2^{-j M} \\
\quad \times(1+|k|)^{n\left(\frac{1}{p_{-}}-\frac{1}{p_{+}}\right)+\log _{2}\left(c_{1} \widetilde{c}_{1}\right)} \frac{1}{\left(1+\left|2^{-j} k\right|\right)^{n+M}} \\
\lesssim\|f\|_{f_{p(\cdot), q(\cdot)}^{s(\cdot),}\left(\mathbb{R}^{n}\right)}\|h\|_{\mathcal{S}_{M}\left(\mathbb{R}^{n}\right)},
\end{aligned}
$$

which, together with the estimate of $\mathrm{I}_{1}$, implies that

$$
\left|\left\langle T_{\psi} t, h\right\rangle\right| \lesssim\|t\|_{f_{p(\cdot), q(\cdot)}^{s(\cdot),}\left(\mathbb{R}^{n}\right)}\|h\|_{\mathcal{S}_{M}\left(\mathbb{R}^{n}\right)} .
$$

Thus,

$$
T_{\psi} t=\sum_{Q \in \mathcal{Q}^{*}, \ell(Q)=1} t_{Q} \Psi_{Q}+\sum_{Q \in \mathcal{Q}^{*}, \ell(Q)<1} t_{Q} \psi_{Q}
$$

converges in $\mathcal{S}^{\prime}\left(\mathbb{R}^{n}\right)$ and $T_{\psi}: f_{p(\cdot), q(\cdot)}^{s(\cdot),}\left(\mathbb{R}^{n}\right) \rightarrow \mathcal{S}^{\prime}\left(\mathbb{R}^{n}\right)$ is continuous, which completes the proof of Lemma 2.5.

For a sequence $t=\left\{t_{Q}\right\}_{Q \in \mathcal{Q}^{*}} \subset \mathbb{C}, r \in(0, \infty)$ and $\lambda \in(0, \infty)$, let

$$
\left(t_{r, \lambda}^{*}\right)_{Q}:=\left\{\sum_{R \in \mathcal{Q}^{*}, \ell(R)=\ell(Q)} \frac{\left|t_{R}\right|^{r}}{\left[1+\ell(R)^{-1}\left|x_{R}-x_{Q}\right|^{\lambda}\right.}\right\}^{\frac{1}{r}}, \quad Q \in \mathcal{Q}^{*},
$$

and $t_{r, \lambda}^{*}:=\left\{\left(t_{r, \lambda}^{*}\right)_{Q}\right\}_{Q \in \mathcal{Q}^{*}}$.

We have the following estimates.

Lemma 2.8. Let $p, q, s$ and $\phi$ be as in Definition $1.4, r \in\left(0, \min \left\{p_{-}, q_{-}\right\}\right)$and

$$
\lambda \in\left(n+C_{\log }(s)+r \log _{2}\left(c_{1} \widetilde{c}_{1}\right), \infty\right),
$$

where $c_{1}$ and $\widetilde{c}_{1}$ are as in the condition (S1) and $C_{\log }(s)$ is as in (1.1) with $g$ replaced by $s$. Then there exists a constant $C \in[1, \infty)$ such that, for all $t \in f_{p(\cdot), q(\cdot)}^{s(\cdot), \phi}\left(\mathbb{R}^{n}\right)$

$$
\|t\|_{f_{p(\cdot), q(\cdot)}^{s(\cdot),}\left(\mathbb{R}^{n}\right)} \leq\left\|t_{r, \lambda}^{*}\right\|_{f_{p(\cdot), q(\cdot)}^{s(\cdot), \phi}\left(\mathbb{R}^{n}\right)} \leq C\|t\|_{f_{p(\cdot), q(\cdot)}^{s(\cdot),}\left(\mathbb{R}^{n}\right)^{\circ}}
$$

To prove Lemma 2.8, we need Lemma 2.9 below, which is just [20, Theorem 3.2] (the vector-valued convolution inequality) and plays a key role throughout this article. In what follows, for any $m \in(0, \infty)$ and $j \in \mathbb{Z}$, let

$$
\eta_{j, m}(x):=2^{j n}\left(1+2^{j}|x|\right)^{-m}, \quad x \in \mathbb{R}^{n} .
$$


Lemma 2.9. Let $p, q \in C^{\log }\left(\mathbb{R}^{n}\right)$ satisfy $1<p_{-} \leq p_{+}<\infty$ and $1<q_{-} \leq q_{+}<$ $\infty$. Let $m \in(n, \infty)$. Then there exists a positive constant $C$ such that, for all sequences $\left\{f_{j}\right\}_{j \in \mathbb{N}} \subset L_{\text {loc }}^{1}\left(\mathbb{R}^{n}\right)$,

$$
\left\|\left\{\sum_{j \in \mathbb{N}}\left|\eta_{j, m} * f_{j}\right|^{q(\cdot)}\right\}^{\frac{1}{q(\cdot)}}\right\|_{L^{p(\cdot)}\left(\mathbb{R}^{n}\right)} \leq C\left\|\left\{\sum_{j \in \mathbb{N}}\left|f_{j}\right|^{q(\cdot)}\right\}^{\frac{1}{q(\cdot)}}\right\|_{L^{p(\cdot)}\left(\mathbb{R}^{n}\right)} .
$$

The following Lemma 2.10 is just [36, Lemma 19] (see also [20, Lemma 6.1]).

Lemma 2.10. Let $s \in C_{\operatorname{loc}}^{\log }\left(\mathbb{R}^{n}\right)$ and $L \in\left[C_{\log }(s), \infty\right)$, where $C_{\log }(s)$ is as in (1.1) with $g$ replaced by s. Then there exists a positive constant $C$ such that, for all $x, y \in \mathbb{R}^{n}, m \in(0, \infty)$ and $v \in \mathbb{Z}_{+}$,

$$
2^{v s(x)} \eta_{v, m+L}(x-y) \leq C 2^{v s(y)} \eta_{v, m}(x-y) .
$$

Proof of Lemma 2.8. Notice that, for all $Q \in \mathcal{Q}^{*},\left|t_{Q}\right| \leq\left(t_{r, \lambda}^{*}\right)_{Q}$. This immediately implies that $\|t\|_{f_{p(\cdot), q(\cdot)}^{s(\cdot),}\left(\mathbb{R}^{n}\right)} \leq\left\|t_{r, \lambda}^{*}\right\|_{f_{p(\cdot), q(\cdot)}^{s(\cdot),}\left(\mathbb{R}^{n}\right)}$, since $f_{p(\cdot), q(\cdot)}^{s(\cdot),}\left(\mathbb{R}^{n}\right)$ is a quasiBanach lattice (see Remark 2.2(i)).

Conversely, let $P$ be a given dyadic cube. For any $Q \in \mathcal{Q}^{*}$, let $v_{Q}:=t_{Q}$ if $Q \subset 3 P$ and $v_{Q}:=0$ otherwise, and let $u_{Q}:=t_{Q}-v_{Q}$. Set $v:=\left\{v_{Q}\right\}_{Q \in \mathcal{Q}^{*}}$ and $u:=\left\{u_{Q}\right\}_{Q \in \mathcal{Q}^{*}}$. Then, for all $Q \in \mathcal{Q}^{*}$, we have

$$
\left(t_{r, \lambda}^{*}\right)_{Q} \lesssim\left(v_{r, \lambda}^{*}\right)_{Q}+\left(u_{r, \lambda}^{*}\right)_{Q}
$$

From the proof of [20, Theorem 3.11], we deduce that, for all $t \in f_{p(\cdot), q(\cdot)}^{s(\cdot), 1}\left(\mathbb{R}^{n}\right)$,

$$
\left\|t_{r, \lambda}^{*}\right\|_{f_{p(\cdot), q(\cdot)}^{s(\cdot),}\left(\mathbb{R}^{n}\right)} \lesssim\|t\|_{f_{p(\cdot), q(\cdot)}^{s(\cdot), 1}\left(\mathbb{R}^{n}\right)},
$$

which implies that

$$
\begin{aligned}
\mathrm{I}_{P} & :=\frac{1}{\phi(P)}\left\|\left[\sum_{Q \subset P, Q \in \mathcal{Q}^{*}}\left\{|Q|^{-\left[\frac{s(\cdot)}{n}+\frac{1}{2}\right]}\left(v_{r, \lambda}^{*}\right)_{Q} \chi_{Q}\right\}^{q(\cdot)}\right]^{\frac{1}{q(\cdot)}}\right\|_{L^{p(\cdot)}(P)} \\
& \left.\lesssim \frac{1}{\phi(P)}\left\|v_{r, \lambda}^{*}\right\|_{f_{p(\cdot), q(\cdot)}^{s(\cdot), 1}\left(\mathbb{R}^{n}\right)} \lesssim \frac{1}{\phi(P)}\|v\|_{f_{p(\cdot), q(\cdot)}^{s(\cdot),}\left(\mathbb{R}^{n}\right)}\left\{|Q|^{-\left[\frac{s(\cdot)}{n}+\frac{1}{2}\right]}\left|t_{Q}\right| \chi_{Q}\right\}^{q(\cdot)}\right]^{\frac{1}{q(\cdot)}}\|\|_{L^{p(\cdot)}(3 P)} .
\end{aligned}
$$

By this and the condition $(\mathbf{S 1})$ of $\phi$, we conclude that

$$
\left\|v_{r, \lambda}^{*}\right\|_{f_{p(\cdot), q(\cdot)}^{s(\cdot),}\left(\mathbb{R}^{n}\right)} \leq \sup _{P \in \mathcal{Q}} \mathrm{I}_{P} \lesssim\|t\|_{f_{p(\cdot), q(\cdot)}^{s(\cdot),}\left(\mathbb{R}^{n}\right)} .
$$

Next, we deal with $u$. To this end, let, for $i \in \mathbb{Z}_{+}$and $k \in \mathbb{Z}^{n}$,

$$
A(i, k, P):=\left\{\widetilde{Q} \in \mathcal{Q}^{*}: \ell(\widetilde{Q})=2^{-i} \ell(P), \widetilde{Q} \subset P+k \ell(P)\right\} .
$$


Then, we have

$$
\begin{aligned}
& \mathrm{J}_{P}:=\frac{1}{\phi(P)}\left\|\left[\sum_{Q \subset P, Q \in \mathcal{Q}^{*}}\left\{|Q|^{-\left[\frac{s(\cdot)}{n}+\frac{1}{2}\right]}\left(u_{r, \lambda}^{*}\right)_{Q} \chi_{Q}\right\}^{q(\cdot)}\right]^{\frac{1}{q(\cdot)}}\right\|_{L^{p(\cdot)}(P)} \\
& =\frac{1}{\phi(P)} \|\left\{\sum _ { i = 0 } ^ { \infty } \sum _ { \substack { Q \subset P , Q \in \mathcal { Q } ^ { * } \\
\ell ( Q ) = 2 ^ { - i } \ell ( P ) } } \left(|Q|^{-\left[\frac{s(\cdot)}{n}+\frac{1}{2}\right]}\right.\right. \\
& \left.\left.\times\left\{\sum_{\substack{k \in \mathbb{Z}^{n} \\
|k| \geq 2}} \sum_{R \in A(i, k, P)} \frac{\left|u_{R}\right|^{r}}{\left[1+\ell(Q)^{-1}\left|x_{R}-x_{Q}\right|^{\lambda}\right.}\right\}^{\frac{1}{r}} \chi_{Q}\right)^{q(\cdot)}\right\}^{\frac{1}{q(\cdot)}} \|_{L^{p(\cdot)}(P)} .
\end{aligned}
$$

Notice that, when $x \in Q, y \in R$ and $\ell(R)=\ell(Q)$,

$$
1+[\ell(Q)]^{-1}|x-y| \sim 1+[\ell(Q)]^{-1}\left|x_{Q}-x_{R}\right| .
$$

From this, we deduce that, for all $x \in Q, \mu \in(0, \infty), j \in \mathbb{Z}_{+}$and $k \in \mathbb{Z}^{n}$,

$$
\begin{aligned}
\eta_{j_{Q}, \mu} *\left(\left[\sum_{R \in A(i, k, P)}\left|u_{R}\right| \chi_{R}\right]^{r}\right)(x) & =\sum_{R \in A(i, k, P)} \int_{R} \frac{2^{n j_{Q}}\left|u_{R}\right|^{r}}{\left(1+2^{j}|x-y|\right)^{\mu}} d y \\
& \sim \sum_{R \in A(i, k, P)} \frac{\left|u_{R}\right|^{r}}{\left[1+\ell(Q)^{-1}\left|x_{Q}-x_{R}\right|\right]^{\mu}} .
\end{aligned}
$$

Since

$$
\lambda>n+C_{\log }(s)+r \log _{2}\left(c_{1} \widetilde{c}_{1}\right),
$$

it follows that there exist $m \in(n, \infty)$ and $L \in\left(C_{\log }(s), \infty\right)$ such that

$$
\lambda>m+L+r \log _{2}\left(c_{1} \widetilde{c}_{1}\right) .
$$

Observe that, when $|k| \geq 2, i \in \mathbb{Z}_{+}, Q \subset P$ with $\ell(Q)=2^{-i} \ell(P)$ and $R \in$ $A(i, k, P)$,

$$
1+[\ell(Q)]^{-1}\left|x_{Q}-x_{R}\right| \sim 2^{i}|k|
$$

Then, by Lemma 2.10, we conclude that

$$
\begin{aligned}
\mathrm{J}_{P} \lesssim \frac{1}{\phi(P)} & \|\left\{\sum _ { i = 0 } ^ { \infty } \left(\sum _ { \substack { \ell ( Q ) = 2 ^ { - i } \ell ( P ) \\
Q \in \mathcal { Q } ^ { * } , Q \subset P } } \left\{\sum_{k \in \mathbb{Z}^{n},|k| \geq 2}\left(2^{i}|k|\right)^{m+L-\lambda}|Q|^{-\left[\frac{s(\cdot)}{n}+\frac{1}{2}\right] r}\right.\right.\right. \\
& \left.\left.\left.\times \eta_{j_{Q}, m+L} *\left(\left[\sum_{R \in A(i, k, P)}\left|u_{R}\right| \chi_{R}\right]^{r}\right)\right\}^{\frac{1}{r}} \chi_{Q}\right)^{q(\cdot)}\right\}^{\frac{1}{q(\cdot)}} \|_{L^{p(\cdot)}(P)}
\end{aligned}
$$




$$
\begin{aligned}
& \lesssim \frac{1}{\phi(P)} \|\left\{\sum _ { i = 0 } ^ { \infty } \left[\sum_{k \in \mathbb{Z}^{n},|k| \geq 2}\left(2^{i}|k|\right)^{m+L-\lambda}\right.\right. \\
& \left.\left.\left.\quad \times \eta_{i+j_{P}, m} *\left(\left\{\sum_{R \in A(i, k, P)}|R|^{-\left[\frac{s(\cdot)}{n}+\frac{1}{2}\right]}\left|u_{R}\right| \chi_{R}\right\}\right)\right]^{r}\right\}\right\}_{L^{p(\cdot)}(P)}^{\frac{q(\cdot)}{r}},
\end{aligned}
$$

which, combined with Remark $1.1(\mathrm{i})$ and the fact that, for all $d \in[0,1]$ and $\left\{\theta_{j}\right\}_{j} \subset \mathbb{C}$,

$$
\left(\sum_{j}\left|\theta_{j}\right|\right)^{d} \leq \sum_{j}\left|\theta_{j}\right|^{d},
$$

implies that

$$
\begin{aligned}
\mathrm{J}_{P} \lesssim & \frac{1}{\phi(P)} \| \sum_{i=0}^{\infty} \sum_{k \in \mathbb{Z}^{n},|k| \geq 2}\left(2^{i}|k|\right)^{m+L-\lambda} \\
& \times \eta_{i+j_{P}, m} *\left(\left\{\sum_{R \in A(i, k, P)}|R|^{-\left[\frac{s(\cdot)}{n}+\frac{1}{2}\right]}\left|u_{R}\right| \chi_{R}\right\}\right) \|_{L^{\frac{p(\cdot)}{r}}(P)}^{r} \\
\lesssim & \frac{1}{\phi(P)}\left\{\sum_{i=0}^{\infty} \sum_{k \in \mathbb{Z}^{n},|k| \geq 2}\left(2^{i}|k|\right)^{m+L-\lambda} \|^{\frac{1}{r}}\right. \\
& \left.\times\left\|\eta_{i+j_{P}, m} *\left(\left\{\sum_{R \in A(i, k, P)}|R|^{-\left[\frac{s(\cdot)}{n}+\frac{1}{2}\right]}\left|u_{R}\right| \chi_{R}\right\}\right)\right\|_{L^{\frac{p(\cdot)}{r}}(P)}\right\}^{\frac{1}{r}} .
\end{aligned}
$$

From this, $m \in(n, \infty)$, Lemma 2.9, Lemma 2.6(ii) and

$$
\lambda>m+L+r \log _{2}\left(c_{1} \widetilde{c}_{1}\right),
$$

we deduce that

$$
\begin{aligned}
\mathrm{J}_{P} & \lesssim \frac{1}{\phi(P)}\left\{\sum_{i=0}^{\infty} \sum_{\substack{k \in \mathbb{Z}^{n} \\
|k| \geq 2}}\left(2^{i}|k|\right)^{m+L-\lambda}\left\|\sum_{R \in A(i, k, P)}|R|^{-\left[\frac{s(\cdot)}{n}+\frac{1}{2}\right]}\left|u_{R}\right| \chi_{R}\right\|_{L^{p(\cdot)}\left(\mathbb{R}^{n}\right)}^{r}\right\} \\
& \lesssim\left\{\sum_{i=0}^{\infty} \sum_{\substack{k \in \mathbb{Z}^{n} \\
|k| \geq 2}}\left(2^{i}|k|\right)^{m+L-\lambda}\left[\frac{\phi(P+k \ell(P))}{\phi(P)}\right]^{\frac{1}{r}}\|t\|_{f_{p(\cdot), q(\cdot)}^{s(\cdot),}\left(\mathbb{R}^{n}\right)}^{\frac{1}{r}}\right. \\
& \lesssim\left\{\sum_{i=0}^{\infty} \sum_{\substack{k \in \mathbb{Z}^{n} \\
|k| \geq 2}} 2^{i(m+L-\lambda)}|k|^{m+L-\lambda}|k|^{r \log \left(c_{1} \widetilde{c}_{1}\right)}\right\}^{\frac{1}{r}}\|t\|_{f_{p(\cdot), q(\cdot)}^{s(\cdot)}\left(\mathbb{R}^{n}\right)} \sim\|t\|_{f_{p(\cdot), q(\cdot)}^{s(\cdot),\left(\mathbb{R}^{n}\right)}} .
\end{aligned}
$$


This, together with the arbitrariness of $P \in \mathcal{Q}$, further implies that

$$
\left\|u_{r, \lambda}^{*}\right\|_{f_{p(\cdot), q(\cdot)}^{s(\cdot),\left(\mathbb{R}^{n}\right)}} \lesssim\|t\|_{f_{p(\cdot), q(\cdot)}^{s(\cdot),}\left(\mathbb{R}^{n}\right)} .
$$

Combining (2.4), (2.5) and (2.7), we conclude that

$$
\left\|t_{r, \lambda}^{*}\right\|_{f_{p(\cdot), q(\cdot)}^{s(\cdot),}\left(\mathbb{R}^{n}\right)} \lesssim\|t\|_{f_{p(\cdot), q(\cdot)}^{s(\cdot),}\left(\mathbb{R}^{n}\right)}
$$

which completes the proof of Lemma 2.8.

Now we give the proof of Theorem 2.3.

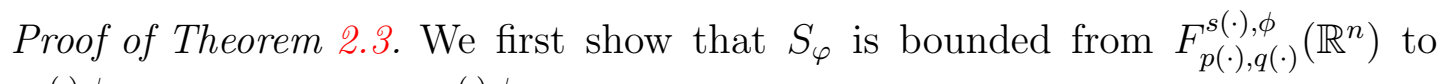
$f_{p(\cdot), q(\cdot)}^{s(\cdot),}\left(\mathbb{R}^{n}\right)$. Let $f \in F_{p(\cdot), q(\cdot)}^{s(\cdot),}\left(\mathbb{R}^{n}\right)$. From [20, Lemma A.6] (the $r$-trick lemma) and its proof, we deduce that, for all $L \in\left[C_{\log }(s), \infty\right), m \in\left(n+\log _{2} c_{1}, \infty\right)$,

$$
r \in\left(0, \min \left\{1, p_{-}, q_{-}\right\}\right)
$$

and $x \in Q:=Q_{j k} \in \mathcal{Q}^{*}$,

$$
\sup _{z \in Q}\left|\varphi_{j} * f(z)\right|^{r} \lesssim 2^{j n} \sum_{l \in \mathbb{Z}^{n}} \int_{Q_{j(k+l)}}\left(1+2^{j}|x-y|\right)^{-(2 m+L)}\left|\varphi_{j} * f(y)\right|^{r} d y,
$$

which implies that

$$
\begin{aligned}
& \left\|S_{\varphi} f\right\|_{f_{p(\cdot), q(\cdot)}^{s(\cdot),}\left(\mathbb{R}^{n}\right)} \\
& \lesssim \sup _{P \in \mathcal{Q}} \frac{1}{\phi(P)} \|\left[\sum _ { j = ( j _ { P } \vee 0 ) } ^ { \infty } \left\{\sum_{k \in \mathbb{Z}^{n}} 2^{j s(\cdot)}\right.\right. \\
& \left.\left.\quad \times\left[2^{j n} \sum_{l \in \mathbb{Z}^{n}} \int_{Q_{j(k+l)}} \frac{\left|\varphi_{j} * f(y)\right|^{r}}{\left(1+2^{j}|\cdot-y|\right)^{2 m+L}} d y\right]^{\frac{1}{r}} \chi_{Q_{j k}}\right\}^{q(\cdot)}\right]^{\frac{1}{q(\cdot)}} \|_{L^{p(\cdot)}(P)} \\
& \lesssim \sup _{P \in \mathcal{Q}} \frac{1}{\phi(P)} \|\left[\sum _ { j = ( j _ { P } \vee 0 ) } ^ { \infty } \left\{\sum_{k \in \mathbb{Z}^{n}} 2^{j s(\cdot)} \sum_{l \in \mathbb{Z}^{n}}(1+|l|)^{-m}\right.\right. \\
& \left.\left.\quad \times\left[\eta_{j, m+L} *\left(\left|\varphi_{j} * f \chi_{Q_{j(k+l)}}\right|^{r}\right)\right] \chi_{Q_{j k}}\right\}^{\frac{q(\cdot)}{r}}\right]^{\frac{1}{q(\cdot)}} \|_{L^{p(\cdot)}(P)}
\end{aligned}
$$

where the last inequality comes from the fact that, when $x \in Q_{j k}$ and $y \in Q_{j(k+l)}$,

$$
1+2^{j}|x-y| \sim 1+|l| .
$$

Observe that, for any given $P \in \mathcal{Q}$, if $Q_{j k} \subset P$, then $Q_{j(k+l)} \subset 3 n|l| P$ for all $l \in \mathbb{Z}^{n}$. By this and (2.8), we see that

$\left\|S_{\varphi} f\right\|_{f_{p(\cdot), q(\cdot)}^{s(\cdot),}\left(\mathbb{R}^{n}\right)} \lesssim \sup _{P \in \mathcal{Q}} \frac{1}{\phi(P)} \|\left[\sum_{j=\left(j_{P} \vee 0\right)}^{\infty}\left\{2^{j r s(\cdot)}\right.\right.$ 


$$
\left.\left.\times\left[\sum_{l \in \mathbb{Z}^{n}}(1+|l|)^{-m} \eta_{j, m+L} *\left(\left|\varphi_{j} * f \chi_{3 n|l| P}\right|^{r}\right)\right]\right\}^{\frac{q(\cdot)}{r}}\right]^{\frac{1}{q(\cdot)}} \|_{L^{p(\cdot)}(P)}
$$

which, combined with the Minkowski inequality, Lemma 2.10 and Remark 1.1(i), further implies that

$$
\begin{aligned}
& \left\|S_{\varphi} f\right\|_{f_{p(\cdot), q(\cdot)}^{s(\cdot),}\left(\mathbb{R}^{n}\right)} \\
& \lesssim \sup _{P \in \mathcal{Q}} \frac{1}{\phi(P)}\left\{\sum_{l \in \mathbb{Z}^{n}}(1+|l|)^{-m}\right. \\
& \left.\quad \times\left\|\left(\sum_{j=\left(j_{P} \vee 0\right)}^{\infty}\left[\eta_{j, m+L} *\left(\left|2^{j s(\cdot)} \varphi_{j} * f \chi_{3 n|l| P}\right|^{r}\right)\right]^{\frac{q(\cdot)}{r}}\right)^{\frac{r}{q(\cdot)} \|}\right\|_{L^{\frac{p(\cdot)}{r}(P)}}\right\}^{\frac{1}{r}} .
\end{aligned}
$$

From this, Lemma 2.9, $m \in\left(n+\log _{2} c_{1}, \infty\right)$ and the condition $(\mathbf{S 1})$ of $\phi$, it follows that

$$
\begin{aligned}
& \left\|S_{\varphi} f\right\|_{f_{p(\cdot), q(\cdot)}^{s(\cdot),}\left(\mathbb{R}^{n}\right)} \\
& \lesssim \sup _{P \in \mathcal{Q}} \frac{1}{\phi(P)}\left\{\sum_{l \in \mathbb{Z}^{n}}(1+|l|)^{-m}\left\|\left[\sum_{j=\left(j_{P} \vee 0\right)}^{\infty}\left(2^{j s(\cdot)}\left|\varphi_{j} * f\right|\right)^{q(\cdot)}\right]^{\frac{1}{q(\cdot)}}\right\|_{L^{p(\cdot)(3 n|l| P)}}^{r}\right\}^{\frac{1}{r}} \\
& \lesssim\|f\|_{F_{p(\cdot), q(\cdot)}^{s(\cdot),}\left(\mathbb{R}^{n}\right)}\left[\sum_{l \in \mathbb{Z}^{n}}(1+|l|)^{-m}(1+|l|)^{\log _{2} c_{1}}\right]^{\frac{1}{r}} \lesssim\|f\|_{F_{p(\cdot), q(\cdot)}^{s(\cdot),}\left(\mathbb{R}^{n}\right)},
\end{aligned}
$$

which implies that $S_{\varphi}$ is bounded from $F_{p(\cdot), q(\cdot)}^{s(\cdot),}\left(\mathbb{R}^{n}\right)$ to $f_{p(\cdot), q(\cdot)}^{s(\cdot),}\left(\mathbb{R}^{n}\right)$.

By repeating the argument used in the proof of [80, Theorem 2.1], with [80, Lemmas 2.7 and 2.8] therein replaced by Lemmas 2.5 and 2.8 here, we conclude that $T_{\psi}$ is bounded from $f_{p(\cdot), q(\cdot)}^{s(\cdot),}\left(\mathbb{R}^{n}\right)$ to $F_{p(\cdot), q(\cdot)}^{s(\cdot),}\left(\mathbb{R}^{n}\right)$, the details being omitted. Finally, by the Calderón reproducing formula (see, for example, [80, Lemma 2.3]), we know that $T_{\psi} \circ S_{\varphi}$ is the identity on $F_{p(\cdot), q(\cdot)}^{s(\cdot),}\left(\mathbb{R}^{n}\right)$, which completes the proof of Theorem 2.3.

\section{Several Equivalent CharaCterizations of $F_{p(\cdot), q(\cdot)}^{s(\cdot),}\left(\mathbb{R}^{n}\right)$}

In this section, we first establish molecular and atomic characterizations for $F_{p(\cdot), q(\cdot)}^{s(\cdot),}\left(\mathbb{R}^{n}\right)$ via Sobolev embeddings. Secondly, we characterize $F_{p(\cdot), q(\cdot)}^{s(\cdot), \phi}\left(\mathbb{R}^{n}\right)$ in terms of the Peetre maximal function, which is further applied to show that

$$
\mathcal{S}\left(\mathbb{R}^{n}\right) \hookrightarrow F_{p(\cdot), q(\cdot)}^{s(\cdot),}\left(\mathbb{R}^{n}\right) \hookrightarrow \mathcal{S}^{\prime}\left(\mathbb{R}^{n}\right)
$$

and give out two equivalent quasi-norms of $F_{p(\cdot), q(\cdot)}^{s(\cdot), \phi}\left(\mathbb{R}^{n}\right)$, which may be useful in applications.

For notational simplicity, in what follows, for all $Q \in \mathcal{Q}^{*}$, let $\widetilde{\chi}_{Q}:=|Q|^{-1 / 2} \chi_{Q}$. 
Proposition 3.1. Let $\phi$ be a set function as in Definition 1.4, $s_{0}, s_{1}, p_{0}, p_{1}$ be measurable functions satisfying that, for all $x \in \mathbb{R}^{n},-\infty<s_{1}(x) \leq s_{0}(x)<\infty$, $0<p_{0}(x) \leq p_{1}(x)<\infty$ and

$$
s_{0}(x)-\frac{n}{p_{0}(x)}=s_{1}(x)-\frac{n}{p_{1}(x)} .
$$

Assume that $0<\left(p_{0}\right)_{-} \leq\left(p_{1}\right)_{-} \leq\left(p_{1}\right)_{+}<\infty$ and $s_{0}, \frac{1}{p_{0}} \in C_{\operatorname{loc}}^{\log }\left(\mathbb{R}^{n}\right)$. If $q(x)=\infty$ for all $x \in \mathbb{R}^{n}$ or $0<q_{-} \leq q(x)<\infty$ for all $x \in \mathbb{R}^{n}$, then

$$
f_{p_{0}(\cdot), q(\cdot)}^{s_{0}(\cdot),}\left(\mathbb{R}^{n}\right) \hookrightarrow f_{p_{1}(\cdot), q(\cdot)}^{s_{1}(\cdot),}\left(\mathbb{R}^{n}\right) .
$$

Proof. Let $t:=\left\{t_{Q}\right\}_{Q \in \mathcal{Q}^{*}} \in f_{p_{0}(\cdot), q(\cdot)}^{s_{0}(\cdot), \phi}\left(\mathbb{R}^{n}\right)$. We need to prove

$$
\|t\|_{f_{p_{1}(\cdot), q(\cdot)}^{s_{1}(\cdot), \phi}\left(\mathbb{R}^{n}\right)} \lesssim\|t\|_{f_{p_{0}(\cdot), q(\cdot)}^{s_{0}(\cdot),}}\left(\mathbb{R}^{n}\right) \cdot
$$

To this end, let $P \in \mathcal{Q}$ be any given dyadic cube. For all $Q \in \mathcal{Q}^{*}$, let $u_{Q}:=t_{Q}$ when $Q \subset P$ and $u_{Q}:=0$ otherwise. Then, by the Sobolev embedding theorem $\left(\left[72\right.\right.$, Theorem 3.1]), namely, $f_{p_{0}(\cdot), q(\cdot)}^{s_{0}(\cdot), 1}\left(\mathbb{R}^{n}\right) \hookrightarrow f_{p_{1}(\cdot), q(\cdot)}^{s_{1}(\cdot), 1}\left(\mathbb{R}^{n}\right)$, we conclude that

$$
\begin{aligned}
& \left\|\left\{\sum_{j=\left(j_{P} \vee 0\right)}^{\infty} \sum_{k \in \mathbb{Z}^{n}}\left[2^{j s_{1}(\cdot)}\left|t_{Q_{j k}}\right| \widetilde{\chi}_{Q_{j k}}\right]^{q(\cdot)}\right\}^{\frac{1}{q(\cdot)}}\right\|_{L^{p_{1}(\cdot)}(P)} \\
& =\left\|\left\{\sum_{j=0}^{\infty} \sum_{k \in \mathbb{Z}^{n}}\left[2^{j s_{1}(\cdot)}\left|u_{Q_{j k}}\right| \widetilde{\chi}_{Q_{j k}}\right]^{q(\cdot)}\right\}^{\frac{1}{q(\cdot)}}\right\|_{L^{p_{1}(\cdot)}\left(\mathbb{R}^{n}\right)}=\|u\|_{f_{p_{1}(\cdot), q(\cdot)}^{s_{1}(\cdot),}}\left(\mathbb{R}^{n}\right) \\
& \lesssim\|u\|_{f_{p_{0}(\cdot), q(\cdot)}^{s_{0}(\cdot),}\left(\mathbb{R}^{n}\right)} \sim\left\|\left\{\sum_{j=0}^{\infty} \sum_{k \in \mathbb{Z}^{n}}\left[2^{j s_{0}(\cdot)}\left|u_{Q_{j k}}\right| \widetilde{\chi}_{Q_{j k}}\right]^{q(\cdot)}\right\}^{\frac{1}{q(\cdot)}}\right\|_{L^{p_{0}(\cdot)}\left(\mathbb{R}^{n}\right)} \\
& \sim\left\|\left\{\sum_{j=\left(j_{P} \vee 0\right)}^{\infty} \sum_{k \in \mathbb{Z}^{n}}\left[2^{j s_{0}(\cdot)}\left|t_{Q_{j k}}\right| \widetilde{\chi}_{Q_{j k}}\right]^{q(\cdot)}\right\}^{\frac{1}{q(\cdot)}}\right\|_{L_{p_{0}(\cdot)(P)}},
\end{aligned}
$$

which implies that

$$
\begin{aligned}
\|t\|_{f_{p_{1}(\cdot), q(\cdot)}^{s_{1}(\cdot), \phi}\left(\mathbb{R}^{n}\right)} & \lesssim \sup _{P \in \mathcal{Q}} \frac{1}{\phi(P)}\left\|\left\{\sum_{j=\left(j_{P} \vee 0\right)}^{\infty} \sum_{k \in \mathbb{Z}^{n}}\left[2^{j s_{0}(\cdot)}\left|t_{Q_{j k}}\right| \widetilde{\chi}_{Q_{j k}}\right]^{q(\cdot)}\right\}^{\frac{1}{q(\cdot)}}\right\|_{L^{p_{0}(\cdot)(P)}} \\
& \sim\|t\|_{f_{p_{0}(\cdot), q(\cdot)}^{s_{0}(\cdot),}\left(\mathbb{R}^{n}\right)^{\cdot}}
\end{aligned}
$$

This finishes the proof of Proposition 3.1

Proposition 3.2. Let $\phi$ be a set function as in Definition 1.4, $s_{0}, s_{1}, p_{0}, p_{1}$ be measurable functions satisfying that, for all $x \in \mathbb{R}^{n},-\infty<s_{1}(x) \leq s_{0}(x)<\infty$, $0<p_{0}(x) \leq p_{1}(x)<\infty$ and

$$
s_{0}(x)-\frac{n}{p_{0}(x)}=s_{1}(x)-\frac{n}{p_{1}(x)} .
$$


Assume that $0<\left(p_{0}\right)_{-} \leq\left(p_{1}\right)_{+}<\infty, s_{0}, \frac{1}{p_{0}} \in C_{\text {loc }}^{\log }\left(\mathbb{R}^{n}\right)$ and

$$
\inf _{x \in \mathbb{R}^{n}}\left[s_{0}(x)-s_{1}(x)\right]>0 .
$$

Then, for all $q \in(0, \infty]$,

$$
f_{p_{0}(\cdot), \infty}^{s_{0}(\cdot), \phi}\left(\mathbb{R}^{n}\right) \hookrightarrow f_{p_{1}(\cdot), q}^{s_{1}(\cdot), \phi}\left(\mathbb{R}^{n}\right) .
$$

The proof of Proposition 3.2 is similar to that of Proposition 3.1, with [72, Theorem 3.1] replaced by [72, Theorem 3.2], the details being omitted.

Remark 3.3. (i) When $\phi(Q)=1$ for all cube $Q$, the conclusions of Propositions 3.1 and 3.2 are just [72, Theorem 3.1] and [72, Theorem 3.2], respectively.

(ii) When $p, q, s$ and $\phi$ are as in Remark 1.5(ii), Proposition 3.2 goes back to [80, Proposition 2.5].

Combining Theorem 2.3 and Proposition 3.1, we immediately obtain the following Corollary 3.4, the details being omitted.

Corollary 3.4. Let $i \in\{0,1\}, p_{i}, q \in \mathcal{P}\left(\mathbb{R}^{n}\right)$ satisfy $\frac{1}{p_{i}}, \frac{1}{q} \in C^{\log }\left(\mathbb{R}^{n}\right)$ and $s_{i}$ be measurable functions satisfying $s_{i} \in C_{\operatorname{loc}}^{\log }\left(\mathbb{R}^{n}\right) \cap L^{\infty}\left(\mathbb{R}^{n}\right)$, and $\phi$ a set function satisfying the conditions $(\boldsymbol{S 1})$ and $(\boldsymbol{S} 2)$. Under the same assumptions as in Proposition 3.1, the following conclusion

$$
F_{p_{0}(\cdot), q(\cdot)}^{s_{0}(\cdot),}\left(\mathbb{R}^{n}\right) \hookrightarrow F_{p_{1}(\cdot), q(\cdot)}^{s_{1}(\cdot),}\left(\mathbb{R}^{n}\right)
$$

holds true.

Corollary 3.5. Let $i \in\{0,1\}, p_{i}, q_{i} \in \mathcal{P}\left(\mathbb{R}^{n}\right)$ satisfy $\frac{1}{p_{i}}, \frac{1}{q_{i}} \in C^{\log }\left(\mathbb{R}^{n}\right)$ and $s_{i}$ be measurable functions satisfying $s_{i} \in C_{\operatorname{loc}}^{\log }\left(\mathbb{R}^{n}\right) \cap L^{\infty}\left(\mathbb{R}^{n}\right)$, and $\phi$ a set function satisfying the conditions (S1) and (S2). Assume that, for all $x \in \mathbb{R}^{n}$,

$$
s_{0}(x)-\frac{n}{p_{0}(x)}=s_{1}(x)-\frac{n}{p_{1}(x)}
$$

and $\inf _{x \in \mathbb{R}^{n}}\left[s_{0}(x)-s_{1}(x)\right]>0$. Then

$$
F_{p_{0}(\cdot), q_{0}(\cdot)}^{s_{0}(\cdot), \phi}\left(\mathbb{R}^{n}\right) \hookrightarrow F_{p_{1}(\cdot), q_{1}(\cdot)}^{s_{1}(\cdot),}\left(\mathbb{R}^{n}\right) .
$$

Proof. By Proposition 3.2 and (2.6), we see that

$$
f_{p_{0}(\cdot), q_{0}(\cdot)}^{s_{0}(\cdot), \phi}\left(\mathbb{R}^{n}\right) \hookrightarrow f_{p_{0}(\cdot), \infty}^{s_{0}(\cdot), \phi}\left(\mathbb{R}^{n}\right) \hookrightarrow f_{p_{1}(\cdot),\left(q_{1}\right)_{-}}^{s_{1}(\cdot),}\left(\mathbb{R}^{n}\right) \hookrightarrow f_{p_{1}(\cdot), q_{1}(\cdot)}^{s_{1}(\cdot), \phi}\left(\mathbb{R}^{n}\right) .
$$

From this and Theorem 2.3, we deduce that $F_{p_{0}(\cdot), q_{0}(\cdot)}^{s_{0}(\cdot),}\left(\mathbb{R}^{n}\right) \hookrightarrow F_{p_{1}(\cdot), q_{1}(\cdot)}^{s_{1}(\cdot),}\left(\mathbb{R}^{n}\right)$, which completes the proof of Corollary 3.5.

Next we establish molecular and atomic characterizations of Triebel-Lizorkintype spaces with variable exponents.

Definition 3.6. Let $K \in \mathbb{Z}_{+}, L \in \mathbb{Z}$ and $R \in \mathbb{N}$.

(i) A measurable function $m_{Q}$ on $\mathbb{R}^{n}$ is called a $(K, L, R)$-smooth molecule with $Q:=Q_{j k} \in \mathcal{Q}$, where $j \in \mathbb{Z}$ and $k \in \mathbb{Z}^{n}$, if it satisfies the following conditions: 
(M1) (vanishing moment) when $j \in \mathbb{N}, \int_{\mathbb{R}^{n}} x^{\gamma} m_{Q}(x) d x=0$ for all $\gamma \in \mathbb{Z}_{+}^{n}$ and $|\gamma|<L$

(M2) (smoothness condition) for all multi-indices $\alpha \in \mathbb{Z}_{+}^{n}$, with $|\alpha| \leq K$, and all $x \in \mathbb{R}^{n}$

$$
\left|D^{\alpha} m_{Q}(x)\right| \leq 2^{(|\alpha|+n / 2) j}\left(1+2^{j}\left|x-x_{Q}\right|\right)^{-R} .
$$

(ii) A measurable function $a_{Q}$ on $\mathbb{R}^{n}$ is called a $(K, L)$-smooth atom supported near $Q:=Q_{j k} \in \mathcal{Q}$, where $j \in \mathbb{Z}$ and $k \in \mathbb{Z}^{n}$, if it satisfies the following conditions:

(A1) $\operatorname{supp} a_{Q} \subset 3 Q$;

(A2) (vanishing moment) when $j \in \mathbb{N}, \int_{\mathbb{R}^{n}} x^{\gamma} a_{Q}(x) d x=0$ for all $\gamma \in \mathbb{Z}_{+}^{n}$ with $|\gamma|<L$

(A3) (smoothness condition) for all multi-indices $\alpha \in \mathbb{Z}_{+}^{n}$ with $|\alpha| \leq K$,

$$
\left|D^{\alpha} a_{Q}(x)\right| \leq 2^{(|\alpha|+n / 2) j} \text { for all } x \in \mathbb{R}^{n} .
$$

Remark 3.7. (i) If $L<0$, then the vanishing moment conditions (M1) and (A2) are void.

(ii) Let $a_{Q}$ be a $(K, L)$-smooth atom with $Q:=Q_{j k} \in \mathcal{Q}^{*}$ with $j \in \mathbb{Z}_{+}$and $k \in \mathbb{Z}^{n}$. Then, by combining the conditions (A1) and (A3), we conclude that, for all $R \in(0, \infty), \alpha \in \mathbb{Z}_{+}^{n}$ with $|\alpha| \leq K$ and $x \in \mathbb{R}^{n}$,

$$
\left|D^{\alpha} a_{Q}(x)\right| \leq C 2^{(|\alpha|+n / 2) j} \frac{1}{\left(1+2^{j}\left|x-x_{Q}\right|\right)^{R}},
$$

where $C$ is a positive constant independent of $x, \alpha, Q$ and $a_{Q}$, but depending on $R$. Thus, each $(K, L)$-smooth atom is a $(K, L, R)$-smooth molecule up to a harmless positive constant.

Theorem 3.8. Let $p, q, s$ and $\phi$ be as in Definition 1.4.

(i) Let $K \in\left(s_{+}+\max \left\{0, \log _{2} \widetilde{c}_{1}\right\}, \infty\right)$ and

$$
L \in\left(\frac{n}{\min \left\{1, p_{-}, q_{-}\right\}}-n-s_{-}, \infty\right) .
$$

Suppose that $\left\{m_{Q}\right\}_{Q \in \mathcal{Q}^{*}}$ is a family of $(K, L, R)$-smooth molecules with $R$ large enough and that $t:=\left\{t_{Q}\right\}_{Q \in \mathcal{Q}^{*}} \in f_{p(\cdot), q(\cdot)}^{s(\cdot),}\left(\mathbb{R}^{n}\right)$. Then $f:=$ $\sum_{Q \in \mathcal{Q}^{*}} t_{Q} m_{Q}$ converges in $\mathcal{S}^{\prime}\left(\mathbb{R}^{n}\right)$ and

$$
\|f\|_{F_{p(\cdot), q(\cdot)}^{s(\cdot),}\left(\mathbb{R}^{n}\right)} \leq C\|t\|_{f_{p(\cdot), q(\cdot)}^{s(\cdot),}\left(\mathbb{R}^{n}\right)}
$$

with $C$ being a positive constant independent of $t$.

(ii) Conversely, if $f \in F_{p(\cdot), q(\cdot)}^{s(\cdot),}\left(\mathbb{R}^{n}\right)$, then, for any given $K, L \in \mathbb{Z}_{+}$, there exist a sequence $t:=\left\{t_{Q}\right\}_{Q \in \mathcal{Q}^{*}} \subset \mathbb{C}$ and a sequence $\left\{a_{Q}\right\}_{Q \in \mathcal{Q}^{*}}$ of $(K, L)$-smooth atoms such that $f=\sum_{Q \in \mathcal{Q}^{*}} t_{Q} a_{Q}$ in $\mathcal{S}^{\prime}\left(\mathbb{R}^{n}\right)$ and

$$
\|t\|_{f_{p(\cdot), q(\cdot)}^{s(\cdot),}\left(\mathbb{R}^{n}\right)} \leq C\|f\|_{F_{p(\cdot), q(\cdot)}^{s(\cdot), \phi}\left(\mathbb{R}^{n}\right)}
$$

with $C$ being a positive constant independent of $f$. 
Remark 3.9. (i) When $\phi(P):=1$ for all cubes $P \subset \mathbb{R}^{n}$, conclusions of Theorem 3.8 coincide with those of [35, Corollary 5.6]; when $p, q, s$ and $\phi$ are as in Remark 1.5(ii), Theorem 3.8 goes back to [23, Theorem 3.12] (see also [80, Theorem 3.3]).

(ii) In the case that $\phi(P):=1$ for all cubes $P \subset \mathbb{R}^{n}$ and $s \geq 0$, the vanishing moment and the smoothness conditions of Theorem 3.8 can be further refined. Indeed, it was proved in [20, Theorem 3.11] that the vanishing moment and the smoothness conditions of atoms can be localized on dyadic cubes associated with atoms in Theorem 3.8.

Proof of Theorem 3.8. The proof of (ii) is similar to that of [80, Theorme 3.3] (see also [27, Theorem 4.1]). Indeed, by repeating the argument that used in the proof of [80, Theorem 3.3], with [80, Lemma 2.8] therein replaced by Lemma 2.8, we can prove (ii), the details being omitted.

Next we prove (i) by two steps.

Step 1) We show that $f=\sum_{Q \in \mathcal{Q}^{*}} t_{Q} m_{Q}$ converges in $\mathcal{S}^{\prime}\left(\mathbb{R}^{n}\right)$. To this end, it suffices to show that

$$
\lim _{N \rightarrow \infty, \Lambda \rightarrow \infty} \sum_{j=0}^{N} \sum_{k \in \mathbb{Z}^{n},|k| \leq \Lambda} t_{Q_{j k}} m_{Q_{j k}}
$$

exists in $\mathcal{S}^{\prime}\left(\mathbb{R}^{n}\right)$. For all $h \in \mathcal{S}\left(\mathbb{R}^{n}\right)$ and $j \in \mathbb{Z}_{+}$, by the vanishing moment condition (M1), we see that

$$
\begin{aligned}
& \int_{\mathbb{R}^{n}} \sum_{k \in \mathbb{Z}^{n},|k| \leq \Lambda} t_{Q_{j k}} m_{Q_{j k}}(y) h(y) d y \\
& =\int_{\mathbb{R}^{n}} \sum_{k \in \mathbb{Z}^{n},|k| \leq \Lambda} t_{Q_{j k}} m_{Q_{j k}}(y)\left[h(y)-\sum_{\gamma \in \mathbb{Z}^{n},|\gamma|<L}\left(y-x_{Q_{j k}}\right)^{\gamma} \frac{D^{\gamma} h\left(x_{Q_{j k}}\right)}{\gamma !}\right] d y
\end{aligned}
$$

and, by Taylor's remainder theorem, we find that, for all $y \in \mathbb{R}^{n}$,

$$
\begin{aligned}
& \left|h(y)-\sum_{\gamma \in \mathbb{Z}^{n},|\gamma| \leq L}\left(y-x_{Q_{j k}}\right)^{\gamma} \frac{D^{\gamma} h\left(x_{Q_{j k}}\right)}{\gamma !}\right| \\
& \quad \lesssim\left|y-x_{Q_{j k}}\right|^{L} \sum_{|\gamma|=L} \frac{\left|D^{\gamma} h(\xi(y))\right|}{\gamma !} \\
& \quad \lesssim\left(1+\left|y-x_{Q_{j k}}\right|\right)^{L}(1+|y|)^{-\delta} \sup _{\xi \in \mathbb{R}^{n}} \sum_{|\gamma|=L}(1+|\xi|)^{\delta} \frac{\left|D^{\gamma} h(\xi)\right|}{\gamma !},
\end{aligned}
$$

where $\delta \in(0, \infty)$ and $\xi(y):=y+\theta\left(x_{Q_{j k}}-y\right)$ with some $\theta \in(0,1)$ depending on $y$ and $x_{Q_{j k}}$, which, together with the fact that, for all $y \in \mathbb{R}^{n}$,

$$
\left|m_{Q_{j k}}(y)\right| \leq 2^{j n / 2}\left(1+2^{j}\left|y-x_{Q_{j k}}\right|\right)^{-R},
$$

further implies that

$$
\left|\int_{\mathbb{R}^{n}} \sum_{k \in \mathbb{Z}^{n},|k| \leq \Lambda} t_{Q_{j k}} m_{Q_{j k}}(y) h(y) d y\right|
$$




$$
\begin{aligned}
& \lesssim \int_{\mathbb{R}^{n}} \sum_{k \in \mathbb{Z}^{n},|k| \leq \Lambda}\left|t_{Q_{j k}}\right| 2^{-j(L-n / 2)} \frac{(1+|y|)^{-\delta}}{\left(1+2^{j}\left|y-x_{Q_{j k}}\right|\right)^{R-L}} d y \\
& \lesssim \sum_{v=0}^{\infty} \int_{D_{v}} \sum_{k \in \mathbb{Z}^{n}}\left|t_{Q_{j k}}\right| 2^{-j(L-n / 2)} \frac{(1+|y|)^{-\delta}}{\left(1+2^{j}\left|y-x_{Q_{j k}}\right|\right)^{R-L}} d y,
\end{aligned}
$$

where $D_{0}:=\left\{x \in \mathbb{R}^{n}:|x| \leq 1\right\}$ and, for all $v \in \mathbb{N}$,

$$
D_{v}:=\left\{x \in \mathbb{R}^{n}: 2^{v-1}<|x| \leq 2^{v}\right\} .
$$

For all $v \in \mathbb{Z}_{+}, y \in D_{v}$ and $j \in \mathbb{Z}_{+}$, let $W_{0}^{y, j}:=\left\{k \in \mathbb{Z}^{n}: 2^{j}\left|y-x_{Q_{j k}}\right| \leq 1\right\}$ and, for $i \in \mathbb{N}$,

Then we have

$$
W_{i}^{y, j}:=\left\{k \in \mathbb{Z}^{n}: 2^{i-1}<2^{j}\left|y-x_{Q_{j k}}\right| \leq 2^{i}\right\}
$$

$$
\begin{aligned}
H(v, j, y) & :=\sum_{k \in \mathbb{Z}^{n}}\left|t_{Q_{j k}}\right|\left|Q_{j k}\right|^{-\frac{1}{2}}\left(1+2^{j}\left|y-2^{-j} k\right|\right)^{-(R-L)} \\
& \sim \sum_{i=0}^{\infty} \sum_{k \in W_{i}^{y, j}} 2^{-i(R-L)}\left|t_{Q_{j k}}\right|\left|Q_{j k}\right|^{-\frac{1}{2}} \\
& \sim \sum_{i=0}^{\infty} 2^{-i(R-L)} \int_{\cup_{\tilde{k} \in W_{i}^{y, j}} Q_{j \widetilde{k}}} 2^{j n}\left[\sum_{k \in \mathbb{Z}^{n}}\left|t_{Q_{j k}}\right| \widetilde{\chi}_{Q_{j k}}(z)\right] d z .
\end{aligned}
$$

Observe that, if $z \in \cup_{\widetilde{k} \in W_{i}^{y, j}} Q_{j \widetilde{k}}$, then $z \in Q_{j \widetilde{k}_{0}}$ for some $\widetilde{k}_{0} \in W_{i}^{y, j}$ and, for $y \in D_{v}, 1+2^{j}|y-z| \sim 1+2^{i}$; moreover,

$$
|z| \leq\left|z-x_{Q j \widetilde{k}_{0}}\right|+\left|x_{Q j \widetilde{k}_{0}}-y\right|+|y| \lesssim 2^{-j}+2^{-j+i}+2^{v} \lesssim 2^{i+v}
$$

which implies that

$$
\bigcup_{\widetilde{k} \in W_{i}^{y, j}} Q_{j \widetilde{k}} \subset Q\left(0,2^{i+v+c_{0}}\right)
$$

with some positive constant $c_{0} \in \mathbb{N}$. From this, we deduce that, for all $a \in(n, \infty)$, $v, j \in \mathbb{Z}_{+}$and $y \in \mathbb{R}^{n}$,

$$
\begin{aligned}
H(v, j, y) \sim & \sum_{i=0}^{\infty} 2^{-i(R-L-a)} \int_{\cup_{\tilde{k} \in W_{i}^{y, j}} Q_{j \widetilde{k}}} \frac{2^{j n}}{\left(1+2^{j}|y-z|\right)^{a}} \\
& \times\left[\sum_{k \in \mathbb{Z}^{n}}\left|t_{Q_{j k}}\right| \widetilde{\chi}_{Q_{j k}}(z) \chi_{Q\left(0,2^{\left.i+v+c_{0}\right)}\right.}(z)\right] d z \\
\lesssim & \sum_{i=0}^{\infty} 2^{-i(R-L-a)} \eta_{j, a} *\left(\sum_{k \in \mathbb{Z}^{n}}\left|t_{Q_{j k}}\right| \widetilde{\chi}_{Q_{j k}} \chi_{Q\left(0,2^{i+v+c_{0}}\right)}\right)(y),
\end{aligned}
$$

which, combined with (3.3), implies that

$$
\left|\int_{\mathbb{R}^{n}} \sum_{k \in \mathbb{Z}^{n},|k| \leq \Lambda} t_{Q_{j k}} m_{Q_{j k}}(y) h(y) d y\right|
$$




$$
\begin{aligned}
\lesssim & 2^{-j L} \sum_{v=0}^{\infty} \sum_{i=0}^{\infty}\left(1+2^{v}\right)^{-\delta_{0}} 2^{-i(R-L-a)} \\
& \times \int_{D_{v}} \eta_{j, a} *\left(\sum_{k \in \mathbb{Z}^{n}}\left|\widetilde{t}_{Q_{j k}}\right| \chi_{Q_{j k}} \chi_{Q\left(0,2^{i+v+c_{0}}\right)}\right)(y)(1+|y|)^{-\delta+\delta_{0}} d y,
\end{aligned}
$$

where $\delta_{0} \in(0, \infty)$ is determined later.

By (3.1), we find that there exists $r \in\left(0, \min \left\{1, p_{-}, q_{-}\right\}\right)$such that

$$
s_{-}+\frac{n}{p_{-}}(r-1)>-L \text {. }
$$

Let, for all $x \in \mathbb{R}^{n}, \widetilde{p}(x):=p(x) / r,(\widetilde{p}(x))^{*}:=\frac{\widetilde{p}(x)}{\widetilde{p}(x)-1}$ and $\widetilde{s}$ be a measurable function on $\mathbb{R}^{n}$ such that, for all $x \in \mathbb{R}^{n}$,

$$
s(x)-\frac{n}{p(x)}=\widetilde{s}(x)-\frac{n}{\widetilde{p}(x)} .
$$

Then

$$
\begin{aligned}
\widetilde{s}_{-} & =\inf _{x \in \mathbb{R}^{n}}\left\{s(x)+\frac{n(r-1)}{p(x)}\right\} \geq \inf _{x \in \mathbb{R}^{n}}[s(x)]+\inf _{x \in \mathbb{R}^{n}}\left[\frac{n(r-1)}{p(x)}\right] \\
& =s_{-}+\frac{n}{p_{-}}(r-1)>-L .
\end{aligned}
$$

Choosing $\delta \in(0, \infty)$ and $\delta_{0} \in\left(\max \left\{0, \log _{2} \widetilde{c}_{1}\right\}, \infty\right)$ such that

$$
\delta \in\left(n(1-r) / p_{+}+\delta_{0}, \infty\right),
$$

by the Hölder inequality in Remark 1.1(iii), (3.4), Lemma 2.9, Remark 2.2(ii) and Proposition 3.1, we conclude that

$$
\begin{aligned}
& \left|\int_{\mathbb{R}^{n}} \sum_{k \in \mathbb{Z}^{n},|k| \leq \Lambda} t_{Q_{j k}} m_{Q_{j k}}(y) h(y) d y\right| \\
& \lesssim 2^{-j\left(L+\widetilde{s}_{-}\right)} \sum_{v=0}^{\infty} 2^{-v \delta_{0}} \sum_{i=0}^{\infty} 2^{-i(R-L-a)}\left\|(1+|\cdot|)^{-\delta+\delta_{0}}\right\|_{L^{(\widetilde{p}(\cdot))^{*}\left(\mathbb{R}^{n}\right)}} \\
& \times\left\|\eta_{j, a} *\left[\sum_{k \in \mathbb{Z}^{n}} 2^{j \widetilde{s}(\cdot)}\left|t_{Q_{j k}}\right| \widetilde{\chi}_{Q_{j k}} \chi_{Q\left(0,2^{\left.i+v+c_{0}\right)}\right.}\right]\right\|_{L^{\widetilde{p}(\cdot)\left(\mathbb{R}^{n}\right)}} \\
& \lesssim 2^{-j\left(L+\widetilde{s}_{-}\right)} \sum_{v=0}^{\infty} 2^{-v \delta_{0}} \sum_{i=0}^{\infty} 2^{-i(R-L-a)}\left\|\sum_{k \in \mathbb{Z}^{n}} 2^{j \widetilde{s}(\cdot)}\left|t_{Q_{j k}}\right| \widetilde{\chi}_{Q_{j k}}\right\|_{L^{\widetilde{p}(\cdot)}\left(Q\left(0,2^{i+v+c_{0}}\right)\right)} \\
& \lesssim 2^{-j\left(L+\widetilde{s}_{-}\right)} \sum_{v=0}^{\infty} 2^{-v \delta_{0}} \sum_{i=0}^{\infty} 2^{-i(R-L-a)} \phi\left(Q\left(0,2^{i+v+c_{0}}\right)\right)\|t\|_{f_{\widetilde{p}(\cdot), q(\cdot)}^{\widetilde{s}(\cdot)}\left(\mathbb{R}^{n}\right)} \\
& \lesssim 2^{-j\left(L+\widetilde{s}_{-}\right)} \sum_{v=0}^{\infty} 2^{-v\left(\delta_{0}-\log _{2} \widetilde{c}_{1}\right)} \sum_{i=0}^{\infty} 2^{-i\left(R-L-a-\log _{2} \widetilde{c}_{1}\right)}\|t\|_{f_{p(\cdot), q(\cdot)}^{s(\cdot)}\left(\mathbb{R}^{n}\right)} \\
& \lesssim 2^{-j\left(L+\widetilde{s}_{-}\right)}\|t\|_{f_{p(\cdot), q(\cdot)}^{s(\cdot),}\left(\mathbb{R}^{n}\right)},
\end{aligned}
$$


where $R \in(0, \infty)$ is chosen large enough, which, together with $L>-\widetilde{s}_{-}$, implies that (3.2) exists in $\mathcal{S}^{\prime}\left(\mathbb{R}^{n}\right)$ and $|\langle f, h\rangle| \lesssim\|t\|_{f_{p(\cdot), q(\cdot)}^{s(\cdot),\left(\mathbb{R}^{n}\right)}}$.

Step 2) We prove that $\|f\|_{F_{p(\cdot), q(\cdot)}^{s(\cdot),}\left(\mathbb{R}^{n}\right)} \lesssim\|t\|_{f_{p(\cdot), q(\cdot)}^{s(\cdot),}\left(\mathbb{R}^{n}\right)}$. Let $P \in \mathcal{Q}$ be a given dyadic cube and $r \in\left(0, \min \left\{1, p_{-}, q_{-}\right\}\right)$such that $L>n / r-n-s_{-}$. Then, by Remark 1.1(i), we find that

$$
\begin{aligned}
& \frac{1}{\phi(P)}\left\|\left\{\sum_{j=\left(j_{P} \vee 0\right)}^{\infty}\left[2^{j s(\cdot)}\left|\varphi_{j} * f\right|\right]^{q(\cdot)}\right\}^{\frac{1}{q(\cdot)}}\right\|_{L^{p(\cdot)}(P)} \|\left\{\sum^{\infty} \sum_{j=\left(j_{P} \vee 0\right)}^{\infty}\left[2^{j s(\cdot) r} \sum_{v=0}^{\left(j_{P} \vee 0\right)-1} \sum_{\ell(Q)=2^{-v}}\left|t_{Q}\right|^{r}\left|\varphi_{j} * m_{Q}\right|^{r}\right]^{\frac{q(\cdot)}{r}}\left\|_{L^{\frac{p(\cdot)}{r}}(P)}^{\frac{r}{q(\cdot)}}\right\|^{\frac{1}{r}}\right. \\
& \left.\left.\quad \frac{1}{\phi(P)} \| \sum_{v=\left(j_{p} \vee 0\right)} \sum_{\ell(Q)=2^{-v}}^{\infty}\left|t_{Q}\right|^{r}\left|\varphi_{j} * m_{Q}\right|^{r}\right]^{\frac{r}{r}}\right\}_{j=\left(j_{P} \vee 0\right)}^{\frac{r}{q(\cdot)}}\left\|_{2^{j s(\cdot) r}}^{\infty}\right\|_{L^{\frac{p(\cdot)}{r}}(P)} \\
& =: \mathrm{I}_{1}+\mathrm{I}_{2},
\end{aligned}
$$

here $\sum_{v=0}^{\left(j_{P} \vee 0\right)-1} \cdots=0$ if $j_{P} \leq 0$.

Observe that $\mathrm{I}_{1}=0$ if $j_{P} \leq 0$. Thus, to estimate $\mathrm{I}_{1}$, we only need to assume $j_{P} \in \mathbb{N}$. By [26, Lemma 3.3] (see also [35, Lemma 3.5]), we find that, for all $Q:=Q_{v k} \in \mathcal{Q}^{*}$ with $v \leq j$ and $x \in \mathbb{R}^{n}$,

$$
\left|\varphi_{j} * m_{Q}(x)\right| \lesssim 2^{v n / 2} 2^{(v-j) K}\left(1+2^{v}\left|x-x_{Q}\right|\right)^{-R},
$$

which, combined with (2.6), implies that

$$
\begin{aligned}
\mathrm{I}_{1} \lesssim \frac{1}{\phi(P)} \|\left\{\sum_{j=j_{P}}^{\infty} \sum_{v=0}^{j_{P}-1} 2^{j s(\cdot) r} \sum_{k \in \mathbb{Z}^{n}}\left|t_{Q_{v k}}\right|^{r}\left|Q_{v k}\right|^{-\frac{r}{2}}\right. \\
\left.\times 2^{(v-j) K r}\left(1+2^{v}\left|\cdot-x_{Q_{v k}}\right|\right)^{-R r}\right\} \|_{L^{\frac{p(\cdot)}{r}}(P)}^{\frac{1}{r}}
\end{aligned}
$$

We claim that, for all $v, j \in \mathbb{Z}_{+}$and $x \in P$,

$$
\begin{aligned}
J(v, j, x, P):= & 2^{j s(x) r} \sum_{k \in \mathbb{Z}^{n}}\left|t_{Q_{v k}}\right|^{r}\left|Q_{v k}\right|^{-\frac{r}{2}} 2^{(v-j) K r}\left(1+2^{v}\left|x-x_{Q_{v k}}\right|\right)^{-R r} \\
\lesssim & 2^{(v-j)\left(K-s_{+}\right) r} \sum_{i=0}^{\infty} 2^{-i(R-a-\varepsilon / r) r} \\
& \times \eta_{v, a r} *\left(\left[\sum_{k \in \Omega_{i}^{x, v}}\left|t_{Q_{v k}}\right| 2^{v s(\cdot)} \widetilde{\chi}_{Q_{v k}} \chi_{Q\left(c_{P}, 2^{\left.i-v+c_{0}\right)}\right.}\right]^{r}\right)(x),
\end{aligned}
$$


where $a \in(n / r, \infty), \varepsilon \in\left[C_{\log }(s), \infty\right), c_{P}$ is the center of $P, c_{0} \in \mathbb{N}$ is a positive constant independent of $x, P, i, v, k, \Omega_{0}^{x, v}:=\left\{k \in \mathbb{Z}^{n}: 2^{v}\left|x-x_{Q_{v k}}\right| \leq 1\right\}$ and, for all $i \in \mathbb{N}$,

$$
\Omega_{i}^{x, v}:=\left\{k \in \mathbb{Z}^{n}: 2^{i-1}<2^{v}\left|x-x_{Q_{v k}}\right| \leq 2^{i}\right\} .
$$

Indeed, it is easy to see that

$$
\begin{aligned}
& J(v, j, x, P) \lesssim 2^{j s(x) r} 2^{(v-j) K r} \sum_{i=0}^{\infty} \sum_{k \in \Omega_{i}^{x, v}} 2^{-R r i}\left|t_{Q_{v k}}\right|^{r}\left|Q_{v k}\right|^{-\frac{r}{2}} \\
& \sim 2^{[j s(x)+(v-j) K] r} \\
& \times \sum_{i=0}^{\infty}\left\{2^{-R r i+v n} \int_{\cup_{\tilde{k} \in \Omega_{i}^{x}, v} Q_{v \widetilde{k}}}\left[\sum_{k \in \Omega_{i}^{x, v}}\left|t_{Q_{v k}}\right| \widetilde{\chi}_{Q_{v k}}(y)\right]^{r} d y\right\} .
\end{aligned}
$$

Observe that, if $y \in \cup_{\widetilde{k} \in \Omega_{i}^{x, v}} Q_{v \widetilde{k}}$, then there exists a $\widetilde{k}_{0} \in \Omega_{i}^{x, v}$ such that $y \in Q_{v \widetilde{k}_{0}}$ and $1+2^{v}|x-y| \sim 1+2^{i}$; moreover, since $v \leq j_{P}$, it follows that

$$
\begin{aligned}
\left|y-c_{P}\right| & \leq\left|y-x_{Q_{v \widetilde{v}_{0}}}\right|+\left|x-x_{Q_{v \widetilde{k}_{0}}}\right|+\left|x-c_{P}\right| \\
& \lesssim 2^{-v}+2^{i-v}+2^{-j_{P}} \lesssim 2^{i-v},
\end{aligned}
$$

which implies that $\cup_{\widetilde{k} \in \Omega_{i}^{x, v}} Q_{v \widetilde{k}} \subset Q\left(c_{P}, 2^{i-v+c_{0}}\right)$ for some constant $c_{0} \in \mathbb{N}$. From this, (3.6) and Lemma 2.10, we deduce that, for all $a \in(n / r, \infty), v, j \in \mathbb{Z}_{+}$and $x \in P$,

$$
\begin{aligned}
J(v, j, x, P) \lesssim & 2^{j s(x) r} 2^{(v-j) K r} \sum_{i=0}^{\infty} 2^{(a+\varepsilon / r-R) r i} \int_{\cup_{\tilde{k} \in \Omega_{i}^{x, v} Q_{v \widetilde{k}}} \frac{2^{v n}}{\left(1+2^{v}|x-y|\right)^{a r+\varepsilon}}} \\
& \times\left[\sum_{k \in \Omega_{i}^{x, v}}\left|t_{Q_{v k}}\right| \widetilde{\chi}_{Q_{v k}} \chi_{Q\left(c_{P}, 2^{\left.i-v+c_{0}\right)}\right.}(y)\right]^{r} d y \\
& \lesssim 2^{(v-j)\left(K-s_{+}\right) r} \sum_{i=0}^{\infty} 2^{(a+\varepsilon / r-R) r i} \\
& \times \eta_{v, a r} *\left(\left[\sum_{k \in \Omega_{i}^{x, v}} 2^{v s(\cdot)}\left|t_{Q_{v k}}\right| \widetilde{\chi}_{Q_{v k}} \chi_{Q\left(c_{P}, 2^{\left.i-v+c_{0}\right)}\right.}\right]^{r}\right)(x),
\end{aligned}
$$

which implies that the claim holds true.

By this claim, (3.5) and Remark 1.1(i), we conclude that

$$
\begin{aligned}
\mathrm{I}_{1} \lesssim \frac{1}{\phi(P)} & \left\{\sum_{j=j_{P}}^{\infty} \sum_{v=0}^{j_{P}-1} 2^{(v-j)\left(K-s_{+}\right) r} \sum_{i=0}^{\infty} 2^{(a+\varepsilon / r-R) r i}\right. \\
\times & \left.\left\|\eta_{v, a r} *\left(\left[\sum_{k \in \Omega_{i}^{, v}}\left|t_{Q_{v k}}\right| 2^{v s(\cdot)} \tilde{\chi}_{Q_{v k}} \chi_{B\left(c_{P}, 2^{\left.i-v+c_{0}\right)}\right.}\right]^{r}\right)\right\|_{L^{\frac{p(\cdot)}{r}(P)}}\right\}^{\frac{1}{r}},
\end{aligned}
$$


which, together with Lemma 2.9, $j_{P} \in \mathbb{N}$ and Remark 2.2(ii), further implies that

$$
\begin{aligned}
& \mathrm{I}_{1} \lesssim \frac{1}{\phi(P)}\left\{\sum_{j=j_{P}}^{\infty} \sum_{v=0}^{j_{P}} 2^{(v-j)\left(K-s_{+}\right) r} \sum_{i=0}^{\infty} 2^{(a+\varepsilon / r-R) r i}\right. \\
&\left.\quad \times\left\|\sum_{k \in \mathbb{Z}^{n}}\left|t_{Q_{v k}}\right| 2^{v s(\cdot)} \widetilde{\chi}_{Q_{v k}}\right\|_{L^{p(\cdot)}\left(Q \left(c_{P}, 2^{\left.\left.i-v+c_{0}\right)\right)}\right.\right.}^{r}\right\}^{\frac{1}{r}} \\
& \lesssim\|t\|_{f_{p(\cdot), q(\cdot)}^{s(\cdot)}\left(\mathbb{R}^{n}\right)}\left\{\sum_{j=j_{P}}^{\infty} \sum_{v=0}^{j_{P}} 2^{(v-j)\left(K-s_{+}\right) r}\right. \\
&\left.\quad \times \sum_{i=0}^{\infty} 2^{(a+\varepsilon / r-R) r i} \frac{\left[\phi\left(Q\left(c_{P}, 2^{i-v+c_{0}}\right)\right)\right]^{r}}{[\phi(P)]^{r}}\right\}^{\frac{1}{r}} .
\end{aligned}
$$

From this, $K \in\left(s_{+}+\max \left\{0, \log _{2} \widetilde{c}_{1}\right\}, \infty\right)$ and the fact that, when $v \leq j_{P}$,

$$
\begin{aligned}
\phi\left(Q\left(c_{P}, 2^{i-v+c_{0}}\right)\right) & \lesssim\left(\widetilde{c}_{1}\right)^{i} \phi\left(Q\left(c_{P}, 2^{-v}\right)\right) \lesssim\left(\widetilde{c}_{1}\right)^{i+j_{P}-v} \phi(P) \\
& \sim 2^{\left(i+j_{P}-v\right) \log _{2} \widetilde{c}_{1}} \phi(P),
\end{aligned}
$$

we deduce that

$$
\begin{aligned}
\mathrm{I}_{1} \lesssim\|t\|_{f_{p(\cdot), q(\cdot)}^{s(\cdot), \phi}\left(\mathbb{R}^{n}\right)}\left\{2^{j_{P} \log _{2} \widetilde{c}_{1}} \sum_{j=j_{P}}^{\infty} 2^{-j\left(K-s_{+}\right) r} \sum_{v=0}^{j_{P}} 2^{v\left(K-s_{+}-\log _{2} \widetilde{c}_{1}\right) r}\right. \\
\left.\quad \times \sum_{i=0}^{\infty} 2^{-i\left(R-a-\varepsilon / r-\log _{2} \widetilde{c}_{1}\right) r}\right\}^{\frac{1}{r}} \lesssim\|t\|_{f_{p(\cdot), q(\cdot)}^{s(\cdot),}\left(\mathbb{R}^{n}\right)},
\end{aligned}
$$

where $R \in(0, \infty)$ is chosen such that $R>a+\varepsilon / r+\log _{2} \widetilde{c}_{1}$.

We now estimate $\mathrm{I}_{2}$. By applying [20, Lemmas A.2 and A.5] and an argument similar to that used in the proof of [20, Lemma 6.3], we see that, for all $j \in \mathbb{Z}_{+}$, $Q:=Q_{v k} \in \mathcal{Q}^{*}$ and $x \in \mathbb{R}^{n}$,

$$
\left|\varphi_{j} * m_{Q}(x)\right| \lesssim 2^{-\beta(j, v)}|Q|^{-1 / 2}\left(\eta_{j, R} * \eta_{v, R} * \chi_{Q}\right)(x)
$$

where

$$
\beta(j, v):=K \max \{j-v, 0\}+L \max \{v-j, 0\}
$$

Let

$$
M \in\left(n / r+\log _{2}\left(c_{1} \widetilde{c}_{1}\right), \infty\right)
$$

and $\varepsilon \in\left[C_{\log }(s), \infty\right)$ be such that $R=2 M+\varepsilon / r$. Thus, we have

$$
\begin{aligned}
& \mathrm{I}_{2} \lesssim \frac{1}{\phi(P)} \|\left\{\sum _ { j = ( j _ { P } \vee 0 ) } ^ { \infty } \left[\sum_{v=\left(j_{P} \vee 0\right)}^{\infty} \sum_{\ell(Q)=2^{-v}} 2^{j s(\cdot) r} 2^{-\beta(j, v) r}\right.\right. \\
& \left.\left.\quad \times\left|t_{Q}\right|^{r}|Q|^{-\frac{r}{2}}\left(\eta_{j, 2 M+\varepsilon / r} * \eta_{v, 2 M+\varepsilon / r} * \chi_{Q}\right)^{r}\right]^{\frac{q(\cdot)}{r}}\right\}^{\frac{r}{q(\cdot)}} \|_{L^{p(\cdot)}(P)}^{\frac{1}{r}} .
\end{aligned}
$$


By [20, Lemma A.4], we find that, for all $v \in \mathbb{Z}_{+}$and $\ell(Q)=2^{-v}$,

$$
\begin{aligned}
& 2^{j s(\cdot) r-\beta(j, v) r}\left(\eta_{j, 2 M+\varepsilon / r} * \eta_{v, 2 M+\varepsilon / r} * \chi_{Q}\right)^{r} \\
& \lesssim 2^{j s(\cdot) r-\beta(j, v) r} 2^{n \max \{v-j, 0\}(1-r)} \eta_{j, 2 M r+\varepsilon} * \eta_{v, 2 M r+\varepsilon} * \chi_{Q} \\
& \sim 2^{v s(\cdot) r} 2^{-\left(K-s_{+}\right) r \max \{j-v, 0\}} \\
& \quad \times 2^{-\left(L-\frac{n}{r}+n+s_{-}\right) r \max \{v-j, 0\}} \eta_{j, 2 M r+\varepsilon} * \eta_{v, 2 M r+\varepsilon} * \chi_{Q},
\end{aligned}
$$

which, combined with (3.8) and Lemma 2.10, implies that

$$
\begin{aligned}
& \mathrm{I}_{2} \lesssim \frac{1}{\phi(P)} \|\left\{\sum _ { j = ( j _ { P } \vee 0 ) } ^ { \infty } \left(\eta _ { j , 2 M r } * \left[\sum_{v=\left(j_{P} \vee 0\right)}^{\infty} \sum_{\ell(Q)=2^{-v}}\left|t_{Q}\right|^{r}|Q|^{-\frac{r}{2}}\right.\right.\right. \\
& \left.\left.\left.\quad \times 2^{v s(\cdot) r-\varepsilon(j, v) r} \eta_{v, 2 M r+\varepsilon} * \chi_{Q}\right]\right)^{\frac{q(\cdot)}{r}}\right\}^{\frac{r}{q(\cdot)}} \|_{L^{\frac{p(\cdot)}{r}}(P)}^{\frac{1}{r}} \\
& \lesssim \frac{1}{\phi(P)} \|\left\{\sum _ { j = ( j _ { P } \vee 0 ) } ^ { \infty } \left[\sum_{l \in \mathbb{Z}^{n}} \int_{P+l \ell(P)} \frac{2^{j n}}{\left(1+2^{j}|\cdot-y|\right)^{2 M r}} \sum_{v=\left(j_{P} \vee 0\right)}^{\infty} 2^{v s(\cdot)-\varepsilon(j, v)}\right.\right. \\
& \left.\left.\quad \times \sum_{\ell(Q)=2^{-v}}\left[\frac{\left|t_{Q}\right|}{|Q|^{\frac{1}{2}}}\right]^{r} \eta_{v, 2 M r+\varepsilon} * \chi_{Q}\right)(y) d y\right]^{\frac{q(\cdot)}{r}} \|_{L^{\frac{p(\cdot)}{r}(P)}}^{\frac{1}{r}}
\end{aligned}
$$

where

$$
\varepsilon(j, v):=\left(K-s_{+}\right) \max \{j-v, 0\}+\left(L-n / r+n+s_{-}\right) \max \{v-j, 0\} .
$$

From this, the fact that, when $j \geq j_{P}, l \in \mathbb{Z}^{n}, x \in P$ and $y \in P+l \ell(P)$,

$$
1+2^{j}|x-y| \geq 1+2^{j_{P}}|x-y| \sim 1+|l|
$$

the Minkowski inequality, Lemma 2.9 and Remark 1.1(i), we further deduce that

$$
\begin{aligned}
& \mathrm{I}_{2} \lesssim \frac{1}{\phi(P)} \|\left\{\sum _ { j = ( j _ { P } \vee 0 ) } ^ { \infty } \left[\sum _ { l \in \mathbb { Z } ^ { n } } ( 1 + | l | ) ^ { - M r } \eta _ { j , M r } * \left(\left[\sum_{v=\left(j_{P} \vee 0\right)}^{\infty} \sum_{\ell(Q)=2^{-v}}\left|t_{Q}\right|^{r}\right.\right.\right.\right. \\
& \left.\left.\left.\left.\quad \times|Q|^{-\frac{r}{2}} 2^{v s(\cdot) r-\varepsilon(j, v) r} \eta_{v, 2 M r+\varepsilon} * \chi_{Q}\right] \chi_{P+l \ell(P)}\right)\right]^{\frac{q(\cdot)}{r}}\right\}^{\frac{r}{q(\cdot)}} \|_{L^{\frac{p}{r}}}^{\frac{p(\cdot)}{r}}(P) \\
& \lesssim \frac{1}{\phi(P)} \| \sum_{l \in \mathbb{Z}^{n}}(1+|l|)^{-M r}\left\{\sum _ { j = ( j _ { P } \vee 0 ) } ^ { \infty } \left[\eta _ { j , M r } * \left(\left[\sum_{v=\left(j_{P} \vee 0\right)}^{\infty} \sum_{\ell(Q)=2^{-v}}\left|t_{Q}\right|^{r}\right.\right.\right.\right.
\end{aligned}
$$




$$
\begin{aligned}
& \left.\left.\left.\left.\times|Q|^{-\frac{r}{2}} 2^{v s(\cdot) r-\varepsilon(j, v) r} \eta_{v, 2 M r+\varepsilon} * \chi_{Q}\right] \chi_{P+l \ell(P)}\right)\right]^{\frac{q(\cdot)}{r}}\right\}^{\frac{r}{q(\cdot)}} \|_{L^{\frac{p(\cdot)}{r}(P)}}^{\frac{1}{r}} \\
\lesssim \frac{1}{\phi(P)} & \left\{\sum_{l \in \mathbb{Z}^{n}}(1+|l|)^{-M r} \|\left[\sum _ { j = ( j _ { P } \vee 0 ) } ^ { \infty } \left(\sum_{v=\left(j_{P} \vee 0\right)}^{\infty} \sum_{\ell(Q)=2^{-v}}\left|t_{Q}\right|^{r}|Q|^{-\frac{r}{2}}\right.\right.\right. \\
& \left.\left.\left.\times 2^{v s(\cdot) r-\varepsilon(j, v) r} \eta_{v, 2 M r+\varepsilon} * \chi_{Q}\right)^{\frac{q(\cdot)}{r}}\right]^{\frac{r}{q(\cdot)}}\right\}_{L^{\frac{p(\cdot)}{r}}(P+l \ell(P))}
\end{aligned}
$$

By the Hölder inequality,

$$
K \in\left(s_{+}+\max \left\{0, \log _{2} \widetilde{c}_{1}\right\}, \infty\right),
$$

$L \in\left(n / r-n-s_{-}, \infty\right)$ and the fact that $0<q_{-} \leq q_{+}<\infty$, we see that

$$
\begin{aligned}
& {\left[\sum_{j=\left(j_{P} \vee 0\right)}^{\infty}\left(\sum_{v=\left(j_{P} \vee 0\right)}^{\infty} \sum_{\ell(Q)=2^{-v}}\left|t_{Q}\right|^{r}|Q|^{-\frac{r}{2}} 2^{v s(\cdot) r-\varepsilon(j, v) r} \eta_{v, 2 M r+\varepsilon} * \chi_{Q}\right)^{\frac{q(\cdot)}{r}}\right]^{\frac{r}{q(\cdot)}}} \\
& \lesssim\left\{\sum_{j=\left(j_{P} \vee 0\right)}^{\infty} \sum_{v=\left(j_{P} \vee 0\right)}^{\infty} 2^{-\varepsilon(j, v) r}\left[\sum_{\ell(Q)=2^{-v}}\left|t_{Q}\right|^{r}|Q|^{-\frac{r}{2}} 2^{v s(\cdot) r} \eta_{v, 2 M r+\varepsilon} * \chi_{Q}\right]^{\frac{q(\cdot)}{r}}\right\}^{\frac{r}{q(\cdot)}} \\
& \lesssim\left\{\sum_{v=\left(j_{P} \vee 0\right)}^{\infty}\left[\sum_{\ell(Q)=2^{-v}}\left|t_{Q}\right|^{r}|Q|^{-\frac{r}{2}} 2^{v s(\cdot) r} \eta_{v, 2 M r+\varepsilon} * \chi_{Q}\right]^{\frac{q(\cdot)}{r}}\right\}^{\frac{r}{q(\cdot)}},
\end{aligned}
$$

which, together with (3.10) and some arguments similar to those used in the proofs of (3.9) and (3.10), implies that

$$
\begin{aligned}
\mathrm{I}_{2} \lesssim \frac{1}{\phi(P)}\left[\sum_{l \in \mathbb{Z}^{n}}(1+|l|)^{-M r} \| \sum_{v=\left(j_{P} \vee 0\right)}^{\infty}\left[\sum_{\ell(Q)=2^{-v}}\left|t_{Q}\right|^{r}|Q|^{-\frac{r}{2}}\right.\right. \\
\left.\left.\left.\left.\left.\quad \times 2^{v s(\cdot) r} \eta_{v, 2 M r+\varepsilon} * \chi_{Q}\right]^{\frac{q(\cdot)}{r}}\right\}\right]^{\frac{1}{r}}\right]_{L^{\frac{p(\cdot)}{r}}(P+l \ell(P))}\right] \\
\lesssim \frac{1}{\phi(P)}\left\{\sum_{l \in \mathbb{Z}^{n}}(1+|l|)^{-M r} \|\left(\sum _ { v = ( j _ { P } \vee 0 ) } ^ { \infty } \left[\sum_{k \in \mathbb{Z}^{n}}(1+|k|)^{-M r}\right.\right.\right.
\end{aligned}
$$




$$
\begin{aligned}
& \times \eta_{v, M r} *\left(\left\{\sum_{\ell(Q)=2^{-v}}\left|t_{Q}\right| 2^{v s(\cdot)} \tilde{\chi}_{Q}\right\}^{r}\right. \\
& \left.\left.\left.\left.\times \chi_{P+(l+k) \ell(P)}\right)\right]^{\frac{q(\cdot)}{r}}\right)^{\frac{r}{q(\cdot)} \|}\right\}_{L^{\frac{p(\cdot)}{r}}(P+l \ell(P))}^{\frac{1}{r}} .
\end{aligned}
$$

From this, the Minkowski inequality, Remark 1.1(i), Lemmas 2.9 and 2.6(ii), we deduce that

$$
\begin{aligned}
\mathrm{I}_{2} & \lesssim \frac{1}{\phi(P)}\left[\sum_{k, l \in \mathbb{Z}^{n}}(1+|l|)^{-M r}(1+|k|)^{-M r}\right. \\
& \left.\times \|\left.\left\{\sum_{v=\left(j_{P} \vee 0\right)}^{\infty}\left[\sum_{\ell(Q)=2^{-v}} 2^{v s(\cdot)}\left|t_{Q}\right| \widetilde{\chi}_{Q}\right]^{q(\cdot)}\right\}^{\frac{1}{q(\cdot)} \|^{r}}\right|_{L^{p(\cdot)}(P+(l+k) \ell(P))} ^{r}\right]^{\frac{1}{r}} \\
& \lesssim\|t\|_{f_{p(\cdot), q(\cdot)}^{s(\cdot),}\left(\mathbb{R}^{n}\right)}\left\{\sum_{k, l \in \mathbb{Z}^{n}}(1+|l|)^{-M r}(1+|k|)^{-M r} \frac{[\phi(P+(l+k) \ell(P))]^{r}}{[\phi(P)]^{r}}\right\}^{\frac{1}{r}} \\
& \lesssim\|t\|_{f_{p(\cdot), q(\cdot)}^{s(\cdot),}\left(\mathbb{R}^{n}\right)}\left\{\sum_{k, l \in \mathbb{Z}^{n}}(1+|l|)^{-M r+r \log _{2}\left(c_{1} \widetilde{c}_{1}\right)}(1+|k|)^{-M r+r \log _{2}\left(c_{1} \widetilde{c}_{1}\right)}\right\}^{\frac{1}{2}} \\
& \sim\|t\|_{f_{p(\cdot), q(\cdot)}^{s(\cdot),}\left(\mathbb{R}^{n}\right)},
\end{aligned}
$$

where $M$ is chosen large enough.

Finally, combining (3.7) and (3.11), we conclude that

$$
\|f\|_{F_{p(\cdot), q(\cdot)}^{s(\cdot),}\left(\mathbb{R}^{n}\right)} \lesssim \sup _{P \in \mathcal{Q}}\left(\mathrm{I}_{1}+\mathrm{I}_{2}\right) \lesssim\|t\|_{f_{p(\cdot), q(\cdot)}^{s(\cdot),}\left(\mathbb{R}^{n}\right)},
$$

which completes the proof of Theorem 3.8.

Next we establish the Peetre maximal function characterization of $F_{p(\cdot), q(\cdot)}^{s(\cdot),}\left(\mathbb{R}^{n}\right)$.

Let $(\varphi, \Phi)$ be a pair of admissible functions. Recall that the Peetre maximal function of $f \in \mathcal{S}^{\prime}\left(\mathbb{R}^{n}\right)$ is defined by setting, for all $j \in \mathbb{Z}_{+}, a \in(0, \infty)$ and $x \in \mathbb{R}^{n}$,

$$
\left(\varphi_{j}^{*} f\right)_{a}(x):=\sup _{y \in \mathbb{R}^{n}} \frac{\left|\varphi_{j} * f(x+y)\right|}{\left(1+2^{j}|y|\right)^{a}}
$$

where $\varphi_{0}$ is replaced by $\Phi$. The following Lemma 3.10 comes from [71, (2.48) and (2.66)].

Lemma 3.10. Let $(\varphi, \Phi)$ be a pair of admissible functions, $f \in \mathcal{S}^{\prime}\left(\mathbb{R}^{n}\right)$ and $N \in \mathbb{N}$. Then, for all $t \in[1,2], a \in(0, N], \ell \in \mathbb{Z}_{+}$and $x \in \mathbb{R}^{n}$,

$$
\left[\left(\varphi_{2^{-\ell} t}^{*} f\right)_{a}(x)\right]^{r} \leq C \sum_{v=0}^{\infty} 2^{-v N r} 2^{(v+\ell) n} \int_{\mathbb{R}^{n}} \frac{\left|\left(\varphi_{v+\ell}\right)_{t} * f(y)\right|^{r}}{\left(1+2^{\ell}|x-y|\right)^{a r}} d y
$$


where $r$ is an arbitrary fixed positive number, $\varphi_{0}$ is replaced by $\Phi$ and $C$ is a positive constant independent of $\varphi, \Phi, f, x, \ell$ and $t$.

Theorem 3.11. Let $p, q, s$ and $\phi$ be as in Definition 1.4. Let

$$
a \in\left(\frac{n}{\min \left\{p_{-}, q_{-}\right\}}+\log _{2} \widetilde{c}_{1}+C_{\log }(s), \infty\right) .
$$

Then $f \in F_{p(\cdot), q(\cdot)}^{s(\cdot), \phi}\left(\mathbb{R}^{n}\right)$ if and only if $f \in \mathcal{S}^{\prime}\left(\mathbb{R}^{n}\right)$ and $\|f\|_{F_{p(\cdot), q(\cdot)}^{s(\cdot),}\left(\mathbb{R}^{n}\right)}^{*}<\infty$, where

$$
\|f\|_{F_{p(\cdot), q(\cdot)}^{s(\cdot),\left(\mathbb{R}^{n}\right)}}^{*}:=\sup _{P \in \mathcal{Q}} \frac{1}{\phi(P)}\left\|\left\{\sum_{j=\left(j_{P} \vee 0\right)}^{\infty}\left[2^{j s(\cdot)}\left(\varphi_{j}^{*} f\right)_{a}\right]^{q(\cdot)}\right\}^{\frac{1}{q(\cdot)}}\right\|_{L^{p(\cdot)}(P)} .
$$

Proof. Observe that, by definitions, we have $\|f\|_{F_{p(\cdot), q(\cdot)}^{s(\cdot),}\left(\mathbb{R}^{n}\right)} \leq\|f\|_{F_{p(\cdot), q(\cdot)}^{s(\cdot),\left(\mathbb{R}^{n}\right)}}^{*}$. Next we show that $\|f\|_{F_{p(\cdot), q(\cdot)}^{s(\cdot) \phi}\left(\mathbb{R}^{n}\right)}^{*} \lesssim\|f\|_{F_{p(\cdot), q(\cdot)}^{s(\cdot), \phi}\left(\mathbb{R}^{n}\right)}$ for all $f \in F_{p(\cdot), q(\cdot)}^{s(\cdot), \phi}\left(\mathbb{R}^{n}\right)$.

By (3.12), we find that there exist $r \in\left(0, \min \left\{p_{-}, q_{-}\right\}\right)$and $\varepsilon \in\left(\log _{2} \widetilde{c}_{1}, \infty\right)$ such that $a>n / r+\varepsilon+C_{\log }(s)$. For any given dyadic cube $P \subset \mathbb{R}^{n}$, by Lemma 3.10 , we see that

$$
\begin{aligned}
\mathrm{J}_{P}:= & \frac{1}{\phi(P)}\left\|\left\{\sum_{j=\left(j_{P} \vee 0\right)}^{\infty}\left[2^{j s(\cdot)}\left(\varphi_{j}^{*} f\right)_{a}\right]^{q(\cdot)}\right\}^{\frac{1}{q(\cdot)}}\right\|_{L^{p(\cdot)}(P)} \\
\lesssim & \frac{1}{\phi(P)} \|\left\{\sum _ { j = ( j _ { P } \vee 0 ) } ^ { \infty } \left[2 ^ { j s ( \cdot ) } \left(\sum_{v=0}^{\infty} 2^{-v N r} 2^{(v+j) n}\right.\right.\right. \\
& \left.\left.\left.\times \int_{\mathbb{R}^{n}} \frac{\left|\varphi_{v+j} * f(y)\right|^{r}}{\left(1+2^{j}|\cdot-y|\right)^{a r}} d y\right)^{\frac{1}{r}}\right]^{q(\cdot)}\right\}^{\frac{1}{q(\cdot)}} \|_{L^{p(\cdot)(P)}},
\end{aligned}
$$

where $N \in \mathbb{N} \cap[a, \infty)$ is determined later. Notice that $j \geq\left(j_{P} \vee 0\right)$ and, for all $x \in P$ and $y \in\left(2^{k+1} \sqrt{n} P\right) \backslash\left(2^{k} \sqrt{n} P\right)=: D_{k, P}$ with $k \in \mathbb{N}$,

$$
1+2^{j}|x-y| \gtrsim 2^{j} 2^{-j_{P}} 2^{k}
$$

Then it follows that, for all $x \in P$,

$$
\begin{aligned}
& \int_{\mathbb{R}^{n}} \frac{\left|\varphi_{v+j} * f(y)\right|^{r}}{\left(1+2^{j}|x-y|\right)^{a r}} d y \\
& =\left\{\int_{2 \sqrt{n} P}+\sum_{k=1}^{\infty} \int_{D_{k, P}}\right\} \frac{\left|\varphi_{v+j} * f(y)\right|^{r}}{\left(1+2^{j}|x-y|\right)^{a r}} d y \\
& \lesssim 2^{-j n} \eta_{j, a r} *\left(\left|\varphi_{v+j} * f\right|^{r} \chi_{2 \sqrt{n} P}\right)(x) \\
& \quad+2^{-j(\varepsilon r+n)} 2^{j p \varepsilon r} \sum_{k=1}^{\infty} 2^{-k \varepsilon r} \eta_{j,(a-\varepsilon) r} *\left(\left|\varphi_{v+j} * f\right|^{r} \chi_{D_{k, P}}\right)(x)
\end{aligned}
$$




$$
=: \mathrm{I}_{P, 1}+\mathrm{I}_{P, 2},
$$

which implies that

$$
\begin{aligned}
\mathrm{J}_{P} \lesssim \frac{1}{\phi(P)}\left\|\left\{\sum_{j=\left(j_{P} \vee 0\right)}^{\infty}\left[2^{j s(\cdot) r} \sum_{v=0}^{\infty} 2^{-v N r} 2^{(v+j) n} \mathrm{I}_{P, 1}\right]^{\frac{q(\cdot)}{r}}\right\}^{\frac{1}{q(\cdot)}}\right\|_{L^{p(\cdot)}(P)} \\
\quad+\frac{1}{\phi(P)}\left\|\left\{\sum_{j=\left(j_{P} \vee 0\right)}^{\infty}\left[2^{j s(\cdot) r} \sum_{v=0}^{\infty} 2^{-v N r} 2^{(v+j) n} \mathrm{I}_{P, 2}\right]^{\frac{q(\cdot)}{r}}\right\}^{\frac{1}{q(\cdot)}}\right\|_{L^{p(\cdot)}(P)} \\
=: \mathrm{J}_{P, 1}+\mathrm{J}_{P, 2} .
\end{aligned}
$$

For $\mathrm{J}_{P, 1}$, by Lemmas 2.10 and 2.9, the Minkowski inequality and Remark 1.1(i), we find that

$$
\begin{aligned}
\mathrm{J}_{P, 1} & \lesssim \frac{1}{\phi(P)}\left\|\left\{\sum_{j=\left(j_{P} \vee 0\right)}^{\infty}\left[\sum_{v=0}^{\infty} 2^{(n-N r) v} 2^{j s(\cdot) r}\left|\varphi_{v+j} * f\right|^{r}\right]^{\frac{q(\cdot)}{r}}\right\}^{\frac{r}{q \cdot \cdot)}}\right\|_{L^{\frac{p(\cdot)}{r}}(2 \sqrt{n} P)}^{\frac{1}{r}} \\
\lesssim & \left\{\sum_{v=0}^{\infty} 2^{-v(N r-n+s-r)} \frac{1}{[\phi(P)]^{r}}\right. \\
& \times\left\|\left\{\sum_{j=\left(j_{p} \vee 0\right)}^{\infty}\left[2^{(v+j) s(\cdot)}\left|\varphi_{v+j} * f\right|^{r}\right]^{q(\cdot)}\right\}^{\frac{1}{q(\cdot)}}\right\|^{r} \|_{L^{p(\cdot)}(2 \sqrt{n} P)}^{\frac{1}{r}} \\
\lesssim & \left\{\sum_{v=0}^{\infty} 2^{-v\left(N r-n+s_{-} r\right)}\right\}^{\frac{1}{r}}\|f\|_{F_{p(\cdot), q(\cdot)}^{s(\cdot), \phi}\left(\mathbb{R}^{n}\right)} \sim\|f\|_{F_{p(\cdot), q(\cdot)}^{s(\cdot),\left(\mathbb{R}^{n}\right)},}
\end{aligned}
$$

where we used the condition (S1) of $\phi$ in the third inequality and $N \in \mathbb{N}$ is chosen large enough such that $N \in[a, \infty) \cap\left(\frac{n}{r}-s_{-}, \infty\right)$.

For $\mathrm{J}_{P, 2}$, by an argument similar to the above, we find that

$$
\begin{aligned}
\mathrm{J}_{P, 2} \lesssim & \left\{\sum_{v=0}^{\infty} 2^{-v\left(N r-n+r s_{-}\right)} \sum_{k=1}^{\infty} 2^{-k \varepsilon} \frac{1}{[\phi(P)]^{r}}\right. \\
& \left.\times\left\|\left\{\sum_{j=\left(j_{p} \vee 0\right)}^{\infty}\left[2^{(v+j) s(\cdot)}\left|\varphi_{v+j} * f\right|^{r}\right]^{q(\cdot)}\right\}^{\frac{1}{q(\cdot)}}\right\|^{r} \|_{L^{p(\cdot)}\left(D_{k, P}\right)}^{r}\right\}^{\frac{1}{r}} \\
\lesssim & \left\{\sum_{v=0}^{\infty} 2^{-v\left(N r-n+r s_{-}\right)} \sum_{k=1}^{\infty} 2^{-k \varepsilon r} \frac{\left[\phi\left(2^{k+1+n} P\right)\right]^{r}}{[\phi(P)]^{r}}\right\}^{\frac{1}{r}}\|f\|_{F_{p(\cdot), q(\cdot)}^{s(\cdot),\left(\mathbb{R}^{n}\right)}}
\end{aligned}
$$




$$
\lesssim\left\{\sum_{k=1}^{\infty} 2^{-k\left(\varepsilon-\log _{2} \widetilde{c}_{1}\right)}\right\}^{\frac{1}{r}}\|f\|_{F_{p(\cdot), q(\cdot)}^{s(\cdot),}\left(\mathbb{R}^{n}\right)} \sim\|f\|_{F_{p(\cdot), q(\cdot)}^{s(\cdot),}\left(\mathbb{R}^{n}\right)} .
$$

Combining the estimates (3.13), (3.14), (3.15) and (3.16), we conclude that

$$
\|f\|_{F_{p(\cdot), q(\cdot)}^{s(\cdot),}\left(\mathbb{R}^{n}\right)}^{*} \leq \sup _{P \in \mathcal{Q}} \mathrm{J}_{P} \lesssim \sup _{P \in \mathcal{Q}}\left(\mathrm{J}_{P, 1}+\mathrm{J}_{P, 2}\right) \lesssim\|f\|_{F_{p(\cdot), q(\cdot)}^{s(\cdot), \phi}\left(\mathbb{R}^{n}\right)},
$$

which completes the proof of Theorem 3.11.

As applications of Theorem 3.11, we obtain two equivalent quasi-norms of the space $F_{p(\cdot), q(\cdot)}^{s(\cdot),}\left(\mathbb{R}^{n}\right)$. To this end, for all $f \in \mathcal{S}^{\prime}\left(\mathbb{R}^{n}\right)$, let

$$
\left\|f \mid F_{p(\cdot), q(\cdot)}^{s(\cdot),}\left(\mathbb{R}^{n}\right)\right\|_{1}:=\sup _{P \in \mathcal{Q}} \frac{1}{\phi(P)}\left\|\left\{\sum_{j=0}^{\infty}\left[2^{j s(\cdot)}\left|\varphi_{j} * f\right|\right]^{q(\cdot)}\right\}^{\frac{1}{q(\cdot)}}\right\|_{L^{p(\cdot)}(P)}
$$

and

$$
\left\|f\left|F_{p(\cdot), q(\cdot)}^{s(\cdot), \phi}\left(\mathbb{R}^{n}\right)\left\|_{2}:=\sup _{Q \in \mathcal{Q}} \sup _{x \in Q}|Q|^{-\frac{s(x)}{n}}[\phi(Q)]^{-1}\right\| \chi_{Q} \|_{L^{p(\cdot)}\left(\mathbb{R}^{n}\right)}\right| \varphi_{j_{Q}} * f(x) \mid .\right.
$$

Theorem 3.12. Let $p, q, s, \phi$ be as in Definition 1.4.

(i) If $\widetilde{c}_{1} \in\left(0,2^{n / p_{+}}\right)$, then $f \in F_{p(\cdot), q(\cdot)}^{s(\cdot),}\left(\mathbb{R}^{n}\right)$ if and only if $f \in \mathcal{S}^{\prime}\left(\mathbb{R}^{n}\right)$ and $\left\|f \mid F_{p(\cdot), q(\cdot)}^{s(\cdot),}\left(\mathbb{R}^{n}\right)\right\|_{1}<\infty$; moreover, there exists a positive constant $C$, independent of $f$, such that

$$
C^{-1}\|f\|_{F_{p(\cdot), q(\cdot)}^{s(\cdot),}\left(\mathbb{R}^{n}\right)} \leq\left\|f \mid F_{p(\cdot), q(\cdot)}^{s(\cdot), \phi}\left(\mathbb{R}^{n}\right)\right\|_{1} \leq C\|f\|_{F_{p(\cdot), q(\cdot)}^{s(\cdot),}\left(\mathbb{R}^{n}\right)} .
$$

(ii) If $c_{1} \in\left(0,2^{-n / p_{-}}\right)$, then $f \in F_{p(\cdot), q(\cdot)}^{s(\cdot),}\left(\mathbb{R}^{n}\right)$ if and only if $f \in \mathcal{S}^{\prime}\left(\mathbb{R}^{n}\right)$ and $\left\|f \mid F_{p(\cdot), q(\cdot)}^{s(\cdot),}\left(\mathbb{R}^{n}\right)\right\|_{2}<\infty$; moreover, there exists a positive constant $C$, independent of $f$, such that

$$
C^{-1}\|f\|_{F_{p(\cdot), q(\cdot)}^{s(\cdot),}\left(\mathbb{R}^{n}\right)} \leq\left\|f \mid F_{p(\cdot), q(\cdot)}^{s(\cdot), \phi}\left(\mathbb{R}^{n}\right)\right\|_{2} \leq C\|f\|_{F_{p(\cdot), q(\cdot)}^{s(\cdot),}\left(\mathbb{R}^{n}\right)} .
$$

Proof. We first show (i). To this end, it suffices to show that

$$
\left\|f \mid F_{p(\cdot), q(\cdot)}^{s(\cdot), \phi}\left(\mathbb{R}^{n}\right)\right\|_{1} \lesssim\|f\|_{F_{p(\cdot), q(\cdot)}^{s(\cdot)}\left(\mathbb{R}^{n}\right)}
$$

since the inverse inequality obviously holds true by definitions.

Let $P \in \mathcal{Q}$ be a given dyadic cube. By Remark 1.1(i), we see that

$$
\begin{aligned}
\mathrm{I}_{P} & :=\frac{1}{\phi(P)}\left\|\left\{\sum_{j=0}^{\infty}\left[2^{j s(\cdot)}\left|\varphi_{j} * f\right|\right]^{q(\cdot)}\right\}^{\frac{1}{q(\cdot)}}\right\|_{L^{p(\cdot)}(P)} \\
& \lesssim \frac{1}{\phi(P)} \|\left\{\sum_{j=0}^{\frac{1}{q(\cdot)}}\left\|_{\left.2^{j s(\cdot)}\left|\varphi_{j} * f\right|\right]^{q(\cdot)}}\right\|_{L^{p(\cdot)}(P)}\right.
\end{aligned}
$$




$$
+\frac{1}{\phi(P)}\left\|\left\{\sum_{j=\left(j_{P} \vee 0\right)}^{\infty}\left[2^{j s(\cdot)}\left|\varphi_{j} * f\right|\right]^{q(\cdot)}\right\}^{\frac{1}{q(\cdot)}}\right\|_{L^{p(\cdot)}(P)}=: \mathrm{I}_{P, 1}+\mathrm{I}_{P, 2}
$$

where $\sum_{j=0}^{\left(j_{P} \vee 0-1\right)} \cdots=0$ if $j_{P} \leq 0$.

Obviously, $\mathrm{I}_{P, 2} \leq\|f\|_{F_{p(\cdot), q(\cdot)}^{s(\cdot),}\left(\mathbb{R}^{n}\right)}$.

For $\mathrm{I}_{P, 1}$, we only need to estimate it in the case that $j_{P}>0$. For any $j \in \mathbb{N}$ with $j \leq j_{P}-1$, there exists a unique dyadic cube $P_{j}$ such that $P \subset P_{j}$ and $\ell\left(P_{j}\right)=2^{-j}$. Since $s \in C_{\text {loc }}^{\log }\left(\mathbb{R}^{n}\right) \cap L^{\infty}\left(\mathbb{R}^{n}\right)$, it follows that, for all $a \in(0, \infty)$, $x \in P$ and $y \in P_{j}$,

$$
\begin{aligned}
2^{j s(x)}\left|\varphi_{j} * f(x)\right| & \lesssim 2^{j s(x)}\left(1+2^{j}|x-y|\right)^{a}\left(\varphi_{j}^{*} f\right)_{a}(y) \\
& \lesssim 2^{j[s(x)-s(y)]} 2^{j s(y)}\left(\varphi_{j}^{*} f\right)_{a}(y) \\
& \lesssim 2^{j \frac{C_{\log (s)}}{\log (e+1 /|x-y|)}} 2^{j s(y)}\left(\varphi_{j}^{*} f\right)_{a}(y) \lesssim 2^{j s(y)}\left(\varphi_{j}^{*} f\right)_{a}(y),
\end{aligned}
$$

which implies that, for all $x \in P$,

$$
2^{j s(x)}\left|\varphi_{j} * f(x)\right| \lesssim \inf _{y \in P_{j}} 2^{j s(y)}\left(\varphi_{j}^{*} f\right)_{a}(y) .
$$

Thus, choosing $r \in\left(0, \min \left\{1, p_{-}, q_{-}\right\}\right)$and $a$ as in Theorem 3.11, by Theorem 3.11 and Remark 1.1(i), we conclude that

$$
\begin{aligned}
& \mathrm{I}_{P, 1} \lesssim \frac{1}{\phi(P)}\left\|\left\{\sum_{j=0}^{j_{P}-1}\left[\inf _{y \in P_{j}} 2^{j s(y)}\left(\varphi_{j}^{*} f\right)_{a}(y)\right]^{q(\cdot)}\right\}^{\frac{1}{q(\cdot)}}\right\|_{L^{p(\cdot)}(P)} \\
& \lesssim \frac{1}{\phi(P)}\left\|\sum_{j=0}^{j_{P}-1}\right\| 2^{j s(\cdot)}\left(\varphi_{j}^{*} f\right)_{a}\left\|_{L^{p(\cdot)}\left(P_{j}\right)}^{r}\right\| \chi_{P_{j}}\left\|_{L^{p(\cdot)}\left(\mathbb{R}^{n}\right)}^{-r}\right\|_{L^{\frac{p(\cdot)}{r}(P)}}^{\frac{1}{r}} \\
& \lesssim\|f\|_{F_{p(\cdot), q(\cdot)}^{s(\cdot)}}\left\{\mathbb{R}^{\left.j^{n}\right)}\left\{\sum_{j=0}^{j_{P}-1}\left[\frac{\phi\left(P_{j}\right)}{\phi(P)}\right]^{r}\left[\frac{\left\|\chi_{P}\right\|_{L^{p(\cdot)}\left(\mathbb{R}^{n}\right)}}{\left\|\chi_{P_{j}}\right\|_{L^{p(\cdot)}\left(\mathbb{R}^{n}\right)}}\right]^{r}\right\}^{\frac{1}{r}} .\right.
\end{aligned}
$$

On the other hand, by [82, Lemma 2.6], we find that

$$
\left\|\chi_{P_{j}}\right\|_{L^{p(\cdot)}\left(\mathbb{R}^{n}\right)} \gtrsim 2^{-j \frac{n}{p_{+}}} 2^{j_{P} \frac{n}{p_{+}}}\left\|\chi_{P}\right\|_{L^{p(\cdot)}\left(\mathbb{R}^{n}\right)}
$$

and, by the condition $(\mathbf{S} 1)$ of $\phi$, we see that $\phi(P) \geq 2^{j \log _{2} \widetilde{c}_{1}} 2^{-j_{P} \log _{2} \widetilde{c}_{1}} \phi\left(c_{P}, 2^{-j}\right)$, which, together with (3.18) and the condition (S2) of $\phi$, implies that

$$
\begin{aligned}
\mathrm{I}_{P, 1} & \lesssim\|f\|_{F_{p(\cdot), q(\cdot)}^{s(\cdot),}\left(\mathbb{R}^{n}\right)}\left\{\sum_{j=0}^{j} 2^{j\left(\frac{n}{p_{+}}-\log _{2} \widetilde{c}_{1}\right) r}\left[\frac{\phi\left(c_{P}, 2^{-j}\right)}{\phi\left(P_{j}\right)}\right]^{r}\right\}^{\frac{1}{r}} 2^{j_{P}\left(\log _{2} \widetilde{c}_{1}-\frac{n}{p_{+}}\right)} \\
& \lesssim\|f\|_{F_{p(\cdot), q(\cdot)}^{s(\cdot),}\left(\mathbb{R}^{n}\right)}\left\{\sum_{j=0}^{j_{P}-1} 2^{j\left(\frac{n}{p_{+}}-\log _{2} \widetilde{c}_{1}\right) r}\right\}^{\frac{1}{r}} 2^{j_{P}\left(\log _{2} \widetilde{c}_{1}-\frac{n}{p_{+}}\right)} \sim\|f\|_{F_{p(\cdot), q(\cdot)}^{s(\cdot)}\left(\mathbb{R}^{n}\right)},
\end{aligned}
$$


where we used the fact that $\widetilde{c}_{1} \in\left(0,2^{n / p_{+}}\right)$in the last inequality. Therefore,

$$
\left\|f \mid F_{p(\cdot), q(\cdot)}^{s(\cdot),}\left(\mathbb{R}^{n}\right)\right\|_{1}=\sup _{P \in \mathcal{Q}} \mathrm{I}_{P} \lesssim\|f\|_{F_{p(\cdot), q(\cdot)}^{s(\cdot),}\left(\mathbb{R}^{n}\right)},
$$

which completes the proof of (i).

Now we prove (ii). For all $Q \in \mathcal{Q}^{*}$, from (3.17) and Theorem 3.11, we deduce that, for all $x \in Q$,

$$
\begin{aligned}
& {[\phi(Q)]^{-1}\left\|\chi_{Q}\right\|_{L^{p(\cdot)}\left(\mathbb{R}^{n}\right)}|Q|^{-\frac{s(x)}{n}}\left|\varphi_{j_{Q}} * f(x)\right|} \\
& \lesssim \frac{\left\|\chi_{Q}\right\|_{L^{p(\cdot)}\left(\mathbb{R}^{n}\right)}}{\phi(Q)} \inf _{y \in Q}|Q|^{-\frac{s(y)}{n}}\left(\varphi_{j_{Q}}^{*} f\right)_{a}(y) \\
& \lesssim \frac{1}{\phi(Q)}\left\||Q|^{-\frac{s(\cdot)}{n}}\left(\varphi_{j_{Q}}^{*} f\right)_{a}\right\|_{L^{p(\cdot)}(Q)} \lesssim\|f\|_{F_{p(\cdot), q(\cdot)}^{s(\cdot)}\left(\mathbb{R}^{n}\right)}
\end{aligned}
$$

which implies that $\left\|f \mid F_{p(\cdot), q(\cdot)}^{s(\cdot), \phi}\left(\mathbb{R}^{n}\right)\right\|_{2} \lesssim\|f\|_{F_{p(\cdot), q(\cdot)}^{s(\cdot),}\left(\mathbb{R}^{n}\right)}$.

Conversely, by choosing $r \in\left(0, \min \left\{1, p_{-}, q_{-}\right\}\right)$and an argument similar to that used in the proof of (i), we conclude that, for any $P \in \mathcal{Q}$,

$$
\begin{aligned}
& \frac{1}{\phi(P)}\left\|\left\{\sum_{j=\left(j_{P} \vee 0\right)}^{\infty}\left[2^{j s(\cdot)}\left|\varphi_{j} * f\right|\right]^{q(\cdot)}\right\}^{\frac{1}{q(\cdot)}}\right\|_{L^{p(\cdot)}(P)} \\
& \lesssim\left\|f \mid F_{p(\cdot), q(\cdot)}^{s(\cdot),}\left(\mathbb{R}^{n}\right)\right\|_{2} \|\left\{\sum_{j=\left(j_{P} \vee 0\right)}^{\infty}\left[\sum_{\substack{\ell(\widetilde{Q})=2-j \\
\widetilde{Q} \in \mathcal{Q}, \widetilde{Q} \subset P}} \frac{\phi(P)^{-1} \phi(\widetilde{Q}) \chi_{\widetilde{Q}}}{\left\|\chi_{\widetilde{Q}}\right\|_{L^{p(\cdot)}\left(\mathbb{R}^{n}\right)}}\right]^{q(\cdot)}\left\|_{L^{p(\cdot)(P)}}^{\frac{1}{q(\cdot)}}\right\|\right. \\
& \lesssim\left\|f \mid F_{p(\cdot), q(\cdot)}^{s(\cdot), \phi}\left(\mathbb{R}^{n}\right)\right\|_{2}\left\{\sum_{j=\left(j_{P} \vee 0\right)}^{\infty} 2^{j\left(\frac{n}{p_{-}}+\log _{2} c_{1}\right) r}\right\}^{\frac{1}{r}} 2^{-j_{P}\left(\frac{n}{p_{-}}+\log _{2} c_{1}\right)} \\
& \lesssim\left\|f \mid F_{p(\cdot), q(\cdot)}^{s(\cdot),}\left(\mathbb{R}^{n}\right)\right\|_{2},
\end{aligned}
$$

where we used the fact that $c_{1} \in\left(0,2^{-n / p_{-}}\right)$in the last inequality, which implies that

$$
\|f\|_{F_{p(\cdot), q(\cdot)}^{s(\cdot),}\left(\mathbb{R}^{n}\right)} \lesssim\left\|f \mid F_{p(\cdot), q(\cdot)}^{s(\cdot), \phi}\left(\mathbb{R}^{n}\right)\right\|_{2}
$$

This finishes the proof of (ii) and hence Theorem 3.12.

Remark 3.13. In the case that $p, q, s$ and $\phi$ are as in Remark 1.5(ii), Theorem 3.12(i) coincides with [80, Corollary 3.3(i)] and Theorem 3.12(ii) goes back to [78, Theorem 2.2(i)].

We now compare the Triebel-Lizorkin-type space with variable exponents in this article with the variable Triebel-Lizorkin-Morrey space $\mathcal{E}_{p(\cdot), q(\cdot), u}^{s(\cdot)}\left(\mathbb{R}^{n}\right)$ introduced by Ho [31] and show that, in general, these two scales of Triebel-Lizorkin spaces do not cover each other. 
To recall the definition of the variable Triebel-Lizorkin-Morrey space in [31], we need some notions. A measurable function $u(x, r): \mathbb{R}^{n} \times(0, \infty) \rightarrow(0, \infty)$ is said to belong to $\mathcal{W}_{q}$ with $q \in(0, \infty)$ if there exist $C_{1}, C_{2} \in(0, \infty)$ and $\lambda \in[0,1 / q)$ such that, for all $x \in \mathbb{R}^{n}, u(x, r)>1$ if $r \in[1, \infty), \frac{u(x, 2 r)}{u(x, r)} \leq 4^{n \lambda}$ if $r \in(0, \infty)$, and

$$
C_{2}^{-1} \leq \frac{u(x, t)}{u(x, r)} \leq C_{2} \quad \text { if } \quad 0<r \leq t \leq 2 r .
$$

Definition 3.14. Let $p, q, s,\left\{\varphi_{j}\right\}_{j \in \mathbb{Z}_{+}}$be as in Definition 1.4 and $u \in \mathcal{W}_{p_{+}}$. Then the variable Triebel-Lizorkin-Morrey space $\mathcal{E}_{p(\cdot), q(\cdot), u}^{s(\cdot)}\left(\mathbb{R}^{n}\right)$ is defined to be the set of all $f \in \mathcal{S}^{\prime}\left(\mathbb{R}^{n}\right)$ such that

$$
\|f\|_{\mathcal{E}_{p(\cdot), q(\cdot), u}^{s(\cdot)}\left(\mathbb{R}^{n}\right)}:=\sup _{\substack{z \in \mathbb{R}^{n} \\ R \in(0, \infty)}} \frac{1}{u(z, R)}\left\|\left\{\sum_{j=0}^{\infty}\left[2^{j s(\cdot)}\left|\varphi_{j} * f\right|\right]^{q(\cdot)}\right\}^{\frac{1}{q(\cdot)}}\right\|_{L^{p(\cdot)}(B(z, R))}<\infty .
$$

Remark 3.15. (i) We point out that the Triebel-Lizorkin-type space with variable exponents in this article can not be covered by the Triebel-Lizorkin-Morrey space in [31] even when $\widetilde{c}_{1} \in\left(0,2^{n / p_{+}}\right)$. To see this, it suffices to show that there exists a set function $\phi$ satisfying $(\mathbf{S 1})$ and $(\mathbf{S 2})$ does not belong to $\mathcal{W}_{q}$ for any $q \in(0, \infty)$.

Indeed, for all $Q \subset \mathbb{R}^{n}$, let $\phi(Q):=\int_{Q}|x|^{\alpha} d x$, where $\alpha \in(-n, 0)$. Then, by [65, p. 196], we know that $\phi$ is doubling, which, together with Remark 1.3(iv), further implies that $\phi$ satisfies the conditions $(\mathbf{S} 1)$ and $(\mathbf{S} 2)$. However, $\phi \notin \mathcal{W}_{q}$ for any $q \in(0, \infty)$. To see this, let $x_{0} \in \mathbb{R}^{n}$ and $r \in(1,2)$ satisfy $\left|x_{0}\right| \geq 2 r$. Then

$$
\phi\left(x_{0}, r\right):=\phi\left(Q\left(x_{0}, r\right)\right)=\int_{Q\left(x_{0}, r\right)}|y|^{\alpha} d y \sim\left|x_{0}\right|^{\alpha}
$$

tends 0 as $\left|x_{0}\right| \rightarrow \infty$ since $\alpha \in(-n, 0)$, which implies that $\phi \notin \mathcal{W}_{q}$ for any $q \in(0, \infty)$.

(ii) Also, the variable Triebel-Lizorkin-Morrey space investigated in [31] can not be covered by the Triebel-Lizorkin-type space with variable exponents in this article. To see this, it suffices to show that there exists a function $u$ such that $u$ belongs to $\mathcal{W}_{1}$ but does not satisfy the condition $(\mathbf{S 2})$.

Indeed, let, for all $x \in \mathbb{R}^{n}$ and $r \in(0, \infty), u(x, r):=r^{\lambda(x)}$, where $\lambda(x):=$ $n\left(1-\frac{1}{1+|x|}\right)$. Then, as was pointed out in [31, p.380], $u \in \mathcal{W}_{1}$. However, $u$ does not satisfy the condition (S2). To see this, let $x, y \in \mathbb{R}^{n}$ satisfy that $\varepsilon<|x|<\frac{(1+\varepsilon) r+\varepsilon}{2+\varepsilon}$ and $|y|=\frac{1+|x|}{1+\varepsilon}-1$, where $\varepsilon \in(0, \infty)$ and $r \in(\varepsilon, \infty)$. Then

$$
|x-y| \leq|x|+|y|=|x|+\frac{1+|x|}{1+\varepsilon}-1<r
$$

but

$$
\frac{u(x, r)}{u(y, r)}=r^{n\left(\frac{1}{1+|y|}-\frac{1}{1+|x|}\right)}=r^{\frac{n \varepsilon}{1+|x|}} \rightarrow \infty, \quad \text { as } \quad r \rightarrow \infty,
$$

which implies that $u$ does not satisfy the condition $(\mathbf{S 2})$.

As an application of Theorem 3.12, we prove that the space $F_{p(\cdot), 2}^{0, \phi}\left(\mathbb{R}^{n}\right)$ coincides with the Morrey space with variable exponent, $\mathcal{M}_{\phi}^{p(\cdot)}\left(\mathbb{R}^{n}\right)$, which is defined to be 
the set of all measurable functions $f$ such that

$$
\|f\|_{\mathcal{M}_{\phi}^{p(\cdot)}\left(\mathbb{R}^{n}\right)}:=\sup _{P \in \mathcal{Q}} \frac{1}{\phi(P)}\|f\|_{L^{p(\cdot)}(P)}<\infty
$$

where the supremum is taken over all dyadic cubes of $\mathbb{R}^{n}$.

Remark 3.16. (i) We point out that, in [31], Ho studied the variable Morrey space $\mathcal{M}_{u}^{p(\cdot)}\left(\mathbb{R}^{n}\right)$, which is defined in the same way as $\mathcal{M}_{\phi}^{p(\cdot)}\left(\mathbb{R}^{n}\right)$ above but with $\phi$ replaced by $u$ as in Definition 3.14 and the supremum is taken over all balls of $\mathbb{R}^{n}$. From Remark 3.15, we deduce that the Morrey space with variable exponent $\mathcal{M}_{\phi}^{p(\cdot)}\left(\mathbb{R}^{n}\right)$ in this article and the variable Morrey space $\mathcal{M}_{u}^{p(\cdot)}\left(\mathbb{R}^{n}\right)$ in [31] do not cover each other.

(ii) For $\varphi: \mathbb{R}^{n} \times(0, \infty) \rightarrow(0, \infty)$ and a variable exponent $p: \mathbb{R}^{n} \rightarrow[1, \infty)$, Nakai [52] introduced the variable Morrey space $L^{(p, \varphi)}\left(\mathbb{R}^{n}\right)$, which is defined to be the set of all measurable functions $f$ such that

$$
\|f\|_{L^{(p, \varphi)}\left(\mathbb{R}^{n}\right)}:=\sup _{\text {balls } B \subset \mathbb{R}^{n}}\|f\|_{p, \varphi, B}<\infty,
$$

where, for all balls $B:=B(x, r) \subset \mathbb{R}^{n}, \varphi(B):=\varphi(x, r)$ and

$$
\|f\|_{p, \varphi, B}:=\inf \left\{\lambda \in(0, \infty): \frac{1}{\varphi(B)|B|} \int_{B}\left[\frac{|f(y)|}{\lambda}\right]^{p(y)} d y \leq 1\right\},
$$

and the supremum is taken over all balls $B$ of $\mathbb{R}^{n}$.

We claim that, if there exists a positive constant $C$ such that, for all $x \in \mathbb{R}^{n}$ and $0<r<s<\infty$,

$$
C^{-1} \phi(x, r) \leq \phi(x, s) \leq C \phi(x, r)
$$

and, for all balls $B \subset \mathbb{R}^{n}$ and all $y \in B$,

$$
\varphi(B)|B| \sim[\phi(B)]^{p(y)},
$$

then $\mathcal{M}_{\phi}^{p(\cdot)}\left(\mathbb{R}^{n}\right)$ coincides with $L^{(p, \varphi)}\left(\mathbb{R}^{n}\right)$.

Indeed, by (3.19) and the definition of $\|\cdot\|_{\mathcal{M}_{\phi}^{p(\cdot)}\left(\mathbb{R}^{n}\right)}$, we conclude that

$$
\|f\|_{\mathcal{M}_{\phi}^{p(\cdot)}\left(\mathbb{R}^{n}\right)} \sim \sup _{\text {balls } B \subset \mathbb{R}^{n}} \inf \left\{\lambda \in(0, \infty): \int_{B}\left[\frac{|f(y)|}{\phi(B) \lambda}\right]^{p(y)} d y \leq 1\right\} .
$$

On the other hand, by (3.20), we find that

$$
\begin{aligned}
& \inf \left\{\lambda \in(0, \infty): \int_{B}\left[\frac{|f(y)|}{\phi(B) \lambda}\right]^{p(y)} d y \leq 1\right\} \\
& \sim \inf \left\{\lambda \in(0, \infty): \frac{1}{\varphi(B)|B|} \int_{B}\left[\frac{|f(y)|}{\lambda}\right]^{p(y)} d y \leq 1\right\}
\end{aligned}
$$

which, combined with $(3.21)$, implies that $\mathcal{M}_{\phi}^{p(\cdot)}\left(\mathbb{R}^{n}\right)$ coincides with $L^{(p, \varphi)}\left(\mathbb{R}^{n}\right)$. This proves the above claim.

Obviously, in general, these two scales of Morrey spaces with variable exponents, $\mathcal{M}_{\phi}^{p(\cdot)}\left(\mathbb{R}^{n}\right)$ and $L^{(p, \varphi)}\left(\mathbb{R}^{n}\right)$, may not cover each other. 
In what follows, for all $p \in \mathcal{P}\left(\mathbb{R}^{n}\right)$, denote by $L^{p(\cdot)}\left(\ell^{2}\left(\mathbb{R}^{n}\right)\right)$ the set of all sequences $\left\{g_{j}\right\}_{j \in \mathbb{Z}_{+}}$of measurable functions such that

$$
\left\|\left\{g_{j}\right\}_{j \in \mathbb{Z}_{+}}\right\|_{L^{p(\cdot)\left(\ell^{2}\left(\mathbb{R}^{n}\right)\right)}}:=\|\left.\left\{\sum_{j \in \mathbb{Z}_{+}}\left|g_{j}\right|^{2}\right\}^{\frac{1}{2}}\right|_{L^{p(\cdot)\left(\mathbb{R}^{n}\right)}}<\infty .
$$

Let $(\varphi, \Phi)$ and $(\psi, \Psi)$ be two pairs of admissible functions satisfying (2.1). The operator $\mathcal{G}$ is defined by setting, for all $f \in L^{p(\cdot)}\left(\mathbb{R}^{n}\right), \mathcal{G}(f):=\left\{\varphi_{j} * f\right\}_{j \in \mathbb{Z}_{+}}$, where, when $j=0, \varphi_{0}$ is replaced by $\Phi$, and its conjugate operator $\mathcal{G}^{*}$ is defined by setting, for all $\left\{g_{j}\right\}_{j \in \mathbb{Z}_{+}} \in L^{\widetilde{P}(\cdot)}\left(\ell^{2}\left(\mathbb{R}^{n}\right)\right)$,

$$
\mathcal{G}^{*}\left(\left\{g_{j}\right\}_{j \in \mathbb{Z}_{+}}\right):=\sum_{j \in \mathbb{Z}_{+}} \psi_{j} * g_{j},
$$

where, when $j=0, \psi_{0}$ is replaced by $\Psi$.

Remark 3.17. Let $p(\cdot) \in C^{\log }\left(\mathbb{R}^{n}\right)$ satisfy $1<p_{-} \leq p_{+}<\infty$. Then, from the fact that $L^{\widetilde{P}(\cdot)}\left(\mathbb{R}^{n}\right)=F_{\widetilde{P}(\cdot), 2}^{0}\left(\mathbb{R}^{n}\right)$ (see $[20$, Theorem 4.2$\left.]\right)$, we deduce that the operator $\mathcal{G}$ is bounded from $L^{\widetilde{P}(\cdot)}\left(\mathbb{R}^{n}\right)$ to $L^{\widetilde{P}(\cdot)}\left(\ell^{2}\left(\mathbb{R}^{n}\right)\right)$. Furthermore, by an argument similar to that used in the proof of [31, Corollary 4.4], we conclude that the operator $\mathcal{G}^{*}$ is bounded from $L^{p(\cdot)}\left(\ell^{2}\left(\mathbb{R}^{n}\right)\right)$ to $L^{p(\cdot)}\left(\mathbb{R}^{n}\right)$.

Proposition 3.18. Let $p$ and $\phi$ be as in Definition 1.4 and $\widetilde{c}_{1} \in\left(0,2^{n / p_{+}}\right)$. If $1<p_{-} \leq p_{+}<\infty$, then

$$
\mathcal{M}_{\phi}^{p(\cdot)}\left(\mathbb{R}^{n}\right)=F_{p(\cdot), 2}^{0, \phi}\left(\mathbb{R}^{n}\right)
$$

with equivalent norms.

Proof. We first prove that $\mathcal{M}_{\phi}^{p(\cdot)}\left(\mathbb{R}^{n}\right) \hookrightarrow F_{p(\cdot), 2}^{0, \phi}\left(\mathbb{R}^{n}\right)$. By Theorem 3.12(i), it suffices to show that, for all $f \in \mathcal{M}_{\phi}^{p(\cdot)}\left(\mathbb{R}^{n}\right)$,

$$
\sup _{Q \in \mathcal{Q}} \frac{1}{\phi(Q)}\left\|\left\{\sum_{j=0}^{\infty}\left|\varphi_{j} * f\right|^{2}\right\}^{\frac{1}{2}}\right\|_{L^{p(\cdot)}(Q)} \lesssim\|f\|_{\mathcal{M}_{\phi}^{p(\cdot)}\left(\mathbb{R}^{n}\right)}
$$

where $\left\{\varphi_{j}\right\}_{j=0}^{\infty}$ are as in Definition 1.4.

For all $Q:=Q\left(x_{0}, r\right) \in \mathcal{Q}$, let $f_{1}:=f \chi_{Q\left(x_{0}, 2 r\right)}$ and $f_{2}:=f-f_{1}$. From [20, Theorem 4.2] and the condition $(\mathbf{S 1})$ of $\phi$, we deduce that

$$
\begin{aligned}
\mathrm{I}_{1} & :=\frac{1}{\phi(Q)}\left\|\left\{\sum_{j=0}^{\infty}\left|\varphi_{j} * f_{1}\right|^{2}\right\}^{\frac{1}{2}}\right\|_{L^{p(\cdot)}(Q)} \\
& \lesssim \frac{1}{\phi(Q)}\left\|f_{1}\right\|_{L^{p(\cdot)}\left(\mathbb{R}^{n}\right)} \sim \frac{1}{\phi(Q)}\|f\|_{L^{p(\cdot)}\left(Q\left(x_{0}, 2 r\right)\right)} \lesssim\|f\|_{\mathcal{M}_{\phi}^{p(\cdot)}\left(\mathbb{R}^{n}\right)}
\end{aligned}
$$

On the other hand, by the Minkowski inequality, we find that, for all $x \in \mathbb{R}^{n}$,

$$
\left\{\sum_{j=0}^{\infty}\left|\varphi_{j} * f_{2}(x)\right|^{2}\right\}^{\frac{1}{2}} \lesssim \int_{\mathbb{R}^{n} \backslash Q\left(x_{0}, 2 r\right)}\left\{\sum_{j=0}^{\infty}\left|\varphi_{j}(x-y)\right|^{2}\right\}^{\frac{1}{2}}|f(y)| d y
$$




$$
\lesssim \int_{\mathbb{R}^{n} \backslash Q\left(x_{0}, 2 r\right)} \frac{|f(y)|}{|x-y|^{n}} d y \sim \sum_{k=1}^{\infty} \int_{S_{k}} \frac{|f(y)|}{|x-y|^{n}} d y
$$

where, for $k \in \mathbb{N}, S_{k}:=Q_{k+1} \backslash Q_{k}$ and $Q_{k}:=Q\left(x_{0}, 2^{k} r\right)$. Observe that, when $x \in Q\left(x_{0}, r\right)$ and $y \in S_{k},|x-y| \geq 2^{k} r$. Setting $(p(\cdot))^{*}:=\frac{p(\cdot)}{p(\cdot)-1}$, by the Hölder inequality of variable Lebesgue spaces (see Remark 1.1(iii)), [82, Lemma 2.6] and [31, Proposition 2.4], we see that

$$
\begin{aligned}
& \left\|\left\{\sum_{j=0}^{\infty}\left|\varphi_{j} * f_{2}\right|^{2}\right\}^{\frac{1}{2}}\right\|_{L^{p(\cdot)}(Q)} \\
& \quad \lesssim \sum_{k=1}^{\infty} \frac{1}{2^{k n} r^{n}}\|f\|_{L^{p(\cdot)}\left(S_{k}\right)}\left\|\chi_{S_{k}}\right\|_{L^{p(\cdot)^{*}\left(\mathbb{R}^{n}\right)}}\left\|\chi_{Q}\right\|_{L^{p(\cdot)}\left(\mathbb{R}^{n}\right)} \\
& \quad \lesssim \sum_{k=1}^{\infty} \frac{2^{-k n / p_{+}}}{r^{n} 2^{k n}}\|f\|_{L^{p(\cdot)}\left(S_{k}\right)}\left\|\chi_{Q_{k+1}}\right\|_{L^{p(\cdot)^{*}}\left(\mathbb{R}^{n}\right)}\left\|\chi_{Q_{k+1}}\right\|_{L^{p(\cdot)}\left(\mathbb{R}^{n}\right)} \\
& \quad \lesssim \sum_{k=1}^{\infty} 2^{-k n / p_{+}}\|f\|_{L^{p(\cdot)}\left(S_{k}\right)},
\end{aligned}
$$

which, together with the condition $(\mathbf{S 1})$ of $\phi$, implies that

$$
\begin{aligned}
\mathrm{I}_{2} & :=\frac{1}{\phi(Q)}\left\|\left\{\sum_{j=0}^{\infty}\left|\varphi_{j} * f_{2}\right|^{2}\right\}^{\frac{1}{2}}\right\|_{L^{p(\cdot)}(Q)} \lesssim \sum_{k=1}^{\infty} 2^{-k n / p_{+}} \frac{\|f\|_{L^{p(\cdot)}\left(Q_{k+1}\right)} \frac{\phi\left(Q_{k+1}\right)}{\phi\left(Q_{k+1}\right)}}{\phi(Q)} \\
& \lesssim\|f\|_{\mathcal{M}_{\phi}^{p(\cdot)}\left(\mathbb{R}^{n}\right)} \sum_{k=1}^{\infty} 2^{-k n / p_{+}} 2^{k \log _{2} \widetilde{c}_{1}} \sim\|f\|_{\mathcal{M}_{\phi}^{p(\cdot)}\left(\mathbb{R}^{n}\right)}
\end{aligned}
$$

where we used the fact that $\widetilde{c}_{1} \in\left(0,2^{n / p_{+}}\right)$in the last inequality.

Combining (3.23) and (3.24), we conclude that

$$
\sup _{Q \in \mathcal{Q}} \frac{1}{\phi(Q)}\left\|\left\{\sum_{j=0}^{\infty}\left|\varphi_{j} * f\right|^{2}\right\}^{\frac{1}{2}}\right\|\left\|_{L^{p(\cdot)}(Q)} \lesssim \sup _{Q \in \mathcal{Q}}\left(\mathrm{I}_{1}+\mathrm{I}_{2}\right) \lesssim\right\| f \|_{\mathcal{M}_{\phi}^{p(\cdot)}\left(\mathbb{R}^{n}\right)}
$$

and (3.22) holds true.

Next, we prove that $F_{p(\cdot), 2}^{0, \phi}\left(\mathbb{R}^{n}\right) \hookrightarrow \mathcal{M}_{\phi}^{p(\cdot)}\left(\mathbb{R}^{n}\right)$. Let $f \in F_{p(\cdot), 2}^{0, \phi}\left(\mathbb{R}^{n}\right)$. Then, by the Calderón reproducing formula (see [80, Lemma 2.3]), we find that

$$
f=\Psi * \Phi * f+\sum_{j=1}^{\infty} \psi_{j} * \varphi_{j} * f=: \sum_{j=0}^{\infty} \psi_{j} * \varphi_{j} * f
$$

in $\mathcal{S}^{\prime}\left(\mathbb{R}^{n}\right)$, where $\Psi, \Phi, \varphi$ and $\psi$ are as in (2.1). For all $j \in \mathbb{Z}_{+}$, we use $f_{j}$ to denote $\varphi_{j} * f$. For all $Q:=Q\left(x_{0}, r\right) \in \mathcal{Q}$ and $j \in \mathbb{Z}_{+}$, let $f_{j}^{1}:=f_{j} \chi_{Q\left(x_{0}, 2 r\right)}$ and 
$f_{j}^{2}:=f_{j}-f_{j}^{1}$. Then we know that

$$
\begin{aligned}
& \frac{1}{\phi(Q)}\left\|\sum_{j=0}^{\infty} \psi_{j} * \varphi_{j} * f\right\|_{L^{p(\cdot)}(Q)} \\
& \lesssim \frac{1}{\phi(Q)}\left\|\sum_{j=0}^{\infty} \psi_{j} * f_{j}^{1}\right\|_{L^{p(\cdot)}(Q)}+\frac{1}{\phi(Q)}\left\|\sum_{j=0}^{\infty} \psi_{j} * f_{j}^{2}\right\|_{L^{p(\cdot)}(Q)}=: \mathrm{J}_{1}+\mathrm{J}_{2} .
\end{aligned}
$$

By Remark 3.17, the condition (S1) of $\phi$ and Theorem 3.12(i), we see that

$$
\begin{aligned}
\mathrm{J}_{1} & \sim \frac{1}{\phi(Q)}\left\|\mathcal{G}^{*}\left(\left\{f_{j}^{1}\right\}_{j \in \mathbb{Z}_{+}}\right)\right\|_{L^{p(\cdot)}\left(\mathbb{R}^{n}\right)} \\
& \lesssim \frac{1}{\phi(Q)}\left\|\left\{\sum_{j=0}^{\infty}\left|\varphi_{j} * f\right|^{2}\right\}^{\frac{1}{2}}\right\|_{L^{p(\cdot)}\left(Q\left(x_{0}, 2 r\right)\right)} \lesssim\|f\|_{F_{p(\cdot), 2}^{0, \phi}\left(\mathbb{R}^{n}\right)} .
\end{aligned}
$$

On the other hand, for all $x \in Q\left(x_{0}, 2 r\right)$, by the Hölder inequality, we find that

$$
\left|\sum_{j \in \mathbb{Z}_{+}} \psi_{j} * f_{j}^{2}(x)\right| \lesssim \int_{\mathbb{R}^{n} \backslash Q\left(x_{0}, 2 r\right)}\left[\sum_{j \in \mathbb{Z}_{+}}\left|f_{j}(y)\right|^{2}\right]^{\frac{1}{2}}|x-y|^{-n} d y .
$$

Thus, by an argument similar to that used in the proof of (3.24), we conclude that

$$
\mathrm{J}_{2} \lesssim\|f\|_{F_{p(\cdot), 2}^{0, \phi}\left(\mathbb{R}^{n}\right)}
$$

which, combined with (3.26), implies that

$$
\frac{1}{\phi(Q)}\left\|\sum_{j=0}^{\infty} \psi_{j} * \varphi_{j} * f\right\|_{L^{p(\cdot)}(Q)} \lesssim \mathrm{J}_{1}+\mathrm{J}_{2} \lesssim\|f\|_{F_{p(\cdot), 2}^{0, \phi}\left(\mathbb{R}^{n}\right)} .
$$

Therefore, $\sum_{j=0}^{\infty} \psi_{j} * \varphi_{j} * f \in \mathcal{M}_{\phi}^{p(\cdot)}\left(\mathbb{R}^{n}\right)$ and

$$
\left\|\sum_{j=0}^{\infty} \psi_{j} * \varphi_{j} * f\right\|_{\mathcal{M}_{\phi}^{p(\cdot)}\left(\mathbb{R}^{n}\right)} \lesssim\|f\|_{F_{p(\cdot), 2}^{0, \phi}\left(\mathbb{R}^{n}\right)},
$$

which, together with (3.25), implies that $F_{p(\cdot), 2}^{0, \phi}\left(\mathbb{R}^{n}\right) \hookrightarrow \mathcal{M}_{\phi}^{p(\cdot)}\left(\mathbb{R}^{n}\right)$. This finishes the proof of Proposition 3.18.

Remark 3.19. In the case that $p$ and $\phi$ are as in Remark 1.5(ii), the conclusion of Proposition 3.18 is already known; see, for example, [62, Theorem 3.9].

We end this section by giving another application of Theorem 3.11.

Proposition 3.20. Let $p, q, s$ and $\phi$ be as in Definition 1.4. Then

$$
\mathcal{S}\left(\mathbb{R}^{n}\right) \hookrightarrow F_{p(\cdot), q(\cdot)}^{s(\cdot),}\left(\mathbb{R}^{n}\right) \hookrightarrow \mathcal{S}^{\prime}\left(\mathbb{R}^{n}\right) .
$$


Proof. We first prove that $\mathcal{S}\left(\mathbb{R}^{n}\right) \hookrightarrow F_{p(\cdot), q(\cdot)}^{s(\cdot),}\left(\mathbb{R}^{n}\right)$. To prove this embedding, we need to show that there exists an $M \in \mathbb{N}$ such that, for all $f \in \mathcal{S}\left(\mathbb{R}^{n}\right)$,

$$
\|f\|_{F_{p(\cdot), q(\cdot)}^{s(\cdot),}\left(\mathbb{R}^{n}\right)} \lesssim\|f\|_{\mathcal{S}_{M}\left(\mathbb{R}^{n}\right)}
$$

Let $f \in \mathcal{S}\left(\mathbb{R}^{n}\right)$ and $(\varphi, \Phi)$ be a pair of admissible functions. Let $P:=Q_{j_{P} k_{P}}$ be an arbitrary dyadic cube. If $j_{P}>0$, choosing $r \in\left(0, \min \left\{1, p_{-}, q_{-}\right\}\right)$, by [80, Lemma 2.4], Remark 1.1(i) and Lemmas 2.6 and 2.7, we obtain

$$
\begin{aligned}
& \frac{1}{\phi(P)}\left\|\left\{\sum_{j=j_{P}}^{\infty}\left[2^{j s(\cdot)}\left|\varphi_{j} * f\right|\right]^{q(\cdot)}\right\}^{\frac{1}{q(\cdot)}}\right\|_{L^{p(\cdot)}(P)} \\
& \lesssim\|f\|_{\mathcal{S}_{M+1}\left(\mathbb{R}^{n}\right)} \frac{1}{\phi(P)}\left\|\left\{\sum_{j=j_{P}}^{\infty}\left[2^{j s(\cdot)} \frac{2^{-j M}}{(1+|\cdot|)^{n+M}}\right]^{q(\cdot)}\right\}^{\frac{1}{q(\cdot)}}\right\|_{L^{p(\cdot)}(P)} \\
& \lesssim\|f\|_{\mathcal{S}_{M+1}\left(\mathbb{R}^{n}\right)} \frac{1}{\phi(P)}\left\{\sum_{j=j_{P}}^{\infty} 2^{-j\left(M-s_{+}\right) r}\left\|\frac{1}{(1+|\cdot|)^{(M+n) r}}\right\|_{L^{\frac{p(\cdot)}{r}(P)}}\right\}^{\frac{1}{r}} \\
& \lesssim\|f\|_{\mathcal{S}_{M+1}\left(\mathbb{R}^{n}\right)}\left\{\sum_{j=j_{P}}^{\infty} 2^{-j\left(M-s_{+}\right) r} \frac{1}{\left(1+2^{-j_{P}}\left|k_{P}\right|\right)^{(M+n) r}}\right\}^{\frac{1}{r}} \frac{\left\|\chi_{P}\right\|_{L^{p(\cdot)}(P)}}{\phi(P)} \\
& \lesssim\|f\|_{\mathcal{S}_{M+1}\left(\mathbb{R}^{n}\right)} 2^{-j_{P}\left(\frac{M}{2}+\frac{n}{p_{+}}-s_{+}-\log _{2} c_{1}\right)}\left(1+\left|k_{P}\right|\right)^{-\frac{M}{2}+n\left(\frac{1}{p_{-}}-\frac{1}{p_{+}}\right)+\log _{2}\left(c_{1} \widetilde{c}_{1}\right)} \\
& \lesssim\|f\|_{\mathcal{S}_{M+1}\left(\mathbb{R}^{n}\right)} \text {, }
\end{aligned}
$$

where $M$ is chosen large enough.

If $j_{P} \leq 0$, then we see that

$$
\begin{aligned}
\mathrm{I}_{P} & :=\frac{1}{\phi(P)}\left\|\left\{\sum_{j=0}^{\infty}\left[2^{j s(\cdot)}\left|\varphi_{j} * f\right|\right]^{q(\cdot)}\right\}^{\frac{1}{q(\cdot)}}\right\|_{L^{p(\cdot)}(P)} \\
& \lesssim \frac{1}{\phi(P)}\|\Phi * f\|_{L^{p(\cdot)}(P)}+\frac{1}{\phi(P)}\left\{\sum_{j=1}^{\infty} 2^{j s_{+} r}\left\|\varphi_{j} * f\right\|_{L^{p(\cdot)}(P)}^{r}\right\}^{\frac{1}{r}} .
\end{aligned}
$$

When $P$ is away from the origin, by an argument similar to that used in the proof of (3.27), we conclude that $\mathrm{I}_{P} \lesssim\|f\|_{\mathcal{S}_{M+1}\left(\mathbb{R}^{n}\right)}$ with $M$ being sufficiently large. When one of the corners of $P$ is the origin, then $P \subset \cup_{i=0}^{-j_{P}+n} S_{i}$, where $S_{0}:=B(0,1)$ and $S_{i}:=2^{i} S_{0} \backslash\left(2^{i-1} S_{0}\right)$ for all $i \in\left\{1, \ldots,-j_{P}+1\right\}$. From this, Lemmas 2.6 and 2.7 and the fact that $\left|k_{P}\right| \leq 1$, we deduce that

$$
\frac{1}{\phi(P)}\|\Phi * f\|_{L^{p(\cdot)}(P)} \lesssim \frac{1}{\phi(P)}\left\{\sum_{i=0}^{-j_{P}+n}\left\|\frac{1}{(1+|\cdot|)^{M}}\right\|_{L^{p(\cdot)}\left(S_{i}\right)}^{r}\right\}^{\frac{1}{r}} \lesssim\|f\|_{\mathcal{S}_{M+1}\left(\mathbb{R}^{n}\right)}
$$


and, similarly,

$$
\frac{1}{\phi(P)}\left\{\sum_{j=1}^{\infty} 2^{j s_{+} r}\left\|\varphi_{j} * f\right\|_{L^{p(\cdot)}(P)}^{r}\right\}^{\frac{1}{r}} \lesssim\|f\|_{\mathcal{S}_{M+1}\left(\mathbb{R}^{n}\right)},
$$

where $M$ is chosen large enough, which implies that $\mathrm{I}_{P} \lesssim\|f\|_{\mathcal{S}_{M+1}\left(\mathbb{R}^{n}\right)}$. Therefore, $\mathcal{S}\left(\mathbb{R}^{n}\right) \hookrightarrow F_{p(\cdot), q(\cdot)}^{s(\cdot), \phi}\left(\mathbb{R}^{n}\right)$ and $\|f\|_{F_{p(\cdot), q(\cdot)}^{s(\cdot)}} \lesssim\|f\|_{\mathcal{S}_{M+1}\left(\mathbb{R}^{n}\right)}$.

Next we show that $F_{p(\cdot), q(\cdot)}^{s(\cdot)}\left(\mathbb{R}^{n}\right) \hookrightarrow \mathcal{S}^{\prime}\left(\mathbb{R}^{n}\right)$. To this end, we need to prove that there exists an $M \in \mathbb{N}$ such that, for all $f \in F_{p(\cdot), q(\cdot)}^{s(\cdot),}\left(\mathbb{R}^{n}\right)$ and $h \in \mathcal{S}\left(\mathbb{R}^{n}\right)$,

$$
|\langle f, h\rangle| \lesssim\|f\|_{F_{p(\cdot), q(\cdot)}^{s(\cdot),}\left(\mathbb{R}^{n}\right)}\|h\|_{\mathcal{S}_{M+1}\left(\mathbb{R}^{n}\right)} \cdot
$$

Let $\varphi, \psi, \Phi$ and $\Psi$ be as in Theorem 2.3. Then, by the Calderón reproducing formula in [80, Lemma 2.3], together with [80, Lemma 2.4], we obtain

$$
\begin{aligned}
|\langle f, h\rangle| & \leq \int_{\mathbb{R}^{n}}|\Phi * f(x)||\Psi * h(x)| d x+\sum_{j=1}^{\infty} \int_{\mathbb{R}^{n}}\left|\varphi_{j} * f(x)\right|\left|\psi_{j} * h(x)\right| d x \\
& \lesssim\|h\|_{\mathcal{S}_{M+1}\left(\mathbb{R}^{n}\right)} \sum_{j=0}^{\infty} 2^{-j M} \int_{\mathbb{R}^{n}}\left|\varphi_{j} * f(x)\right|(1+|x|)^{-(n+M)} d x \\
& \sim\|h\|_{\mathcal{S}_{M+1}\left(\mathbb{R}^{n}\right)} \sum_{j=0}^{\infty} 2^{-j M} \sum_{k \in \mathbb{Z}^{n}} \int_{Q_{0 k}}\left|\varphi_{j} * f(x)\right|(1+|x|)^{-(n+M)} d x
\end{aligned}
$$

where we used $\Phi$ to replace $\varphi_{0}$ and chose $M>a$. Notice that, for any $j \in \mathbb{Z}_{+}$, $k \in \mathbb{Z}^{n}, a \in(0, \infty)$ and $y \in Q_{j k}$,

$$
\begin{aligned}
\int_{Q_{0 k}}\left|\varphi_{j} * f(x)\right| d x & \lesssim\left(\varphi_{j}^{*} f\right)_{a}(y) \int_{Q_{0 k}}\left(1+2^{j}|x|+2^{j}|y|\right)^{a} d x \\
& \lesssim 2^{j a}\left(\varphi_{j}^{*} f\right)_{a}(y)(1+|k|)^{a} .
\end{aligned}
$$

Then, by the arbitrariness of $y \in Q_{j k}$, we see that

$$
\int_{Q_{0 k}}\left|\varphi_{j} * f(x)\right| d x \lesssim 2^{j a}(1+|k|)^{a} \inf _{y \in Q_{j k}}\left(\varphi_{j}^{*} f\right)_{a}(y),
$$

which, combined with (3.28), Theorem 3.11 and Lemmas 2.6 and 2.7, implies that

$$
\begin{aligned}
|\langle f, h\rangle| & \lesssim\|h\|_{\mathcal{S}_{M+1}\left(\mathbb{R}^{n}\right)} \sum_{j=0}^{\infty} 2^{-j M} \sum_{k \in \mathbb{Z}^{n}} \int_{Q_{0 k}} \frac{\left|\varphi_{j} * f(x)\right|}{(1+|k|)^{n+M}} d x \\
& \lesssim\|h\|_{\mathcal{S}_{M+1}\left(\mathbb{R}^{n}\right)} \sum_{j=0}^{\infty} 2^{-j M+j a} \sum_{k \in \mathbb{Z}^{n}} \frac{\inf _{y \in Q_{j k}}\left(\varphi_{j}^{*} f\right)_{a}(y)}{(1+|k|)^{n+M-a}} \\
& \lesssim\|h\|_{\mathcal{S}_{M+1}\left(\mathbb{R}^{n}\right)} \sum_{j=0}^{\infty} 2^{-j M+j a} \sum_{k \in \mathbb{Z}^{n}}(1+|k|)^{-(n+M-a)} \frac{\left\|\left(\varphi_{j}^{*} f\right)_{a}\right\|_{L^{p(\cdot)}\left(Q_{j k}\right)}}{\left\|\chi_{Q_{j k}}\right\|_{L^{p(\cdot)}\left(Q_{j k}\right)}} \\
& \lesssim\|f\|_{F_{p(\cdot), q(\cdot)}^{s(\cdot)}\left(\mathbb{R}^{n}\right)}\|h\|_{\mathcal{S}_{M+1}\left(\mathbb{R}^{n}\right)} \sum_{j=0}^{\infty} 2^{j\left(a-M-s_{-}\right)}
\end{aligned}
$$




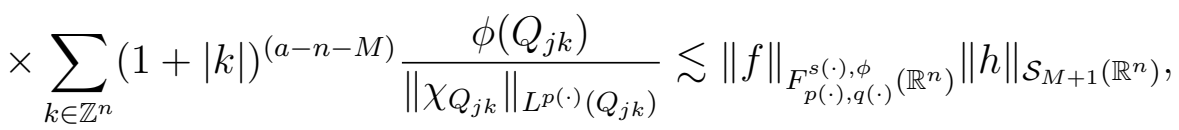

where $a$ is chosen as in (3.12). This finishes the proof of Proposition 3.20.

\section{A TRACE THEOREM}

In this section, we mainly establish a trace theorem for Triebel-Lizorkin-type spaces with variable exponents by applying the atomic characterization of these spaces obtained in Theorem 3.8.

To state our main result of this section, we first give some notation. For measurable functions $p, q, s$ and a set function $\phi$ being as in Definition 1.4, let $F_{p(\cdot, 0), q(\cdot, 0)}^{s(,, \widetilde{\phi})}\left(\mathbb{R}^{n-1}\right)$ denote the Triebel-Lizorkin-type spaces with variable exponents $p(\widetilde{\cdot}, 0), q(\widetilde{\cdot}, 0)$ and $s(\widetilde{\cdot}, 0)$ on $\mathbb{R}^{n-1} \times\{0\}$, where $\widetilde{\phi}$ is defined by setting, for all cubes $\widetilde{Q}$ of $\mathbb{R}^{n-1}, \widetilde{\phi}(\widetilde{Q}):=\phi\left(\widetilde{Q} \times[0, \ell(\widetilde{Q}))\right.$. In what follows, let $\mathbb{R}_{+}^{n}:=\mathbb{R}^{n-1} \times[0, \infty)$ and $\mathbb{R}_{-}^{n}:=\mathbb{R}^{n-1} \times(-\infty, 0]$.

Let $f \in F_{p(\cdot), q(\cdot)}^{s(\cdot),}\left(\mathbb{R}^{n}\right)$. Then, by Theorem 3.8, we have $f=\sum_{Q \in \mathcal{Q}^{*}} t_{Q} a_{Q}$ in $\mathcal{S}^{\prime}\left(\mathbb{R}^{n}\right)$ and

$$
\left\|\left\{t_{Q}\right\}_{Q \in \mathcal{Q}^{*}}\right\|_{f_{p(\cdot), q(\cdot)}^{s(\cdot),\left(\mathbb{R}^{n}\right)}} \leq C\|f\|_{F_{p(\cdot), q(\cdot)}^{s(\cdot),}\left(\mathbb{R}^{n}\right)},
$$

where $C$ is a positive constant independent of $f$ and, for each $Q \in \mathcal{Q}^{*}, a_{Q}$ is a smooth atom of $F_{p(\cdot), q(\cdot)}^{s(\cdot), \phi}\left(\mathbb{R}^{n}\right)$. Define the trace of $f$ by setting, for all $\widetilde{x} \in \mathbb{R}^{n-1}$,

$$
\operatorname{Tr}(f)(\widetilde{x}):=\sum_{Q \in \mathcal{Q}^{*}} t_{Q} a_{Q}(\widetilde{x}, 0) .
$$

This definition of $\operatorname{Tr}(f)$ is determined canonical for all $f \in F_{p(\cdot), q(\cdot)}^{s(\cdot)}\left(\mathbb{R}^{n}\right)$, since the actual construction of $a_{Q}$ in the proof of Theorem 3.8 implies that $t_{Q} a_{Q}$ is obtained canonically. Moreover, in Lemma 4.3 below, we show that the summation in (4.1) converges in $\mathcal{S}^{\prime}\left(\mathbb{R}^{n-1}\right)$. Thus, the trace operator is well defined.

The main result of this section is the following trace theorem.

Theorem 4.1. Let $n \geq 2, p, q \in \mathcal{P}\left(\mathbb{R}^{n}\right)$ satisfy

$$
0<p_{-} \leq p_{+}<\infty, \quad 0<q_{-} \leq q_{+}<\infty
$$

and $\frac{1}{p}, \frac{1}{q} \in C^{\log }\left(\mathbb{R}^{n}\right), s \in C_{\operatorname{loc}}^{\log }\left(\mathbb{R}^{n}\right) \cap L^{\infty}\left(\mathbb{R}^{n}\right)$ and $\phi$ be a set function satisfying the conditions $(\boldsymbol{S} \boldsymbol{1})$ and $(\boldsymbol{S} \mathscr{2})$. If

$$
s_{-}-\frac{1}{p_{-}}-(n-1)\left[\frac{1}{\min \left\{1, p_{-}\right\}}-1\right]>0,
$$

then

$$
\operatorname{Tr} F_{p(\cdot), q(\cdot)}^{s(\cdot), \phi}\left(\mathbb{R}^{n}\right)=F_{p(\cdot, 0), p(\cdot, 0)}^{s(\cdot, 0)-\frac{1}{p(r, 0)}, \widetilde{\phi}}\left(\mathbb{R}^{n-1}\right) .
$$

Remark 4.2. (i) When $p, q, s$ and $\phi$ are as in Remark 1.5(ii), Theorem 4.1 goes back to [80, Theorem 6.8]. Moreover, Theorem 4.1 coincides with the trace theorem for the classical Triebel-Lizorkin space $F_{p, q}^{s}\left(\mathbb{R}^{n}\right)$ with constant exponents (see [27, Theorem 11.1 and p. 134]) and, in this case, the condition (4.2) is optimal. 
(ii) In the case that $\phi$ is as in Remark 1.5(i), it was proved in [20, Theorem 3.13] (see also [56, Theorem 5.1(1)]) that the conclusion of Theorem 4.1 is true if $s$ and $p$ satisfy that, for all $x \in \mathbb{R}^{n}$,

$$
s(x)-\frac{1}{p(x)}-(n-1)\left[\frac{1}{\min \{1, p(x)\}}-1\right]>\delta
$$

for some $\delta \in(0, \infty)$, which is a little weaker than (4.2). The reason that the assumption (4.2), in this case, is a little stronger than that in [20, Theorem 3.13] (see also (4.3)) comes from an application of Theorem 3.8, which, in this case, can be further refined; see Remark 3.9(ii).

To prove Theorem 4.1, we first need to show that (4.1) converges in $\mathcal{S}^{\prime}\left(\mathbb{R}^{n-1}\right)$.

Lemma 4.3. Let $p, q, s$ and $\phi$ be as in Theorem 4.1 satisfying (4.2). Then, for all $f \in F_{p(\cdot), q(\cdot)}^{s(\cdot), \phi}\left(\mathbb{R}^{n}\right), \operatorname{Tr}(f) \in \mathcal{S}^{\prime}\left(\mathbb{R}^{n-1}\right)$.

Proof. Let $f \in F_{p(\cdot), q(\cdot)}^{s(\cdot), \phi}\left(\mathbb{R}^{n}\right)$. Then, by Theorem 3.8, we can write

$$
f=\sum_{Q \in \mathcal{Q}^{*}} t_{Q} a_{Q}
$$

in $\mathcal{S}^{\prime}\left(\mathbb{R}^{n}\right)$ and

$$
\left\|\left\{t_{Q}\right\}_{Q \in \mathcal{Q}^{*}}\right\|_{f_{p(\cdot), q(\cdot)}^{s(\cdot), \phi}\left(\mathbb{R}^{n}\right)} \lesssim\|f\|_{F_{p(\cdot), q(\cdot)}^{s(\cdot),}\left(\mathbb{R}^{n}\right)},
$$

where, for each $Q \in \mathcal{Q}^{*}, a_{Q}$ is a $(K, L)$-smooth atom supported near $Q$ of $F_{p(\cdot), q(\cdot)}^{s(\cdot),}\left(\mathbb{R}^{n}\right)$ with $K \in\left(s_{+}+\log _{2} \widetilde{c}_{1}, \infty\right)$ and $L$ is as in (3.1). Let

$$
A:=\left\{Q \in \mathcal{Q}^{*}: 3 \bar{Q} \cap\left\{\left(\widetilde{x}, x_{n}\right) \in \mathbb{R}^{n-1} \times \mathbb{R}: x_{n}=0\right\} \neq \emptyset\right\},
$$

where $\bar{Q}$ denotes the closure of $Q$ in $\mathbb{R}^{n}$. Since $\operatorname{supp} a_{Q} \subset 3 Q$ for all $Q \in \mathcal{Q}^{*}$, it follows that $a_{Q}(\cdot, 0)=0$ if $Q \notin A$. Observe that, if $Q_{j k} \in A$ with $j \in \mathbb{Z}_{+}$and $k:=\left(k_{1}, \ldots, k_{n}\right) \in \mathbb{Z}^{n}$, then $\left|k_{n}\right| \leq 2$. Therefore,

$$
\sum_{j=0}^{\infty} \sum_{k \in \mathbb{Z}^{n}} t_{Q_{j k}} a_{Q_{j k}}(\cdot, 0)=\sum_{j=0}^{\infty} \sum_{k \in \mathbb{Z}^{n},\left|k_{n}\right| \leq 2} t_{Q_{j k}} a_{Q_{j k}}(\cdot, 0) .
$$

Thus, to complete the proof of Lemma 4.3, it suffices to show that

$$
\lim _{N \rightarrow \infty, \Lambda \rightarrow \infty} \sum_{j=0}^{N} \sum_{\substack{k \in \mathbb{Z}^{n},\left|k_{n}\right| \leq 2 \\|k| \leq \Lambda}} t_{Q_{j k}} a_{Q_{j k}}(\widetilde{r}, 0)
$$

exists in $\mathcal{S}^{\prime}\left(\mathbb{R}^{n-1}\right)$. By (4.2), we see that

$$
\begin{aligned}
& s_{-}-\frac{n}{p_{-}}+\frac{\min \left\{1, p_{-}\right\}}{p_{-}}(n-1) \\
& =s_{-}-\frac{1}{p_{-}}-\frac{n-1}{p_{-}}\left(1-\min \left\{1, p_{-}\right\}\right) \\
& \geq s_{-}-\frac{1}{p_{-}}-\frac{n-1}{\min \left\{1, p_{-}\right\}}\left(1-\min \left\{1, p_{-}\right\}\right)
\end{aligned}
$$




$$
=s_{-}-\frac{1}{p_{-}}-(n-1)\left[\frac{1}{\min \left\{1, p_{-}\right\}}-1\right]>0,
$$

which implies that there exists $r \in\left(0, \min \left\{1, p_{-}\right\}\right)$such that

$$
s_{-}-\frac{n}{p_{-}}+\frac{r}{p_{-}}(n-1)>0 .
$$

Let $\widetilde{p}(\cdot)$ and $\widetilde{s}(\cdot)$ be as in the proof of Theorem 3.8. Then $\widetilde{s}_{-}-\frac{r}{p_{-}}>0$. For all $j \in \mathbb{Z}_{+}$and $k \in \mathbb{Z}^{n}$, let

$$
\Delta_{k, j}:=\mathbb{R}^{n-1} \times\left[k_{n} 2^{-j},\left(k_{n}+1\right) 2^{-j}\right) .
$$

Then, by the smoothness condition (A3), we know that, for all $h \in \mathcal{S}\left(\mathbb{R}^{n-1}\right)$ and $j \in \mathbb{Z}_{+}$,

$$
\begin{aligned}
\mathrm{I} & :=\left|\left\langle\sum_{\substack{k \in \mathbb{Z}^{n},\left|k_{n}\right| \leq 2 \\
|k| \leq \Lambda}} t_{Q_{j k}} a_{Q_{j k}}(\widetilde{\cdot}, 0), h(\cdot)\right\rangle\right| \\
& \lesssim 2^{j n / 2} \sum_{\substack{k \in \mathbb{Z}^{n},\left|k_{n}\right| \leq 2 \\
|k| \leq \Lambda}}\left|t_{Q_{j k}}\right| \int_{\mathbb{R}^{n-1}} \frac{(1+|\widetilde{y}|)^{-\delta}}{\left(1+2^{j}\left|(\widetilde{y}, 0)-x_{Q_{j k}}\right|\right)^{R}} d \widetilde{y} \\
& \sim 2^{j n / 2+j} \sum_{\substack{k \in \mathbb{Z}^{n},\left|k_{n}\right| \leq 2 \\
|k| \leq \Lambda}}\left|t_{Q_{j k}}\right| \int_{\Delta_{k, j}} \frac{(1+|\widetilde{y}|)^{-\delta}}{\left(1+2^{j}\left|(\widetilde{y}, 0)-x_{Q_{j k}}\right|\right)^{R}} d \widetilde{y} d y_{n} \\
& \lesssim 2^{j n / 2+j} \sum_{\substack{k \in \mathbb{Z}^{n},\left|k_{n}\right| \leq 2 \\
|k| \leq \Lambda}}\left|t_{Q_{j k}}\right| \int_{\mathbb{R}^{n}} \frac{(1+|y|)^{-\delta} \chi_{\Delta_{k, j}}(y)}{\left(1+2^{j}\left|y-x_{Q_{j k}}\right|\right)^{R}} d y,
\end{aligned}
$$

where $R \in(0, \infty)$ is chosen large enough, and $\delta \in(0, \infty)$ will be determined later. By an argument similar to that used in the proof of Theorem 3.8, we find that

$$
\mathrm{I} \lesssim 2^{-j\left(\widetilde{s}_{-}-1\right)}\|t\|_{f_{p(\cdot), q(\cdot)}^{s(\cdot),}\left(\mathbb{R}^{n}\right)}\left\|\frac{\chi_{\Delta_{j}}}{(1+|\cdot|)^{\delta-\delta_{0}}}\right\|_{L^{(\widetilde{p}(\cdot))^{*}\left(\mathbb{R}^{n}\right)}},
$$

where $\Delta_{j}:=\mathbb{R}^{n-1} \times\left[-2^{-j+1}, 2^{-j+1}\right]$. On the other hand, by choosing $\delta$ large enough, we see that

$$
\begin{aligned}
& \int_{\mathbb{R}^{n}}\left[\frac{\chi_{\Delta_{j}}(y) /(1+|y|)^{-\delta+\delta_{0}}}{2^{-j /\left(\widetilde{p}(\cdot)^{*}\right)_{+}}}\right]^{(\widetilde{p}(y))^{*}} d y \\
& \lesssim 2^{j} \int_{\mathbb{R}^{n-1} \times\left[-2^{-j+1}, 2^{-j+1}\right]}\left[\frac{1}{(1+|\widetilde{y}|)^{\delta-\delta_{0}}}\right]^{\left(\widetilde{p}(\cdot)^{*}\right)_{-}} d \widetilde{y} d y_{n} \\
& \lesssim \int_{\mathbb{R}^{n-1}}\left[\frac{1}{(1+|\widetilde{y}|)^{\delta-\delta_{0}}}\right]^{\left(\widetilde{p}(\cdot)^{*}\right)-} d \widetilde{y} \lesssim 1,
\end{aligned}
$$

which, together with Remark 1.1(ii), implies that

$$
\left\|\frac{\chi_{\Delta_{j}}}{(1+|\cdot|)^{\delta-\delta_{0}}}\right\|_{L^{(\widetilde{p}(\cdot))^{*}\left(\mathbb{R}^{n}\right)}} \lesssim 2^{-j /\left(\widetilde{p}(\cdot)^{*}\right)_{+}} \sim 2^{-j\left(1-\frac{r}{p_{-}}\right)} .
$$


From this and (4.5), we deduce that $\mathrm{I} \lesssim 2^{-j\left(\widetilde{s}_{-}-\frac{r}{p_{-}}\right)}\|t\|_{f_{p(\cdot), q(\cdot)}^{s(\cdot),}\left(\mathbb{R}^{n}\right)}$, which, combined with the fact that $\widetilde{s}_{-}-\frac{r}{p_{-}}>0$, implies that (4.4) converges in $\mathcal{S}^{\prime}\left(\mathbb{R}^{n-1}\right)$. This finishes the proof of Lemma 4.3.

Next we prove Theorem 4.1 by beginning with several technical lemmas.

Lemma 4.4. Let $p_{i}, q_{i}, s_{i}$ and $\phi$ be as in Definition 1.4 with $p, q$, s replaced by $p_{i}, q_{i}, s_{i}$, respectively, where $i \in\{1,2\}$. If $s_{1} \leq s_{2}, p_{1} \leq p_{2},\left(p_{1}\right)_{\infty}=\left(p_{2}\right)_{\infty}$ and $q_{1} \geq q_{2}$, then

$$
F_{p_{2}(\cdot), q_{2}(\cdot)}^{s_{2}(\cdot),}\left(\mathbb{R}^{n}\right) \hookrightarrow F_{p_{1}(\cdot), q_{1}(\cdot)}^{s_{1}(\cdot),}\left(\mathbb{R}^{n}\right) .
$$

Proof. By [20, Proposition 6.5], we find that

$$
L^{p_{2}(\cdot)}\left(\mathbb{R}^{n}\right) \hookrightarrow L^{p_{1}(\cdot)}\left(\mathbb{R}^{n}\right) .
$$

From this, $s_{1} \leq s_{2}$ and (2.6), we can easily deduce the desired conclusion, the details being omitted. This finishes the proof of Lemma 4.4.

Lemma 4.5. Let $p_{i}, q_{i}, s_{i}$ and $\phi$ be as in Theorem 4.1 with $p, q$, s replaced by $p_{i}, q_{i}, s_{i}$, where $i \in\{1,2\}$. Assume that $s_{1}=s_{2}$ and $p_{1}=p_{2}$ on $\mathbb{R}_{+}^{n}$ or $\mathbb{R}_{-}^{n}$, and that $s_{1} \leq s_{2}$ and $p_{1} \leq p_{2}$. If

$$
\left(s_{2}\right)_{-}-\frac{1}{\left(p_{2}\right)_{-}}-(n-1)\left[\frac{1}{\min \left\{1,\left(p_{2}\right)_{-}\right\}-1}\right]>0,
$$

then

$$
\operatorname{Tr} F_{p_{1}(\cdot), q_{1}(\cdot)}^{s_{1}(\cdot)}\left(\mathbb{R}^{n}\right)=\operatorname{Tr} F_{p_{2}(\cdot), q_{2}(\cdot)}^{s_{2}(\cdot),}\left(\mathbb{R}^{n}\right) ;
$$

moreover, if $q(\cdot)$ is as in Theorem 4.1, then

$$
\operatorname{Tr} F_{p_{1}(\cdot), q_{1}(\cdot)}^{s_{1}(\cdot)}\left(\mathbb{R}^{n}\right)=\operatorname{Tr} F_{p_{1}(\cdot), q(\cdot)}^{s_{1}(\cdot),}\left(\mathbb{R}^{n}\right) .
$$

To prove Lemma 4.5, we need the following conclusion.

Proposition 4.6. Let $p, q, s, \phi$ be as in Definition 1.4 and $\delta \in(0,1)$. Suppose that, for each $Q \in \mathcal{Q}^{*}, E_{Q} \subset 3 Q$ is a measurable set with $\left|E_{Q}\right| \geq \delta|Q|$. Then, for all $t:=\left\{t_{Q}\right\}_{Q \in \mathcal{Q}^{*}} \subset \mathbb{C}, t \in f_{p(\cdot), q(\cdot)}^{s(\cdot),}\left(\mathbb{R}^{n}\right)$ if and only if $\|t\|_{f_{p(\cdot), q(\cdot)}^{s(\cdot),}\left(\mathbb{R}^{n}\right)}<\infty$, where

$$
\begin{aligned}
& \|t\|_{f_{p(\cdot), q(\cdot)}^{s(\cdot),}\left(\mathbb{R}^{n}\right)} \\
& :=\sup _{P \in \mathcal{Q}} \frac{1}{\phi(P)}\left\|\left\{\sum_{j=\left(j_{P} \vee 0\right)}^{\infty} \sum_{\ell(Q)=2^{-j}}\left[2^{j s(\cdot)}\left|t_{Q}\right||Q|^{-\frac{1}{2}} \chi_{E_{Q}}\right]^{q(\cdot)}\right\}^{\frac{1}{q(\cdot)}}\right\|_{L^{p(\cdot)}(P)} .
\end{aligned}
$$

Proof. We first suppose that $\|t\|_{f_{p(\cdot), q(\cdot)}^{s(\cdot),}\left(\mathbb{R}^{n}\right)}<\infty$ and show that $t \in f_{p(\cdot), q(\cdot)}^{s(\cdot),}\left(\mathbb{R}^{n}\right)$.

Notice that, for all $m \in(n, \infty), Q \in \mathcal{Q}^{*}$ and $x \in Q$,

$$
\chi_{Q}(x) \lesssim \eta_{j_{Q}, m+C_{\log }(s)} * \chi_{E_{Q}}(x) .
$$

From this, Lemmas 2.9 and 2.10, we deduce that

$$
\|t\|_{f_{p(\cdot), q(\cdot)}^{s(\cdot), \phi}\left(\mathbb{R}^{n}\right)}
$$




$$
\begin{aligned}
& =\sup _{P \in \mathcal{Q}} \frac{1}{\phi(P)}\left\|\left\{\sum_{j=\left(j_{P} \vee 0\right)}^{\infty} \sum_{\ell(Q)=2^{-j}}\left[2^{j r s(\cdot)}\left|t_{Q}\right|^{r}|Q|^{-\frac{r}{2}} \chi_{Q}\right]^{\frac{q(\cdot)}{r}}\right\}^{\frac{1}{q(\cdot)}}\right\|_{L^{p(\cdot)}(P)} \\
& \lesssim \sup _{P \in \mathcal{Q}} \frac{1}{\phi(P)} \|\left\{\sum_{j=\left(j_{P} \vee 0\right)}^{\infty}\left[\eta_{j, m} *\left(2^{j r s(\cdot)} \sum_{\substack{Q \subset P \\
\ell(Q)=2^{-j}}}\left|t_{Q}\right|^{r} \frac{\chi_{E_{Q}}}{|Q|^{\frac{r}{2}}}\right]^{\frac{q(\cdot)}{r}}\right\}^{\frac{1}{q(\cdot)}} \|_{L^{p(\cdot)}(P)}\right. \\
& \lesssim \sup _{P \in \mathcal{Q}} \frac{1}{\phi(P)}\left\|\left\{\sum_{j=\left(j_{P} \vee 0\right)}^{\infty}\left[2^{j s(\cdot)} \sum_{\substack{Q \subset P \\
\ell(Q)=2^{-j}}}\left|t_{Q} \| Q\right|^{-\frac{1}{2}} \chi_{E_{Q}}\right]^{q(\cdot)}\right\}^{\frac{1}{q(\cdot)}}\right\|_{L^{p(\cdot)}(P)} \\
& \sim\|t\|_{f_{p(\cdot), q(\cdot)}^{s(\cdot), \phi}\left(\mathbb{R}^{n}\right)},
\end{aligned}
$$

which implies that $t \in f_{p(\cdot), q(\cdot)}^{s(\cdot), \phi}\left(\mathbb{R}^{n}\right)$.

Conversely, by an argument similar to the above and the fact that, for all $m \in(n, \infty), Q \in \mathcal{Q}^{*}$ and $x \in E_{Q}, \chi_{E_{Q}}(x) \lesssim \eta_{j_{Q}, m+C_{\log (s)}} * \chi_{Q}(x)$, we conclude that, for all $t \in f_{p(\cdot), q(\cdot)}^{s(\cdot), \phi}\left(\mathbb{R}^{n}\right),\|t\|_{f_{p(\cdot), q(\cdot)}^{s(\cdot),}\left(\mathbb{R}^{n}\right)} \lesssim\|t\|_{f_{p(\cdot), q(\cdot)}^{s(\cdot), \phi}\left(\mathbb{R}^{n}\right)}$. This finishes the proof of Proposition 4.6.

Proof of Lemma 4.5. From Remark 1.2(i) and the condition that $p_{1}=p_{2}$ on $\mathbb{R}_{+}^{n}$ or $\mathbb{R}_{-}^{n}$, we deduce that $\left(p_{1}\right)_{\infty}=\left(p_{2}\right)_{\infty}$. Let $r_{0}:=\min \left\{\left(q_{2}\right)_{-},\left(q_{1}\right)_{-}\right\}$and $r_{1}:=\max \left\{\left(q_{2}\right)_{+},\left(q_{1}\right)_{+}\right\}$. Then, by Lemma 4.4 and (2.6), we see that

$$
F_{p_{2}(\cdot), r_{0}}^{s_{2}(\cdot),}\left(\mathbb{R}^{n}\right) \hookrightarrow F_{p_{1}(\cdot), q_{1}(\cdot)}^{s_{1}(\cdot), \phi}\left(\mathbb{R}^{n}\right) \hookrightarrow F_{p_{1}(\cdot), r_{1}}^{s_{1}(\cdot),}\left(\mathbb{R}^{n}\right)
$$

and

$$
F_{p_{2}(\cdot), r_{0}}^{s_{2}(\cdot), \phi}\left(\mathbb{R}^{n}\right) \hookrightarrow F_{p_{2}(\cdot), q_{2}(\cdot)}^{s_{2}(\cdot), \phi}\left(\mathbb{R}^{n}\right) \hookrightarrow F_{p_{1}(\cdot), r_{1}}^{s_{1}(\cdot),}\left(\mathbb{R}^{n}\right) .
$$

By Lemma 4.3 and an argument similar to that used in the proof of [20, Lemma 7.2], with [20, Theorem 3.8 and Lemma 7.1] replaced by Theorem 3.8 and Proposition 4.6, we conclude that, for all $f \in F_{p_{1}(\cdot), r_{1}}^{s_{1}(\cdot),}\left(\mathbb{R}^{n}\right), \operatorname{Tr}(f)$ exists in $\mathcal{S}^{\prime}\left(\mathbb{R}^{n-1}\right)$ and $\operatorname{Tr} F_{p_{1}(\cdot), r_{1}}^{s_{1}(\cdot), \phi}\left(\mathbb{R}^{n}\right) \subset \operatorname{Tr} F_{p_{2}(\cdot), r_{0}}^{s_{2}(\cdot), \phi}\left(\mathbb{R}^{n}\right)$. From this, (4.7) and (4.8), we deduce that

$$
\begin{aligned}
\operatorname{Tr} F_{p_{1}(\cdot), q_{1}(\cdot)}^{s_{1}(\cdot), \phi}\left(\mathbb{R}^{n}\right) & \subset \operatorname{Tr} F_{p_{1}(\cdot), r_{1}}^{s_{1}(\cdot),}\left(\mathbb{R}^{n}\right) \subset \operatorname{Tr} F_{p_{2}(\cdot), r_{0}}^{s_{2}(\cdot),}\left(\mathbb{R}^{n}\right) \subset \operatorname{Tr} F_{p_{2}(\cdot), q_{2}(\cdot)}^{s_{2}(\cdot),}\left(\mathbb{R}^{n}\right) \\
& \subset \operatorname{Tr} F_{p_{1}(\cdot), r_{1}}^{s_{1}(\cdot),}\left(\mathbb{R}^{n}\right) \subset \operatorname{Tr} F_{p_{2}(\cdot), r_{0}}^{s_{2}(\cdot),}\left(\mathbb{R}^{n}\right) \subset \operatorname{Tr} F_{p_{1}(\cdot), q_{1}(\cdot)}^{s_{1}(\cdot),}\left(\mathbb{R}^{n}\right),
\end{aligned}
$$

which completes the proof of Lemma 4.5.

Remark 4.7. By the proof of Lemma 4.5, we see that the condition (4.6) is only used to ensure that $\operatorname{Tr} F_{p_{2}(\cdot), q_{2}(\cdot)}^{s_{2}(\cdot),}\left(\mathbb{R}^{n}\right)$ exists in $\mathcal{S}^{\prime}\left(\mathbb{R}^{n-1}\right)$. Thus, by an argument similar to that used in the proof of Lemma 4.5, we have the following conclusion, the details being omitted. Under the same assumption as in Lemma 4.5, if, for all $f \in F_{p_{2}(\cdot), q_{2}(\cdot)}^{s_{2}(\cdot),}\left(\mathbb{R}^{n}\right)$, the trace of $f$ defined as in $(4.1)$ exists in $\mathcal{S}^{\prime}\left(\mathbb{R}^{n-1}\right)$, then, 
for all $g \in F_{p_{1}(\cdot), q_{1}(\cdot)}^{s_{1}(\cdot), \phi}\left(\mathbb{R}^{n}\right)$, the trace of $g$ defined as in (4.1) also exists in $\mathcal{S}^{\prime}\left(\mathbb{R}^{n-1}\right)$; moreover,

$$
\operatorname{Tr} F_{p_{1}(\cdot), q_{1}(\cdot)}^{s_{1}(\cdot),}\left(\mathbb{R}^{n}\right)=\operatorname{Tr} F_{p_{2}(\cdot), q_{2}(\cdot)}^{s_{2}(\cdot),}\left(\mathbb{R}^{n}\right) .
$$

Lemma 4.8. Let $p_{i}, q, s_{i}$ be as in Theorem 4.1 with $p$ and $s$ replaced by $p_{i}$ and $s_{i}$, $i \in\{1,2\}$. Assume that $s_{1}(x)=s_{2}(x)$ and $p_{1}(x)=p_{2}(x)$ for all $x \in \mathbb{R}^{n-1} \times\{0\}$. If (4.2) is satisfied with $(s, p)$ replaced, respectively, by $\left(s_{1}, p_{1}\right)$ and $\left(s_{2}, p_{2}\right)$, then

$$
\operatorname{Tr} F_{p_{1}(\cdot), q(\cdot)}^{s_{1}(\cdot),}\left(\mathbb{R}^{n}\right)=\operatorname{Tr} F_{p_{2}(\cdot), p_{2}(\cdot)}^{s_{2}(\cdot),}\left(\mathbb{R}^{n}\right) .
$$

Proof. For all $x \in \mathbb{R}^{n}$ and $i \in\{1,2\}$, let $\widetilde{s}_{i}(x):=s_{i}(x)$ if $x \in \mathbb{R}_{-}^{n}$ and $\widetilde{s}_{i}(x):=$ $\min \left\{s_{1}(x), s_{2}(x)\right\}$ otherwise, and, for all $x \in \mathbb{R}^{n}, \widetilde{s}(x):=\min \left\{s_{1}(x), s_{2}(x)\right\}$. Similarly, for $i \in\{1,2\}$, let $\widetilde{p}_{i}(x):=p_{i}(x)$ if $x \in \mathbb{R}_{-}^{n}$ and

$$
\widetilde{p}_{i}(x):=\min \left\{p_{1}(x), p_{2}(x)\right\}
$$

otherwise, and, for all $x \in \mathbb{R}^{n}$,

$$
\widetilde{p}(x):=\min \left\{p_{1}(x), p_{2}(x)\right\} .
$$

Then, by applying Lemma 4.5 and Remark 4.7, we conclude that

$$
\begin{aligned}
\operatorname{Tr} F_{p_{1}(\cdot), q(\cdot)}^{s_{1}(\cdot), \phi}\left(\mathbb{R}^{n}\right) & =\operatorname{Tr} F_{p_{1}(\cdot), p_{1}(\cdot)}^{s_{1}(\cdot), \phi}\left(\mathbb{R}^{n}\right)=\operatorname{Tr} F_{\widetilde{p}_{1}(\cdot), \widetilde{p}_{1}(\cdot)}^{\widetilde{s}_{1}(\cdot),}\left(\mathbb{R}^{n}\right) \\
& =\operatorname{Tr} F_{\widetilde{p}(\cdot), \widetilde{p}(\cdot)}^{\widetilde{s}(\cdot)}\left(\mathbb{R}^{n}\right)=\operatorname{Tr} F_{\widetilde{p}_{2}(\cdot), \widetilde{p}_{2}(\cdot)}^{\widetilde{s}_{2}(\cdot)}\left(\mathbb{R}^{n}\right)=\operatorname{Tr} F_{p_{2}(\cdot), p_{2}(\cdot)}^{s_{2}(\cdot),}\left(\mathbb{R}^{n}\right),
\end{aligned}
$$

which completes the proof of Lemma 4.8.

In what follows, let $\mathcal{Q}\left(\mathbb{R}^{n}\right):=\mathcal{Q}$ and $\mathcal{Q}^{*}\left(\mathbb{R}^{n}\right):=\mathcal{Q}^{*}$. Denote by $\mathcal{Q}\left(\mathbb{R}^{n-1}\right)$ the set of all dyadic cubes of $\mathbb{R}^{n-1}$ and $\mathcal{Q}^{*}\left(\mathbb{R}^{n-1}\right)$ the set of all dyadic cubes $\widetilde{Q}$ of $\mathbb{R}^{n-1}$ with $\ell(\widetilde{Q}) \leq 1$.

Proof of Theorem 4.1. By Lemma 4.8, we may assume that $q=p$ with $p$ and $s$ independent of the $n$-th coordinate $x_{n}$ with $\left|x_{n}\right| \leq 2$. Indeed, let, for all $\left(\widetilde{x}, x_{n}\right) \in \mathbb{R}^{n-1} \times[-2,2], \widetilde{p}_{0}\left(\widetilde{x}, x_{n}\right):=p(\widetilde{x}, 0)$. Then $\widetilde{p}_{0} \in C^{\log }\left(\mathbb{R}^{n-1} \times[-2,2]\right)$. By [19, Proposition 4.1.7], we find that $\widetilde{p}_{0}$ has an extension $\widetilde{p} \in C^{\log }\left(\mathbb{R}^{n}\right)$ with $\widetilde{p}_{-}=\left(\widetilde{p}_{0}\right)_{-}$and $\widetilde{p}_{\infty}=\left(\widetilde{p}_{0}\right)_{\infty}$. Define $\widetilde{s}$ by setting, for all $\left(\widetilde{x}, x_{n}\right) \in \mathbb{R}^{n-1} \times \mathbb{R}$, $\widetilde{s}\left(\widetilde{x}, x_{n}\right):=s(\widetilde{x}, 0)$. Then it is easy to see that $\widetilde{s} \in C_{\operatorname{loc}}^{\log }\left(\mathbb{R}^{n}\right) \cap L^{\infty}\left(\mathbb{R}^{n}\right)$. Moreover, $\widetilde{p}$ and $\widetilde{s}$ are independent of the $n$-th coordinate $x_{n}$ with $\left|x_{n}\right| \leq 2$, and satisfy

$$
\widetilde{s}_{-}-\frac{1}{\widetilde{p}_{-}}-(n-1)\left[\frac{1}{\min \left\{1, \widetilde{p}_{-}\right\}}-1\right]>0 \text {. }
$$

Then, by Lemma 4.8, we see that

$$
\operatorname{Tr} F_{p(\cdot), q(\cdot)}^{s(\cdot),}\left(\mathbb{R}^{n}\right)=\operatorname{Tr} F_{\widetilde{p}(\cdot), \widetilde{p}(\cdot)}^{\widetilde{s}(\cdot), \phi}\left(\mathbb{R}^{n}\right) .
$$

For notational simplicity, let, for all $\widetilde{x} \in \mathbb{R}^{n-1}$,

and

$$
\begin{gathered}
\beta(\widetilde{x}, 0):=s(\widetilde{x}, 0)-\frac{1}{p(\widetilde{x}, 0)}, \\
F_{p(r, 0)}^{\beta(r, 0), \widetilde{\phi}}\left(\mathbb{R}^{n-1}\right):=F_{p(\cdot, 0), p(\cdot, 0)}^{\beta(\cdot, 0), \widetilde{\phi}}\left(\mathbb{R}^{n-1}\right)
\end{gathered}
$$

$$
f_{p(\cdot, 0)}^{\beta(\cdot, 0), \widetilde{\phi}}\left(\mathbb{R}^{n-1}\right):=f_{p(\cdot, 0), p(\cdot, 0)}^{\beta(\cdot, 0), \widetilde{\phi}}\left(\mathbb{R}^{n-1}\right) .
$$


We finish the proof of Theorem 4.1 by two steps.

Step 1) We show that, for all $f \in F_{p(\cdot), q(\cdot)}^{s(\cdot),}\left(\mathbb{R}^{n}\right), \operatorname{Tr}(f) \in \mathcal{S}^{\prime}\left(\mathbb{R}^{n-1}\right)$ and

$$
\|\operatorname{Tr}(f)\|_{F_{p(\cdot, 0)}^{\beta(\cdot, 0), \widetilde{\phi}}\left(\mathbb{R}^{n-1}\right)} \lesssim\|f\|_{F_{p(\cdot), p(\cdot)}^{s(\cdot), \phi}\left(\mathbb{R}^{n}\right)} .
$$

Without loss of generality, we may assume that $\|f\|_{F_{p(\cdot), p(\cdot)}^{s(\cdot),}\left(\mathbb{R}^{n}\right)}=1$. By Theorem 3.8 , we see that

$$
f=\sum_{Q \in \mathcal{Q}^{*}\left(\mathbb{R}^{n}\right)} t_{Q} a_{Q}
$$

in $\mathcal{S}^{\prime}\left(\mathbb{R}^{n}\right)$, where, for all $Q \in \mathcal{Q}^{*}, a_{Q}$ is a $(K, L)$-smooth atom supported near $Q$ of $F_{p(\cdot), p(\cdot)}^{s(\cdot), \phi}\left(\mathbb{R}^{n}\right)$ with

$$
K \in\left(s_{+}+\max \left\{0, \log _{2} \widetilde{c}_{1}\right\}, \infty\right) \text { and } L \in\left(\frac{n}{\min \left\{1, p_{-}\right\}}-n-s_{-}, \infty\right)
$$

and $t:=\left\{t_{Q}\right\}_{Q \in \mathcal{Q}^{*}\left(\mathbb{R}^{n}\right)} \in f_{p(\cdot), p(\cdot)}^{s(\cdot),}\left(\mathbb{R}^{n}\right)$, which can be chosen such that

$$
\|t\|_{f_{p(\cdot), p(\cdot)}^{s(\cdot),}\left(\mathbb{R}^{n}\right)} \lesssim\|f\|_{F_{p(\cdot), p(\cdot)}^{s(\cdot),}\left(\mathbb{R}^{n}\right)} \cdot
$$

Since $\operatorname{supp} a_{Q} \subset 3 Q$, it follows that, if $i \notin\{0,1,2\}$, then

$$
a_{\widetilde{Q} \times[(i-1) \ell(\widetilde{Q}), i \ell(\widetilde{Q}))}(\widetilde{\cdot}, 0)=0,
$$

which implies that $\operatorname{Tr}(f)$ can be rewritten as

$$
\sum_{i=0}^{2} \sum_{\widetilde{Q} \in \mathcal{Q}^{*}\left(\mathbb{R}^{n-1}\right)} t_{\widetilde{Q} \times[(i-1) \ell(\widetilde{Q}), i \ell(\widetilde{Q}))} a_{\widetilde{Q} \times[(i-1) \ell(\widetilde{Q}), i \ell(\widetilde{Q}))}(\cdot, 0) .
$$

Therefore, to show (4.9), by Theorem 3.8 again, it can be reduced to prove that each

$$
b_{\widetilde{Q}}^{(i)}:=[\ell(\widetilde{Q})]^{\frac{1}{2}} a_{\widetilde{Q} \times[(i-1) \ell(\widetilde{Q}), i \ell(\widetilde{Q}))}
$$

is a $(\widetilde{K}, \widetilde{L})$-smooth atom supported near $\widetilde{Q}$ of $F_{p(r, 0)}^{\beta(r, 0), \widetilde{\phi}}\left(\mathbb{R}^{n-1}\right)$ with

$$
\begin{gathered}
\widetilde{K} \in\left((s(\widetilde{\cdot}, 0))_{+}+\max \left\{0, \log _{2} \widetilde{c}_{1}\right\}, \infty\right), \\
\widetilde{L} \in\left(\frac{n-1}{\min \left\{1,(p(\cdot, 0))_{-}\right\}}-(n-1)-(s(\widetilde{\cdot}, 0))_{-}, \infty\right)
\end{gathered}
$$

and

$$
\left\|\{\lambda \overbrace{\widetilde{Q}}^{(i)}\}_{\widetilde{Q} \in \mathcal{Q}^{*}\left(\mathbb{R}^{n-1}\right)}\right\|_{f_{p(r, 0)}^{\beta(,, 0), \widetilde{\phi}}\left(\mathbb{R}^{n-1}\right)}<\infty
$$

where, for all $\widetilde{Q} \in \mathcal{Q}^{*}\left(\mathbb{R}^{n-1}\right)$,

$$
\lambda_{\widetilde{Q}}^{(i)}:=[\ell(\widetilde{Q})]^{-\frac{1}{2}} t_{\widetilde{Q} \times[(i-1) \ell(\widetilde{Q}), i \ell(\widetilde{Q}))} .
$$

By (4.2), we see that

$$
\frac{n-1}{\min \left\{1,(p(\widetilde{r}, 0))_{-}\right\}}-(n-1)-(s(\cdot, 0))_{-}<0
$$


and then, by Remark 3.7(i), we know that the vanishing moment for $(\widetilde{K}, \widetilde{L})$ smooth atoms of $F_{p(r, 0)}^{\beta(\cdot, 0), \widetilde{\phi}}\left(\mathbb{R}^{n-1}\right)$ is void. Since $\operatorname{supp} a_{Q} \subset 3 Q, \widetilde{K} \leq K$ and, for all $\alpha \in \mathbb{Z}_{+}^{n}$, with $|\alpha| \leq K$, and all $x \in \mathbb{R}^{n},\left|D^{\alpha} a_{Q}(x)\right| \leq 2^{(|\alpha|+n / 2) j}$, it follows that, for $i \in\{0,1,2\}, \widetilde{\alpha} \in \mathbb{Z}_{+}^{n}$, with $|\widetilde{\alpha}| \leq \widetilde{K}$, and all $x \in \mathbb{R}^{n}$,

$$
\left|D^{\widetilde{\alpha}} b_{Q}^{(i)}(x)\right| \leq 2^{(|\widetilde{\alpha}|+n / 2) j}
$$

and $\operatorname{supp} b_{Q}^{(i)} \subset 3 \widetilde{Q}$. Thus, for $i \in\{0,1,2\}, b_{\widetilde{Q}}^{(i)}$ is a $(\widetilde{K}, \widetilde{L})$-smooth atom supported near $\widetilde{Q}$ of $F_{p(\cdot, 0)}^{\beta(\cdot, 0), \widetilde{\phi}}\left(\mathbb{R}^{n-1}\right)$ with $(\widetilde{K}, \widetilde{L})$ as in (4.12) and (4.13).

Let $\lambda^{(i)}:=\left\{\lambda_{\widetilde{Q}}^{(i)}\right\}_{\widetilde{Q} \in \mathcal{Q}^{*}\left(\mathbb{R}^{n-1}\right)}$, where $i \in\{0,1,2\}$. Next we show that, for any given dyadic cube $\widetilde{P} \subset \mathbb{R}^{n-1}$,

$$
\frac{1}{\widetilde{\phi}(\widetilde{P})}\left\|\left\{\sum_{j=\left(j_{\widetilde{P}} \vee 0\right)}^{\infty} \sum_{\substack{\widetilde{Q} \in \mathcal{Q}^{*}\left(\mathbb{R}^{n-1}\right) \\ \ell(\widetilde{Q})=2^{-j}}}\left[2^{j(\beta(\cdot, 0))}\left|\lambda_{\widetilde{Q}}^{(i)} \| \widetilde{Q}\right|^{-\frac{1}{2}} \chi_{\widetilde{Q}}\right]^{p(\cdot, 0)}\right\}^{\frac{1}{p(\cdot, 0)}}\right\|_{L^{p(\cdot, 0)}(\widetilde{P})}
$$

is finite. By $\|f\|_{F_{p(\cdot), p(\cdot)}^{s(\cdot),}\left(\mathbb{R}^{n}\right)}=1$ and $(4.11)$, we see that there exists a positive constant $C_{0}$ such that, for all $P \in \mathcal{Q}\left(\mathbb{R}^{n}\right)$,

$$
\frac{1}{\phi(P)}\left\|\left\{\sum_{j=\left(j_{P} \vee 0\right)}^{\infty} \sum_{\substack{Q \in \mathcal{Q}^{*}\left(\mathbb{R}^{n}\right) \\ \ell(Q)=2^{-j}}}\left[2^{j s(\cdot)}\left|t_{Q} \| Q\right|^{-\frac{1}{2}} \chi_{Q}\right]^{p(\cdot)}\right\}^{\frac{1}{p(\cdot)}}\right\|_{L^{p(\cdot)}(P)} \leq C_{0},
$$

which, together with Remark 1.1(ii), implies that, for all $P \in \mathcal{Q}\left(\mathbb{R}^{n}\right)$,

$$
\int_{\mathbb{R}^{n}}\left\{\sum_{j=\left(j_{P} \vee 0\right)}^{\infty} \sum_{\substack{Q \in \mathcal{Q}^{*}\left(\mathbb{R}^{n}\right) \\ \ell(Q)=2^{-j}}}\left[2^{j s(\cdot)}\left|t_{Q}\right||Q|^{-\frac{1}{2}} \frac{\chi_{P}}{C_{0} \phi(P)} \chi_{Q}\right]^{p(\cdot)}\right\} d x \leq 1 .
$$

On the other hand, for all dyadic cube $\widetilde{P} \in \mathcal{Q}\left(\mathbb{R}^{n-1}\right)$, we have

$$
\begin{aligned}
\mathrm{I}(\widetilde{P}) & :=\int_{\mathbb{R}^{n-1}} \sum_{j=\left(j_{\widetilde{P}} \vee 0\right)}^{\infty} \sum_{\substack{\widetilde{Q} \in \mathcal{Q}\left(\mathbb{R}^{n-1}\right) \\
\ell(\widetilde{Q})=2^{-j}}}\left[2^{j \beta(\widetilde{x}, 0)} \frac{\left|\lambda_{\widetilde{Q}}^{(i)}\right|}{|\widetilde{Q}|^{\frac{1}{2}}} \frac{\chi_{\widetilde{P}}(\widetilde{x}) \chi_{\widetilde{Q}}(\widetilde{x})}{C_{0} \widetilde{\phi}(\widetilde{P})}\right]^{p(\widetilde{x}, 0)} d \widetilde{x} \\
& =\sum_{j=\left(j_{\widetilde{P}} \vee 0\right)}^{\infty} \sum_{\substack{\widetilde{Q} \in \mathcal{Q}\left(\mathbb{R}^{n-1}\right) \\
\ell(\widetilde{Q})=2^{-j}}} 2^{-j} \int_{\widetilde{Q}}\left[2^{j s(\widetilde{x}, 0)}\left|\lambda_{\widetilde{Q}}^{(i)}\right||\widetilde{Q}|^{-\frac{1}{2}} \frac{\chi_{\widetilde{P}}(\widetilde{x})}{C_{0} \widetilde{\phi}(\widetilde{P})}\right]^{p(\widetilde{x}, 0)} d \widetilde{x} \\
& \sim \sum_{j=\left(j_{\widetilde{P}} \vee 0\right)}^{\infty} \sum_{\substack{\widetilde{Q} \in \mathcal{Q}\left(\mathbb{R}^{n-1}\right) \\
\ell(\widetilde{Q})=2^{-j}}} \int_{\widetilde{\widetilde{Q}}_{i}}\left[2^{j s(\widetilde{x}, 0)} \frac{\left|\lambda_{\widetilde{Q}}^{(i)}\right|}{|\widetilde{Q}|^{\frac{1}{2}}} \frac{\chi_{\widetilde{P}}(\widetilde{x})}{C_{0} \widetilde{\phi}(\widetilde{P})}\right]^{p(\widetilde{x}, 0)} d \widetilde{x} d x_{n}
\end{aligned}
$$




$$
\begin{aligned}
& \lesssim \sum_{j=\left(j_{\widetilde{P}} \vee 0\right)}^{\infty} \int_{2(\widetilde{P} \times[0, \ell(\widetilde{P})))} \sum_{\substack{\widetilde{Q} \in \mathcal{Q}\left(\mathbb{R}^{n-1}\right) \\
\ell(\widetilde{Q})=2^{-j}}}\left[2^{j s(\widetilde{x}, 0)}\left|\lambda_{\widetilde{Q}}^{(i)}\right||\widetilde{Q}|^{-\frac{1}{2}}\right. \\
& \left.\quad \times\left[C_{0} \widetilde{\phi}(\widetilde{P})\right]^{-1} \chi_{\widetilde{\widetilde{Q}}_{i}}\left(\widetilde{x}, x_{n}\right)\right]^{p(\widetilde{x}, 0)} d \widetilde{x} d x_{n},
\end{aligned}
$$

where

$$
\widehat{\widetilde{Q}}_{i}:=\widetilde{Q} \times\left[\frac{(2 i-1) \ell(\widetilde{Q})}{2}, i \ell(\widetilde{Q})\right]
$$

which, combined with the fact that $\left\{\widehat{\widetilde{Q}}_{i}\right\}_{\widetilde{Q} \in \mathcal{Q}^{*}\left(\mathbb{R}^{n-1}\right)}$ are disjoint each other, (4.15) and the condition (S1) of $\phi$, implies that, for all $\widetilde{P} \in \mathcal{Q}\left(\mathbb{R}^{n-1}\right)$,

$$
\begin{aligned}
\mathrm{I}(\widetilde{P}) \lesssim \int_{2(\widetilde{P} \times[0, \ell(\widetilde{P}))}\left\{\sum _ { j = ( j _ { \widetilde { P } \vee 0 ) } } ^ { \infty } \left[\sum_{\substack{\widetilde{Q} \in \mathcal{Q}\left(\mathbb{R}^{n-1}\right) \\
\ell(\widetilde{Q})=2^{-j}}} 2^{j s(\widetilde{x}, 0)}\left|\lambda_{\widetilde{Q}}^{(i)}\right||\widetilde{Q}|^{-\frac{1}{2}}\right.\right. \\
\left.\left.\times \frac{1}{C_{0} \widetilde{\phi}(\widetilde{P})} \chi_{\widetilde{Q} \times\left[\frac{(2 i-1) \ell(\widetilde{Q})}{2}, i \ell(\widetilde{Q})\right)}\right]^{p(x)}\right\}^{\frac{p(\widetilde{x}, 0)}{p(x)}} d \widetilde{x} d x_{n} \\
\lesssim \int_{2(\widetilde{P} \times[0, \ell(\widetilde{P}))}\left[\sum _ { j = ( j _ { \widetilde { P } } \vee 0 ) } ^ { \infty } \left[\sum_{\substack{\widetilde{Q} \in \mathcal{Q}\left(\mathbb{R}^{n-1}\right) \\
\ell(\widetilde{Q})=2^{-j}}} 2^{j s(x)}\left|t_{\widetilde{Q} \times[(i-1) \ell(\widetilde{Q}), i \ell(\widetilde{Q}))}\right|\right.\right. \\
\left.\times \frac{|\ell(\widetilde{Q})|^{-\frac{n}{2}}}{C_{0} \phi(\widetilde{P} \times[0, \ell(\widetilde{P})))} \chi_{\widetilde{Q} \times\left[\frac{(2 i-1) \ell(\widetilde{Q})}{2}, i \ell(\widetilde{Q})\right)}^{p(x)}\right\} d x \lesssim 1,
\end{aligned}
$$

where we used the fact that $p(\widetilde{x}, 0)=p\left(\widetilde{x}, x_{n}\right)$ for all $\left(\widetilde{x}, x_{n}\right) \in \mathbb{R}^{n}$ with $\left|x_{n}\right| \leq 2$ in the last inequality. By this and Remark 1.1(ii), we conclude that, for all $\widetilde{P} \in \mathcal{Q}\left(\mathbb{R}^{n-1}\right)$,

$$
\frac{1}{\widetilde{\phi}(\widetilde{P})}\left\|\left\{\sum_{j=\left(j_{\widetilde{P}} \vee 0\right)}^{\infty}\left[\sum_{\substack{\widetilde{Q} \in \mathcal{Q}^{*}\left(\mathbb{R}^{n-1}\right) \\ \ell(\widetilde{Q})=2^{-j}}} 2^{j \beta(\cdot, 0)}\left|\lambda_{\widetilde{Q}}^{(i)}\right||\widetilde{Q}|^{-\frac{1}{2}} \chi_{\widetilde{Q}}\right]^{p(r, 0)}\right\}^{\frac{1}{p(\cdot, 0)}}\right\|_{L^{p(r, 0)}(\widetilde{P})}
$$

is finite, which implies that $\left\|\lambda^{(i)}\right\|_{f_{p(r, 0)}^{\beta(\cdot, 0), \widetilde{\phi}}\left(\mathbb{R}^{n-1}\right)} \lesssim 1$, namely, (4.14) holds true. Therefore,

$$
\|\operatorname{Tr}(f)\|_{F_{p(r, 0)}^{\beta(\cdot, 0), \tilde{\phi}}\left(\mathbb{R}^{n-1}\right)} \lesssim \sum_{i=0}^{2}\left\|\lambda^{(i)}\right\|_{f_{p(\cdot, 0)}^{\beta(r, 0), \tilde{\phi}}\left(\mathbb{R}^{n-1}\right)} \lesssim\|f\|_{F_{p(\cdot), p(\cdot)}^{s(\cdot), \phi}\left(\mathbb{R}^{n}\right)} .
$$


Step 2) We prove that the operator Tr is surjective. Let $f \in F_{p(\cdot, 0)}^{\beta(\cdot, 0), \widetilde{\phi}}\left(\mathbb{R}^{n-1}\right)$. Then, by Theorem 3.8, we find that there exist a sequence

$$
\lambda:=\left\{\lambda_{\widetilde{Q}}\right\}_{\widetilde{Q} \in \mathcal{Q}^{*}\left(\mathbb{R}^{n-1}\right)} \subset \mathbb{C}
$$

and a sequence $\left\{a_{\widetilde{Q}}\right\}_{\widetilde{Q} \in \mathcal{Q}^{*}\left(\mathbb{R}^{n-1}\right)}$ of $(K, L)$-smooth atoms of $F_{p(\cdot, 0)}^{\beta(\cdot, 0), \widetilde{\phi}}\left(\mathbb{R}^{n-1}\right)$ with $K$ and $L$ satisfying $(4.10)$ such that $f=\sum_{\widetilde{Q} \in \mathcal{Q}^{*}\left(\mathbb{R}^{n-1}\right)} \lambda_{\widetilde{Q}} a_{\widetilde{Q}}$ converges in $\mathcal{S}^{\prime}\left(\mathbb{R}^{n-1}\right)$ and

$$
\|\lambda\|_{f_{p(r, 0)}^{\beta(r, 0), \tilde{\phi}}\left(\mathbb{R}^{n-1}\right)} \lesssim\|f\|_{F_{p(r, 0)}^{\beta(r, 0), \tilde{\phi}}\left(\mathbb{R}^{n-1}\right)}
$$

moreover, for all $\widetilde{P} \in \mathcal{Q}\left(\mathbb{R}^{n-1}\right)$,

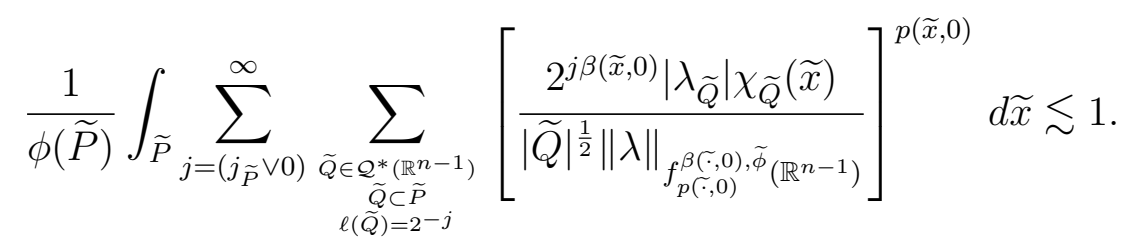

Let $\eta \in C_{c}^{\infty}(\mathbb{R})$ satisfy $\operatorname{supp} \eta \subset\left(-\frac{1}{2}, \frac{1}{2}\right)$ and $\eta(0)=1$. For all $\widetilde{Q} \in \mathcal{Q}\left(\mathbb{R}^{n-1}\right)$ and $\xi \in \mathbb{R}$, let $\eta_{\widetilde{Q}}(\xi):=\eta\left(2^{-\log _{2} \ell(\widetilde{Q})} \xi\right)$,

$$
g:=\sum_{\widetilde{Q} \in \mathcal{Q}^{*}\left(\mathbb{R}^{n-1}\right)} \lambda_{\widetilde{Q}} a_{\widetilde{Q}} \otimes \eta_{\widetilde{Q}}=: \sum_{Q \in \mathcal{Q}^{*}\left(\mathbb{R}^{n}\right)} t_{Q} b_{Q},
$$

where, for all $Q \in \mathcal{Q}^{*}\left(\mathbb{R}^{n}\right)$ and $x:=\left(\widetilde{x}, x_{n}\right) \in \mathbb{R}^{n}$,

$$
b_{Q}(x):=[\ell(\widetilde{Q})]^{-\frac{1}{2}} a_{\widetilde{Q}} \otimes \eta_{\widetilde{Q}}(x):=[\ell(\widetilde{Q})]^{-\frac{1}{2}} a_{\widetilde{Q}}(\widetilde{x}) \eta_{\widetilde{Q}}\left(x_{n}\right),
$$

$t_{Q}:=[\ell(\widetilde{Q})]^{1 / 2} \lambda_{\widetilde{Q}}$ if $Q=\widetilde{Q} \times[0, \ell(\widetilde{Q}))$ and $t_{Q}:=0$ otherwise.

Next we show that $g$ converges in $\mathcal{S}^{\prime}\left(\mathbb{R}^{n}\right)$ and

$$
\|g\|_{F_{p(\cdot), p(\cdot)}^{s(\cdot),}\left(\mathbb{R}^{n}\right)} \lesssim\|f\|_{F_{p(r, 0)}^{\beta(\cdot, 0), \widetilde{\phi}\left(\mathbb{R}^{n-1}\right)}} .
$$

It is easy to show that each $b_{Q}$ is a $(K, L)$-smooth atom supported near $Q$ of $F_{p(\cdot), p(\cdot)}^{s(\cdot),}\left(\mathbb{R}^{n}\right)$ with $K$ and $L$ as in (4.10). By Proposition 4.6 and the fact that $\left\{\widetilde{Q} \times\left[\frac{1}{2} \ell(\widetilde{Q}), \ell(\widetilde{Q})\right)\right\}_{\widetilde{Q} \in \mathcal{Q}^{*}\left(\mathbb{R}^{n-1}\right)}$ are disjoint each other, we find that

$$
\begin{aligned}
& \left\|\left\{t_{Q}\right\}_{Q \in \mathcal{Q}^{*}\left(\mathbb{R}^{n}\right)}\right\|_{f_{p(\cdot), p(\cdot)}^{\left.s(\cdot), \mathbb{R}^{n}\right)}} \\
& =\sup _{P \in \mathcal{Q}\left(\mathbb{R}^{n}\right)} \frac{1}{\phi(P)} \|\left\{\sum _ { j = ( j _ { P } \vee 0 ) } ^ { \infty } \left[\sum_{\widetilde{Q} \in \Theta_{j}} 2^{j s(\cdot)}\left|\lambda_{\widetilde{Q}}\right|[\ell(\widetilde{Q})]^{1 / 2}\right.\right. \\
& \left.\left.\quad \times|\widetilde{Q} \times[0, \ell(\widetilde{Q}))|^{-1 / 2} \chi_{\widetilde{Q} \times[0, \ell(\widetilde{Q}))}\right]^{p(\cdot)}\right\}^{1 / p(\cdot)}\left\|_{L^{p(\cdot)(P)}}\right\|\left\|_{P \in \mathcal{Q}\left(\mathbb{R}^{n}\right)} \frac{1}{\phi(P)}\right\| \sum_{j=\left(j_{P} \vee 0\right)}^{\infty} \sum_{\widetilde{Q} \in \Theta_{j}} 2^{j s(\cdot)}\left|\lambda_{\widetilde{Q}}\right||\widetilde{Q}|^{-\frac{1}{2}} \chi_{\widetilde{Q} \times\left[\frac{1}{2} \ell(\widetilde{Q}), \ell(\widetilde{Q})\right)} \|_{L^{p(\cdot)(P)}}
\end{aligned}
$$




$$
\begin{aligned}
& \sim \sup _{\widetilde{P} \in \mathcal{Q}\left(\mathbb{R}^{n-1}\right)} \frac{1}{\widetilde{\phi}(\widetilde{P})} \| \sum_{\substack{\widetilde{Q} \in \mathcal{Q}^{*}\left(\mathbb{R}^{n-1}\right) \\
\widetilde{Q} \subset \widetilde{P}}}|\widetilde{Q}|^{-\frac{s(\cdot)}{n-1}}\left|\lambda_{\widetilde{Q}}\right| \\
& \quad \times|\widetilde{Q}|^{-1 / 2} \chi_{\widetilde{Q} \times\left[\frac{1}{2} \ell(\widetilde{Q}), \ell(\widetilde{Q})\right)} \|_{L^{p(\cdot)}(\widetilde{P} \times[0, \ell(\widetilde{P})))},
\end{aligned}
$$

where $\Theta_{j}:=\left\{\widetilde{Q} \in \mathcal{Q}^{*}\left(\mathbb{R}^{n-1}\right): \widetilde{Q} \times[0, \ell(\widetilde{Q})) \subset P, \ell(\widetilde{Q})=2^{-j}\right\}$. On the other hand, let $\Gamma:=\|\lambda\|_{f_{p(r, 0)}^{\beta(,, 0), \widetilde{\phi}}\left(\mathbb{R}^{n-1}\right)}$. Then, for all $\widetilde{P} \in \mathcal{Q}\left(\mathbb{R}^{n-1}\right)$, by (4.17), we find that

$$
\begin{aligned}
& \frac{1}{\widetilde{\phi}(\widetilde{P})} \int_{\widetilde{P} \times[0, \ell(\widetilde{P}))}\left\{\sum_{\substack{\widetilde{Q} \in \mathcal{Q}^{*}\left(\mathbb{R}^{n-1}\right) \\
\widetilde{Q} \subset \widetilde{P}}}\left|\lambda_{\widetilde{Q}}\right||\widetilde{Q}|^{-\frac{1}{2}} \Gamma^{-1}|\widetilde{Q}|^{-\frac{s(x)}{n-1}} \chi_{\widetilde{Q} \times\left[\frac{1}{2} \ell(\widetilde{Q}), \ell(\widetilde{Q})\right)}\right\}^{p(x)} d x \\
& \sim \frac{1}{\widetilde{\phi}(\widetilde{P})} \sum_{\substack{\widetilde{Q} \in \mathcal{Q}^{*}\left(\mathbb{R}^{n-1}\right) \\
\widetilde{Q} \subset \widetilde{P}}} \int_{\widetilde{Q} \times\left[\frac{1}{2} \ell(\widetilde{Q}), \ell(\widetilde{Q})\right)}\left[|\widetilde{Q}|^{-\frac{s(x)}{n-1}-\frac{1}{2}}\left|\lambda_{\widetilde{Q}}\right| \Gamma^{-1}\right]^{p(x)} d x \\
& \sim \frac{1}{\widetilde{\phi}(\widetilde{P})} \sum_{j=\left(j_{\widetilde{P} \vee 0)}\right.}^{\infty} \sum_{\substack{\widetilde{Q} \in \mathcal{Q}^{*}\left(\mathbb{R}^{n-1}\right) \\
\widetilde{Q} \subset \widetilde{P} \\
\ell(\widetilde{Q})=2^{-j}}} \int_{\widetilde{Q}}\left[2^{j \beta(\widetilde{x}, 0)}\left|\lambda_{\widetilde{Q}}\right||\widetilde{Q}|^{-\frac{1}{2}} \Gamma^{-1}\right]^{p(\widetilde{x}, 0)} d \widetilde{x} \lesssim 1,
\end{aligned}
$$

which, together with Remark 1.1(ii), implies that

$$
\begin{aligned}
& \frac{1}{\widetilde{\phi}(\widetilde{P})}\left\|\sum_{\substack{\widetilde{Q} \in \mathcal{Q}^{*}\left(\mathbb{R}^{n-1}\right) \\
\widetilde{Q} \subset \widetilde{P}}}|\widetilde{Q}|^{-\frac{s(\cdot)}{n-1}}\left|\lambda_{\widetilde{Q}}\right||\widetilde{Q}|^{-\frac{1}{2}} \chi_{\widetilde{Q} \times\left[\frac{1}{2} \ell(\widetilde{Q}), \ell(\widetilde{Q})\right)}\right\|_{\substack{L^{p(\cdot)}(\widetilde{P} \times[0, \ell(\widetilde{P})))\\
}} \\
& \lesssim\|\lambda\|_{f_{p(\ulcorner, 0)}^{\beta(\cdot, 0), \widetilde{\phi}}\left(\mathbb{R}^{n-1}\right)} .
\end{aligned}
$$

From this and (4.18), we further deduce that

$$
\left\|\left\{t_{Q}\right\}_{Q \in \mathcal{Q}^{*}\left(\mathbb{R}^{n}\right)}\right\|_{f_{p(\cdot), p(\cdot)}^{s(\cdot),}\left(\mathbb{R}^{n}\right)} \lesssim\|\lambda\|_{f_{p(r, 0)}^{\beta(\cdot, 0), \widetilde{\phi}}\left(\mathbb{R}^{n-1}\right)}
$$

Therefore, by Theorem 3.8(i) and (4.16), we conclude that $g=\sum_{Q \in \mathcal{Q}^{*}\left(\mathbb{R}^{n}\right)} t_{Q} b_{Q}$ converges in $\mathcal{S}^{\prime}\left(\mathbb{R}^{n}\right), g \in F_{p(\cdot), p(\cdot)}^{s(\cdot),}\left(\mathbb{R}^{n}\right)$ and

$$
\|g\|_{F_{p(\cdot), p(\cdot)}^{s(\cdot),}\left(\mathbb{R}^{n}\right)} \lesssim\|f\|_{F_{p(\cdot, 0)}^{\beta(\cdot, 0), \widetilde{\phi}}\left(\mathbb{R}^{n-1}\right)} ;
$$

furthermore, $\operatorname{Tr}(g)=f$ in $\mathcal{S}^{\prime}\left(\mathbb{R}^{n-1}\right)$, which implies that

$$
\operatorname{Tr}: F_{p(\cdot), p(\cdot)}^{s(\cdot),}\left(\mathbb{R}^{n}\right) \rightarrow F_{p(\cdot, 0), p(\ulcorner, 0)}^{s(\ulcorner, 0), \widetilde{\phi}}\left(\mathbb{R}^{n-1}\right)
$$

is surjective and hence completes the proof of Theorem 4.1. 
Acknowledgement. The authors would like to express their deep thanks to referees for their careful reading and many useful comments which improve the presentation of this article. This project is supported by the National Natural Science Foundation of China (Grant Nos. 11171027, 11361020 and 11471042), the Specialized Research Fund for the Doctoral Program of Higher Education of China (Grant No. 20120003110003) and the Fundamental Research Funds for Central Universities of China (Grant Nos. 2012LYB26, 2012CXQT09, 2013YB60 and 2014KJJCA10).

\section{REFERENCES}

1. E. Acerbi and G. Mingione, Regularity results for stationary electro-rheological fluids, Arch. Ration. Mech. Anal. 164 (2002), 213-259.

2. E. Acerbi and G. Mingione, Regularity results for electrorheological fluids: the stationary case, C. R. Math. Acad. Sci. Paris 334 (2002), 817-822.

3. E. Acerbi and G. Mingione, Gradient estimates for the $p(x)$-Laplacean system, J. Reine Angew. Math. 584 (2005), 117-148.

4. A. Almeida and P. Hästö, Besov spaces with variable smoothness and integrability, J. Funct. Anal. 258 (2010), 1628-1655.

5. A. Almeida and S. Samko, Characterization of Riesz and Bessel potentials on variable Lebesgue spaces, J. Funct. Spaces Appl. 4 (2006), 113-144.

6. K. Astala, T. Iwaniec, P. Koskela and G. Martin, Mappings of BMO-bounded distortion, Math. Ann. 317 (2000), 703-726.

7. O. Besov, On spaces of functions of variable smoothness defined by pseudodifferential operators, Tr. Mat. Inst. Steklova 227 (1999)(in Russian), Issled. po Teor. Differ. Funkts. Mnogikh Perem. i ee Prilozh. 18, 56-74, translation in: Proc. Steklov Inst. Math. 227 (1999), 50-69.

8. O. Besov, Equivalent normings of spaces of functions of variable smoothness, Tr. Mat. Inst. Steklova 243 (2003) (in Russian), Funkts. Prostran., Priblizh., Differ. Uravn., 87-95, translation in: Proc. Steklov Inst. Math. 243 (2003), 80-88.

9. O. Besov, Interpolation, embedding, and extension of spaces of functions of variable smoothness, Tr. Mat. Inst. Steklova 248 (2005) (in Russian), Issled. po Teor. Funkts. i Differ. Uravn., 52-63, translation in: Proc. Steklov Inst. Math. 248 (2005), 47-58.

10. Z. Birnbaum and W. Orlicz, Über die Verallgemeinerung des Begriffes der zueinander konjugierten Potenzen, Studia Math. 3 (1931), 1-67.

11. Y. Chen, S. Levine and M. Rao, Variable exponent, linear growth functionals in image restoration, SIAM J. Appl. Math. 66 (2006), 1383-1406.

12. D. Cruz-Uribe, L. Diening and P. Hästö, The maximal operator on weighted variable Lebesgue spaces, Fract. Calc. Appl. Anal. 14 (2011), 361-374.

13. D. Cruz-Uribe and A. Fiorenza, Variable Lebesgue Spaces, Foundations and Harmonic Analysis, in: Applied and Numerical Harmonic Analysis, Birkhäuser/Springer, Heidelberg, 2013.

14. D. Cruz-Uribe, A. Fiorenza, J. M. Martell and C. Pérez, The boundedness of classical operators on variable $L^{p}$ spaces, Ann. Acad. Sci. Fenn. Math. 31 (2006), 239-264.

15. D. Cruz-Uribe and L.-A. D. Wang, Variable Hardy spaces, Indiana Univ. Math. J. 63 (2014), 447-493.

16. G. Dafni and J. Xiao, Some new tent spaces and duality theorems for fractional Carleson measures and $Q_{\alpha}\left(\mathbb{R}^{n}\right)$, J. Funct. Anal. 208 (2004), 377-422.

17. L. Diening, Maximal function on generalized Lebesgue spaces $L^{p(\cdot)}\left(\mathbb{R}^{n}\right)$, Math. Inequal. Appl. 7 (2004), 245-253. 
18. L. Diening, P. Harjulehto, P. Hästö, Y. Mizuta and T. Shimomura, Maximal functions in variable exponent spaces: limiting cases of the exponent, Ann. Acad. Sci. Fenn. Math. 34 (2009), 503-522.

19. L. Diening, P. Harjulehto, P. Hästö and M. Růžička, Lebesgue and Sobolev Spaces with Variable Exponents, Lecture Notes in Mathematics 2017, Springer, Heidelberg, 2011.

20. L. Diening, P. Hästö and S. Roudenko, Function spaces of variable smoothness and integrability, J. Funct. Anal. 256 (2009), 1731-1768.

21. L. Diening and M. Růžička, Calderón-Zygmund operators on generalized Lebesgue spaces $L^{p(\cdot)}$ and problems related to fluid dynamics, J. Reine Angew. Math. 563 (2003), 197-220.

22. D. Drihem, Atomic decomposition of Besov spaces with variable smoothness and integrability, J. Math. Anal. Appl. 389 (2012), 15-31.

23. D. Drihem, Atomic decomposition of Besov-type and Triebel-Lizorkin-type spaces, Sci. China Math. 56 (2013), 1073-1086.

24. M. Essén, S. Janson, L. Z. Peng and J. Xiao, Q spaces of several real variables, Indiana Univ. Math. J. 49 (2000), 575-615.

25. X. L. Fan, Global $C^{1, \alpha}$ regularity for variable exponent elliptic equations in divergence form, J. Differential Equations 235 (2007), 397-417.

26. M. Frazier and B. Jawerth, Decomposition of Besov spaces, Indiana Univ. Math. J. 34 (1985), 777-799.

27. M. Frazier and B. Jawerth, A discrete transform and decompositions of distribution spaces, J. Funct. Anal. 93 (1990), 34-170.

28. M. Frazier, B. Jawerth and G. Weiss, Littlewood-Paley Theory and the Study of Function Spaces, CBMS Regional Conference Series in Mathematics, 79, Published for the Conference Board of the Mathematical Sciences, Washington, DC; by the American Mathematical Society, Providence, RI, 1991.

29. H.F. Gonçalves, S.D. Moura and J.S. Neves, On trace spaces of 2-microlocal type spaces, J. Funct. Anal. 267 (2014), 3444-3468.

30. P. Gurka, P. Harjulehto and A. Nekvinda, Bessel potential spaces with variable exponent, Math. Inequal. Appl. 10 (2007), 661-676.

31. K.-P. Ho, Vector-valued singular integral operators on Morrey type spaces and variable Triebel-Lizorkin-Morrey spaces, Ann. Acad. Sci. Fenn. Math. 37 (2012), 375-406.

32. T. Iwaniec and J. Onninen, $H^{1}$-estimates of Jacobians by subdeterminants, Math. Ann. 324 (2002), 341-358.

33. M. Izuki, E. Nakai and Y. Sawano, Function spaces with variable exponents - an introduction, Sci. Math. Jpn. 77 (2014), 187-315.

34. H. Kempka, 2-microlocal Besov and Triebel-Lizorkin spaces of variable integrability, Rev. Mat. Complut. 22 (2009), 227-251.

35. H. Kempka, Atomic, molecular and wavelet decomposition of 2-microlocal Besov and Triebel-Lizorkin spaces with variable integrability, Funct. Approx. Comment. Math. 43 (2010), part 2, 171-208.

36. H. Kempka and J. Vybíral, Spaces of variable smoothness and integrability: characterizations by local means and ball means of differences, J. Fourier Anal. Appl. 18 (2012), 852-891.

37. O. Kováčik and J. Rákosník, On spaces $L^{p(x)}$ and $W^{k, p(x)}$, Czechoslovak Math. J. 41 (116) (1991), 592-618.

38. H.-G. Leopold, On Besov spaces of variable order of differentiation, Z. Anal. Anwendungen 8 (1989), 69-82.

39. H.-G. Leopold, Interpolation of Besov spaces of variable order of differentiation, Arch. Math. (Basel) 53 (1989), 178-187.

40. H.-G. Leopold, On function spaces of variable order of differentiation, Forum Math. 3 (1991), 1-21.

41. H.-G. Leopold, Embedding of function spaces of variable order of differentiation in function spaces of variable order of integration, Czechoslovak Math. J. 49(124) (1999), 633-644. 
42. H.-G. Leopold and E. Schrohe, Trace theorems for Sobolev spaces of variable order of differentiation, Math. Nachr. 179 (1996), 223-245.

43. P. Li, J. Xiao and Q. Yang, Global mild solutions to modified Navier-Stokes equations with small initial data in critical Besov-Q spaces, Electron. J. Differential Equations 2014, No. 185, 37 pp.

44. P. Li and Z. Zhai, Generalized Navier-Stokes equations with initial data in local Q-type spaces, J. Math. Anal. Appl. 369 (2010), 595-609.

45. P. Li and Z. Zhai, Well-posedness and regularity of generalized Navier-Stokes equations in some critical Q-spaces, J. Funct. Anal. 259 (2010), 2457-2519.

46. Y. Liang, Y. Sawano, T. Ullrich, D. Yang and W. Yuan, New characterizations of BesovTriebel-Lizorkin-Hausdorff spaces including coorbits and wavelets, J. Fourier Anal. Appl. 18 (2012), 1067-1111.

47. Y. Liang, D. Yang, W. Yuan, Y. Sawano and T. Ullich, A new framework for generalized Besov-type and Triebel-Lizorkin-type spaces, Dissertationes Math. (Rozprawy Mat.) 489 (2013), 1-114.

48. S. Martínez and N. Wolanski, A minimum problem with free boundary in Orlicz spaces, Adv. Math. 218 (2008), 1914-1971.

49. S.D. Moura, J.S. Neves and C. Schneider, On trace spaces of 2-microlocal Besov spaces with variable integrability, Math. Nachr. 286 (2013), 1240-1254.

50. E. Nakai, Pointwise multipliers for functions of weighted bounded mean oscillation, Studia Math. 105 (1993), 105-119.

51. E. Nakai, The Campanato, Morrey and Hölder spaces on spaces of homogeneous type, Studia Math. 176 (2006), 1-19.

52. E. Nakai, Generalized fractional integrals on generalized Morrey spaces, Math. Nachr. 287 (2014), 339-351.

53. E. Nakai and Y. Sawano, Hardy spaces with variable exponents and generalized Campanato spaces, J. Funct. Anal. 262 (2012), 3665-3748.

54. H. Nakano, Modulared Semi-Ordered Linear Spaces, Maruzen Co., Ltd., Tokyo, 1950.

55. H. Nakano, Topology of Linear Topological Spaces, Maruzen Co., Ltd., Tokyo, 1951.

56. T. Noi, Trace and extension operators for Besov spaces and Triebel-Lizorkin spaces with variable exponents, arXiv: 1410.1185.

57. T. Noi and Y. Sawano, Complex interpolation of Besov spaces and Triebel-Lizorkin spaces with variable exponents, J. Math. Anal. Appl. 387 (2012), 676-690.

58. W. Orlicz, Über eine gewisse Klasse von Räumen vom Typus B, Bull. Int. Acad. Pol. Ser. A 8 (1932), 207-220.

59. M. Růžička, Electrorheological fluids: modeling and mathematical theory, Lecture Notes in Mathematics, 1748, Springer-Verlag, Berlin, 2000.

60. M. Růžička, Modeling, mathematical and numerical analysis of electrorheological fluids, Appl. Math. 49 (2004), 565-609.

61. Y. Sawano, Wavelet characterization of Besov-Morrey and Triebel-Lizorkin-Morrey spaces, Funct. Approx. Comment. Math. 38 (2008), 93-107.

62. Y. Sawano and H. Tanaka, Decompositions of Besov-Morrey spaces and Triebel-LizorkinMorrey spaces, Math. Z. 257 (2007), 871-905.

63. W. Sickel, Smoothness spaces related to Morrey spaces-a survey. I, Eurasian Math. J. 3 (2012), 110-149.

64. W. Sickel, Smoothness spaces related to Morrey spaces-a survey. II, Eurasian Math. J. 4 (2013), 82-124.

65. E. M. Stein, Harmonic Analysis: Real-Variable Methods, Orthogonality, and Oscillatory Integrals, Princeton Univ. Press, Princeton, N.J., 1993.

66. H. Triebel, Theory of Function Spaces, Birkhäuser Verlag, Basel, 1983.

67. H. Triebel, Theory of Function Spaces II, Birkhäuser Verlag, Basel, 1992.

68. H. Triebel, Interpolation Theory, Function Spaces, Differential Operators, Second edition, Johann Ambrosius Barth, Heidelberg, 1995. 
69. H. Triebel, Theory of Function Spaces III, Birkhäuser Verlag, Basel, 2006.

70. H. Triebel, Local Function Spaces, Heat and Navier-Stokes Equations, EMS Tracts in Mathematics 20, European Mathematical Society (EMS), Zürich, 2013.

71. T. Ullrich, Continuous characterizations of Besov-Lizorkin-Triebel spaces and new interpretations as coorbits, J. Funct. Spaces Appl. 2012, Art. ID 163213, 47 pp.

72. J. Vybíral, Sobolev and Jawerth embeddings for spaces with variable smoothness and integrability, Ann. Acad. Sci. Fenn. Math. 34 (2009), 529-544.

73. J. Xu, Variable Besov and Triebel-Lizorkin spaces, Ann. Acad. Sci. Fenn. Math. 33 (2008), $511-522$.

74. J. Xu, The relation between variable Bessel potential spaces and Triebel-Lizorkin spaces, Integral Transforms Spec. Funct. 19 (2008), 599-605.

75. J. Xu, An atomic decomposition of variable Besov and Triebel-Lizorkin spaces, Armen. J. Math. 2 (2009), 1-12.

76. D. Yang and W. Yuan, A new class of function spaces connecting Triebel-Lizorkin spaces and $Q$ spaces, J. Funct. Anal. 255 (2008), 2760-2809.

77. D. Yang and W. Yuan, New Besov-type spaces and Triebel-Lizorkin-type spaces including Q spaces, Math. Z. 265 (2010), 451-480.

78. D. Yang and W. Yuan, Relations among Besov-type spaces, Triebel-Lizorkin-type spaces and generalized Carleson measure spaces, Appl. Anal. 92 (2013), 549-561.

79. D. Yang, W. Yuan and C. Zhuo, Complex interpolation on Besov-type and Triebel-Lizorkintype spaces, Anal. Appl. (Singap.) 11 (2013), 1350021, 45 pp.

80. W. Yuan, W. Sickel and D. Yang, Morrey and Campanato Meet Besov, Lizorkin and Triebel, Lecture Notes in Mathematics 2005, Springer-Verlag, Berlin, 2010.

81. W. Yuan, W. Sickel and D. Yang, On the coincidence of certain approaches to smoothness spaces related to Morrey spaces, Math. Nachr. 286 (2013), 1571-1584.

82. C. Zhuo, D. Yang and Y. Liang, Intrinsic square function characterizations of Hardy spaces with variable exponents, Bull. Malays. Math. Sci. Soc. (2), to appear.

School of Mathematical Sciences, Beijing Normal University, Laboratory of Mathematics and Complex Systems, Ministry of Education, Beijing 100875, PeoPLE'S REPUBlic of China.

E-mail address: dcyang@bnu.edu.cn

E-mail address: cqzhuo@mail.bnu.edu.cn

E-mail address: wenyuan@bnu.edu.cn 